To appear: Proceedings of the London Mathematical Society

May 16th, 1995

\title{
Dimensions and measures in infinite iterated function systems
}

\author{
R. Daniel Mauldin ${ }^{1}$ and Mariusz Urbański ${ }^{1,2}$ \\ Department of Mathematics, University of North Texas, \\ Denton TX 76203-5116, USA
}

\begin{abstract}
The Hausdorff and packing measures and dimensions of the limit sets of iterated function systems generated by countable families of conformal contractions are investigated. Conformal measures for such systems, reflecting geometric properties of the limit set, are introduced, proven to exist, and to be unique. The existence of a unique invariant probability equivalent to the conformal measure is derived. Our methods employ the concepts of the Perron-Frobenius operator, symbolic dynamics on an infinite dimensional shift space, and the properties of the above mentioned ergodic invariant measure. A formula for the Hausdorff dimension of the limit set in terms of the pressure function is derived. Fractal phenomena not exhibited by finite systems are shown to appear in the infinite case. In particular a variety of conditions are provided for Hausdorff and packing measures to be positive or finite, and a number of examples are described showing the appearance of various possible combinations for these quantities. One example given special attention is the limit set associated to the complex continued fraction expansion - in particular lower and upper estimates for its Hausdorff dimension are given. A large natural class of systems whose limit sets are "dimensionless in the restricted sense" is described.
\end{abstract}

\footnotetext{
${ }^{1}$ Research supported by NSF Grant DMS 9303888

${ }^{2}$ Research supported by KBN Grant 211109101

1991 Mathematics Subject Classification: primary 28A80; secondary 58F08, 58F11, 28A78.
} 


\section{Introduction}

The concept of an iterated function system arises in two natural contexts. One is as a generalization of the process of looking at backward trajectories of a continuous map of a metric space. The second is as a geometric recursion generating a fractal set. It has turned out to be of importance for describing complexity of objects arising in systems directly modeling physical processes.

With any finite iterated function system is associated its limit set $J$, the fractal coded by this system. The qualitative metric structure of this set, expressed mainly as the equality of the three basic dimensions (Hausdorff, box, and packing) has been clarified beginning with the works of Moran [18] and Hutchinson [14] assuming that the generating mappings of the underlying systems are similarities. One can infer from [14] that the Hausdorff and packing measures coincide up to a multiplicative constant, and are positive and finite. More subtle achievements, focused for example around the problem of multifractal decomposition or around the meaning of several separation conditions have been then obtained (see $[28]$ ).

In the meantime a need to explore iterated function systems with an infinite set of generators consisting of conformal maps rather than simply similarities has arisen from both of these contexts (see [1], [5], [11], and [17] for example).

The main aim of this paper is to provide methods appropriate to deal with the case when the number of generators is not assumed to be finite nor the generators are required to be linear - merely conformal. After defining the limit set $J$, we then prove that an analog of the Moran-Bowen formula, identifying its Hausdorff dimension as the zero of the pressure function, continues to hold in our case in a slightly modified form. Namely, instead of the zero of the pressure function $\mathrm{P}(t)$, we take the infimum of all arguments $t>0$ for which $\mathrm{P}(t)$ is negative. This modification is important and in Section 5 examples are given (cf. Ex.5.3 and 5.4) of systems such that $\mathrm{P}(t)$ is always either infinite or negative (the phenomenon, first observed in [17], cannot happen in the finite case) and in this case, the corresponding limit sets are "dimensionless in the restricted sense"; there is no Hausdorff gauge function of the form $g(t)=t^{\alpha} L(t)$, where $L$ is slowing varying such that the corresponding Hausdorff measure gives J positive finite measure. The property that $\mathrm{P}(t)$ has some finite nonnegative value turns out to be a necessary and sufficient condition for the existence of a semiconformal measure, that is a fixed point of the associated PerronFrobenius operator. (Indeed, the iteration of infinitely many similarity maps, $\phi_{i}$ with reduction ratios $r_{i}$ is a special deterministic case of [17]. The results of [17] show that there is a $t$-conformal measure $m$ or self-similar measure $m$ satisfying $m=\sum_{i=1}^{\infty} r_{i}^{t} m \circ \phi_{i}^{-1}$ if and only if $\sum_{i=1}^{\infty} r_{i}^{t}=1$.) The semiconformal measure is then proved to be conformal which is the notion which links dynamical and geometrical features of a conformal iterated function system and its behaviour governs the geometric measure theoretic properties of the limit set. If it exists, the conformal measure is proven to be unique. We should also mention that although semiconformality is merely an auxiliary notion, it can be defined even if no separation condition is imposed, and in a number of our proofs the semiconformality property is sufficient.

We would like to remark at this point that the concept of conformal measure was first introduced by S. Patterson in [21] for limit sets of Fuchsian groups and then extended 
by D. Sullivan (see [32] and [33]) to the class of all Kleinian groups (i.e. discrete groups of isometries of a hyperbolic space of arbitrary dimension) and rational functions of the Riemann sphere. These three classes of systems, Kleinian groups, rational functions, and conformal iterated function systems, although similar, are mutually distinct. Let us list just a few prominent features differentiating them. Indeed, for both Kleinian groups (not necessarily geometrically finite) and rational functions a $t$-conformal measure always exists (see again [32] and [33]) whereas, as explained above, for conformal iterated function systems this property may fail. Also, for a conformal iterated function system if a $t$ conformal measure exists, then $t$ is equal to the Hausdorff dimension of the corresponding limit set and this measure is unique whereas for both Kleinian groups and rational functions there are known examples (see [19], [22], [4]) allowing parameters $t$ greater than Hausdorff dimension admitting conformal measures.

There are however wide subclasses of Kleinian groups and rational functions almost satisfying the requirements of conformal iterated function systems, so called convex cocompact Kleinian groups and hyperbolic rational functions. Hyperbolic rational functions admit finite Markov partitions with exponentially contracting "inverse branches"; convex cocompact Fuchsian groups, with an appropriate choice of generators, do the same, and all convex cocompact Kleinian groups are by some experts believed to admit such partitions too. A little problem appears here that the images of the elements of Markov partitions may not be equal the whole limit set but the definition of iterated function systems could be easily extended, for the price of some bigger technical complexity, to cover these cases.

On the other hand, the phenomenon of critical points, substantially complicating the behaviour of rational functions does not seem to have any reasonable analog in the class of Kleinian groups and conformal iterated function systems. However between the class of hyperbolic rational functions and those with critical points in the Julia sets (the common name for the limit set associated with a rational function) there is a class of rational functions which are not hyperbolic but do not allow any critical point in the Julia set. These maps, called parabolic maps, do not fit into formalism of conformal iterated function systems but one can associate to any of these maps a so called jump map (see [1], [5]), originally considered by Schweiger in [29] in the context of maps of an interval, which like hyperbolic maps, admits a Markov partition but consisting of infinitely many elements. This construction along with the complex continued fraction expansion system was in fact our primary clue leading us toward infinite iterated function systems.

Coming back to iterated function systems, in order to emphasize the difference between the finite and infinite case we would like to point out that even if a conformal measure exists, the Hausdorff measure may vanish, the packing measure may be infinite and the packing dimension (so also box dimension) can be larger than the Hausdorff dimension.

\section{Organization of paper}

In Section 2 we formalize our notation and setting and also make some preliminary observations. In Section 3 we introduce topological pressure, Perron-Frobenius operator, and conformal and semiconformal measures. We define and study here the class of regular and hereditarily regular systems, and using a symbolic representation of the limit set we prove the existence and uniqueness of the conformal measure. We also derive the existence 
of an invariant probability equivalent to the conformal measure. Proving its ergodicity we simultaneously show its uniqueness. At the end of the section we give a proof that if the system has finite entropy, then the Hausdorff dimension of the conformal measure is equal to the Hausdorff dimension of the limit set.

The next section, Section 4, is partially motivated by what happens in the context of Kleinian groups with cusps and parabolic rational functions (see [6], [34]). It is also partially motivated from the geometric recursion point of view for similarities. Even in the random case (see [12], [17]) there is a natural random measure on the random object. This measure is the random "conformal" measure and for these systems the Hausdorff measure is finite.

We prove in this section that as in the case of Kleinian groups and parabolic rational functions (even more, rational functions with no reccurent critical points in the Julia set (see [36])) Hausdorff measure is always finite and packing measure is positive. Moreover, we provide sufficient conditions for Hausdorff measure to vanish or to be positive and for packing measure to be infinite or finite. These conditions are formulated in terms of the boundary behaviour of our system and then in examples we show that all possibly allowed combinations are realized. A similar situation has been observed for Kleinian groups and parabolic rational functions (see [34], [6], and [36] for example) except that in this latter case at least one, Hausdorff or packing measure is always positive and finite. Notice also that in view of Theorem 4.17 if Hausdorff or packing measure is positive and finite, then, up to a multiplicative constant, it is equal to the conformal measure. Therefore the results of this section can be also viewed as an attempt to understand the geometric nature of conformal measures. And this process is not finished yet. Since, although we would be able to extract a fairly large class of gauge functions for which associated Hausdorff and packing measures either vanish or are infinite, we are not able to provide any example of a system admitting a conformal measure whose limit set would be "totally dimensionless"; there is no Hausdorff gauge function such that the corresponding Hausdorff measure gives the limit set positive finite measure.

We also prove in this section an exponential decay of the Lebesgue measures of consecutive "levels" of our systems and provide an effective sufficient condition for the Hausdorff dimension of the limit set to be stricly smaller than the dimension of the Euclidean space containing it. At the end of the section we prove that the limit sets of irregular systems are dimensionless.

Section 5 consists of several examples illustrating a wide variety of possible fractal behaviour of limits sets. In particular Example 5.2 gives evidence of how large the difference can be between limit sets of infinite systems and finite systems and as well between limit sets of infinite iterated function systems, limit sets of geometrically finite Kleinian groups and Julia sets of parabolic rational functions, as in the two last cases the Hausdorff dimension and box dimension are always equal (see [7] and [31]) whereas for iterated function systems even packing dimension can be bigger than Hausdorff dimension.

In Section 6, we deal with our primary example - an iterated function system associated to complex continued fraction expansions. From the results obtained in Sections 3 and 4 we conclude that the Hausdorff dimension of corresponding limit set lies strictly between 1 and 2, obtaining in this way a more qualitative proof than that given in [11]. 
We improve the quantitative approach worked out in [11] by using some properties of the pressure function. Enjoying the power of modern computers, we show that this dimension lies between the numbers 1.2484 and 1.9.

In Section 7, we gather together several problems which remain unsolved. In the appendix, we give a direct derivation of the ergodic probability measure equivalent to the conformal measure. This derivation differs from the method used in Section 3 in that no use of an abstract symbol space. Also, the measure is obtained in a constructive manner as compared to the derivation given in Section 3 which uses Banach limits.

\section{Preliminaries}

Throughout the whole paper the symbols $\lambda_{d}, V_{d}=\lambda_{d}(B(0,1))$, and $\lambda_{d-1}$ are reserved to denote respectively the $d$-dimensional Lebesgue measure on $\mathbb{R}^{d}$, the volume of the unit ball $B(0,1) \subset \mathbb{R}^{d}$, and the $(d-1)$-dimensional Lebesgue measure on the unit sphere $S^{d-1} \subset \mathbb{R}^{d}$.

Let $(X, \rho)$ be a nonempty compact metric space, let $I$ be a countable set with at least two elements, and let $S=\left\{\phi_{i}: X \rightarrow X: i \in I\right\}$ be a collection of injective contractions from $X$ to $X$ for which there exists $0<s<1$ such that

$$
\rho\left(\phi_{i}(x), \phi_{i}(y)\right) \leq s \rho(x, y),
$$

for every $i \in I$ and for every pair of points $x, y \in X$. Any such collection $S$ of contractions is called an iterated function system, frequently abbreviated as i.f.s. Put $I^{*}=\bigcup_{n \geq 1} I^{n}$ and for $\omega \in I^{n}, n \geq 1$, set

$$
\phi_{\omega}=\phi_{\omega_{1}} \circ \phi_{\omega_{2}} \circ \ldots \circ \phi_{\omega_{n}} .
$$

If $\omega \in I^{*} \cup I^{\infty}$ and $n \geq 1$ does not exceed the length of $\omega$, we denote by $\left.\omega\right|_{n}$ the word $\omega_{1} \omega_{2} \ldots \omega_{n}$. Observe now that given $\omega \in I^{\infty}$, the compact sets $\phi_{\left.\omega\right|_{n}}(X), n \geq 1$, are decreasing and their diameters converge to zero. In fact, by (2.1)

$$
\operatorname{diam}\left(\phi_{\left.\omega\right|_{n}}(X)\right) \leq s^{n} \operatorname{diam}(X)
$$

This implies that the set

$$
\pi(\omega)=\bigcap_{n=0}^{\infty} \phi_{\left.\omega\right|_{n}}(X)
$$

is a singleton and therefore this formula defines a map $\pi: I^{\infty} \rightarrow X$ which, in view of (2.2) is continuous. Let $\sigma: I^{\infty} \rightarrow I^{\infty}$ denote the left shift map (cutting out the first coordinate) on $I^{\infty}$, that is $\sigma(\omega)=\omega_{2} \omega_{3} \ldots$. We will frequently use the following obvious relation

$$
\pi \circ \sigma(\omega)=\phi_{\omega_{1}}^{-1} \circ \pi(\omega)
$$

The main object of our interest will be the set $J=\pi\left(I^{\infty}\right)=\bigcup_{\omega \in I^{\infty}} \bigcap_{n=1}^{\infty} \phi_{\omega \mid n}(X)$, called the limit set associated to the system $S=\left\{\phi_{i}: X \rightarrow X: i \in I\right\}$. Since $\phi_{i}(\pi(\omega))=\pi(i \omega)$ for every $i \in I$ and rewriting (2.3) in the form $\pi(\omega)=\phi_{\omega_{1}}(\pi(\sigma(\omega)))$, we see that

$$
J=\bigcup_{i \in I} \phi_{i}(J)
$$


Notice that if $I$ is finite, then $J$ is compact. If the system $S=\left\{\phi_{i}: X \rightarrow X: i \in I\right\}$, is pointwise finite (meaning that each element of $X$ belongs to at most finitely many elements of $\left.\phi_{i}(X)\right)$, then the family $\left\{\phi_{\omega}(X): \omega \in I^{n}\right\}$ is pointwise finite for every $n \geq 1$ and therefore

$$
J=\bigcap_{n=1}^{\infty} \bigcup_{\omega \in I^{n}} \phi_{\omega}(X)
$$

Thus $J$ is a $F_{\sigma \delta}$ subset of $X$. In Section 5 we will discuss examples of infinite i.f.s. whose associated limit sets are not $G_{\delta}$ subsets of $X$, equivalently which do not admit a complete metric. If the system $S$ is not assumed to be pointwise finite, then it seems that $J$ may even have much more complicated descriptive set theoretic structure. Let now $X(\infty)$ be the set of limit points of all sequences $x_{i} \in \phi_{i}(X), i \in I^{\prime}$, where $I^{\prime}$ ranges over all infinite subsets of $I$. As we shall see the geometric behavior of the system at this "asymptotic boundary" directly affects the geometric properties of the limit set $J$.

We shall prove the following.

Lemma 2.1. If $\lim _{i \in I} \operatorname{diam}\left(\phi_{i}(X)\right)=0$, then $\bar{J}=J \cup \bigcup_{\omega \in I^{*}} \phi_{\omega}(X(\infty))$.

Proof. First note that in view of (2.4) and the assumption, $X(\infty) \subset \bar{J}$. Therefore for every $i \in I$ we have $\phi_{i}(X(\infty)) \subset \phi_{i}(\bar{J}) \subset \overline{\phi_{i}(J)} \subset \bar{J}$. So, one inclusion is proved. In order to prove the other one consider $x \in \bar{J}$. Then there exists a sequence $\omega^{n}$ of points in $I^{\infty}$ such that $x=\lim _{n \rightarrow \infty} \pi\left(\omega^{n}\right)$. If the set of first coordinates of points $\omega^{n}$ is infinite, $x \in X(\infty)$ and we are done. Otherwise, there exists $u_{1} \in I$ such that the set $N_{1}=\left\{n \geq 1: \omega_{1}^{n}=u_{1}\right\}$ is infinite. If now the set of second coordinates of points $\omega^{n}$, $n \in N_{1}$, is infinite, $x \in \phi_{u_{1}}(X(\infty))$ and we are done again. Otherwise there exists $u_{2} \in I$ such that the set $N_{2}=\left\{n \in N_{1}: \omega_{2}^{n}=u_{2}\right\}$ is infinite. So, if we can stop this procedure after finitely many, say $n$, steps, we are done, since then $x \in \phi_{u_{1} u_{2} \ldots u_{n}}(X(\infty))$. Otherwise, using (2.2) we will produce a sequence $u \in I^{\infty} \operatorname{such}$ that $\operatorname{dist}\left(x, \phi_{\left.u\right|_{n}}(X)\right)$ tends to zero which implies that $x=\pi(u) \in J$.

An iterated function system $S=\left\{\phi_{i}: X \rightarrow X: i \in I\right\}$, is said to satisfy the Open Set Condition (abbreviated (OSC)) if there exists a nonempty open set $U \subset X$ (in the topology of X) such that $\phi_{i}(U) \subset U$ for every $i \in I$ and $\phi_{i}(U) \cap \phi_{j}(U)=\emptyset$ for every pair $i, j \in I, i \neq j$.

An iterated function system $S$ satisfying OSC, is said to be conformal (c.i.f.s.) if the following conditions are satisfied.

(2.6) $X$ is a compact connected subset of a euclidean space $\mathbb{R}^{d}$ and $U=\operatorname{Int}_{\mathbb{R}^{d}}(X)$.

(2.7) There exist $\alpha, l>0$ such that for every $x \in \partial X \subset \mathbb{R}^{d}$ there exists an open cone $\operatorname{Con}\left(x, u_{x}, \alpha, l\right) \subset \operatorname{Int}(X)$ with vertex $x$, direction vector $u_{x}$, central angle of Lebesgue measure $\alpha$, and altitude $l$.

(2.8) There exists an open connected set $X \subset V \subset \mathbb{R}^{d}$ such that all maps $\phi_{i}, i \in I$, extend to $C^{1+\varepsilon}$ diffeomorphisms on $V$ and are conformal on $V$. 
(2.9) Bounded Distortion Property (BDP). There exists $K \geq 1$ such that $\left|\phi_{\omega}^{\prime}(y)\right| \leq$ $K\left|\phi_{\omega}^{\prime}(x)\right|$ for every $\omega \in I^{*}$ and every pair of points $x, y \in V$, where $\left|\phi_{\omega}^{\prime}(x)\right|$ means the norm of the derivative.

Let us comment on conditions (2.7) and (2.9). First, although condition (2.7) may seem to be fairly sophisticated, in fact it is natural and it is satisfied for example if $\partial X$, the boundary of $X$, is smooth enough or it is convex. It should be also noticed that in fact the following weaker condition (see [12], p. 72)

$$
\inf _{x \in \partial X} \inf _{0<r<1} \lambda_{d}(B(x, r) \cap \operatorname{Int}(X)) / \lambda_{d}(B(x, r)>0
$$

would be sufficiently strong for our aims. We will suppress the direction vector $u_{x}$ in our notation for cones.

Now, to have a better understanding of what (BDP) actually says let us derive some of its geometric consequences. Indeed, it follows from (BDP) and the mean value inequality that

$$
\operatorname{diam}\left(\phi_{\omega}(B)\right) \leq\left\|\phi_{\omega}^{\prime}\right\| \operatorname{diam}(B) \text { and } \phi_{\omega}(B(x, r)) \subset B\left(\phi_{\omega}(x),\left\|\phi_{\omega}^{\prime}\right\| r\right)
$$

for all $\omega \in I^{*}$, all convex subsets $B$ of $V$, and all balls centered at points of $X$ with sufficiently small radii $(\leq \operatorname{dist}(X, \partial V))$. The norm is the supremum norm taken over $V$. However, by changing some constants we could take the norm over $X$. In order to get a similar estimate for the whole set $X$ it therefore suffices to notice that $X$ as a compact and connected set can be covered by a finite chain of balls $\left\{B\left(x_{j}, r_{j}\right): 1 \leq j \leq q\right\}$ (chain in the sense that $B\left(x_{j}, r_{j}\right) \cap B\left(x_{j+1}, r_{j+1}\right) \neq \emptyset$ for all $\left.j=1,2, \ldots, q-1\right)$ contained in $V$. In fact, decreasing $V$ to be the union of this chain (this operation preserves all the constraints imposed so far on $V$ ) we can write

$$
\operatorname{diam}\left(\phi_{\omega}(V)\right) \leq D\left\|\phi_{\omega}^{\prime}\right\|
$$

where $D \geq 1$ is any number $\geq q \operatorname{diam}(V)$. In a moment, for other purposes, we may need to take $D$ bigger than $q \operatorname{diam}(V)$.

Take now $x \in X$ and $0<r \leq \operatorname{dist}(X, \partial V)$. Then $B(x, r) \subset V$. Take also any $\omega \in I^{*}$ and let $R \geq 0$ be the maximal radius such that $B\left(\phi_{\omega}(x), R\right) \subset \phi_{\omega}(B(x, r))$. Then $\partial\left(B\left(\phi_{\omega}(x), R\right)\right) \cap \partial\left(\phi_{\omega}(B(x, r))\right) \neq \emptyset$, and in view of (BDP) we have $\phi_{\omega}^{-1}\left(B\left(\phi_{\omega}(x), R\right)\right) \subset$ $B\left(x,\left\|\phi_{\omega}^{-1}\right\| R\right) \subset B\left(x, K\left\|\phi_{\omega}^{\prime}\right\|^{-1} R\right)$. Therefore $K\left\|\phi_{\omega}^{\prime}\right\|^{-1} R \geq r$. Hence we have proved that

$$
\phi_{\omega}(B(x, r)) \supset B\left(\phi_{\omega}(x), K^{-1}\left\|\phi_{\omega}^{\prime}\right\| r\right)
$$

for every $x \in X, 0<r \leq \operatorname{dist}(X, \partial V)$, and $\omega \in I^{*}$. Taking now $\eta=\operatorname{dist}(X, \partial V)$ and assuming $D \geq K \eta^{-1}$, we can write

$$
\operatorname{diam}\left(\phi_{\omega}(X)\right) \geq D^{-1}\left\|\phi_{\omega}^{\prime}\right\| \text { and } \phi_{\omega}(X) \supset B\left(\phi_{\omega}(x), D^{-1}\left\|\phi_{\omega}^{\prime}\right\|\right)
$$


for all $\omega \in I^{*}$ and all $x \in X$. Note also that the above formula remains true if $X$ is replaced by the limit set $J$, perhaps with a larger value of $D$. In the sequel, when we use (BDP) we mean the original formula (2.9) and otherwise we will always indicate which one of its geometrical consequences we are using by the label. Combining (BDP) and (2.7) we conclude that if $D \geq 1$ is chosen large enough, then there exists $0<\beta \leq \alpha$ such that for all $x \in X$ and for all $\omega \in I^{*}$

$$
\phi_{\omega}(\operatorname{Int}(X)) \supset \operatorname{Con}\left(\phi_{\omega}(x), \beta, D^{-1}\left\|\phi_{\omega}^{\prime}\right\|\right) \supset \operatorname{Con}\left(\phi_{\omega}(x), \beta, D^{-2} \operatorname{diam}(X)\right)
$$

where $\operatorname{Con}\left(\phi_{\omega}(x), \beta, D^{-1}\left\|\phi_{\omega}^{\prime}\right\|\right)$ and $\operatorname{Con}\left(\phi_{\omega}(x), \beta, D^{-2} \operatorname{diam}(X)\right)$ denote some cones with vertices at $\phi_{\omega}(x)$, angles $\beta$, and altitudes $D^{-1}\left\|\phi_{\omega}^{\prime}\right\|$ and $D^{-2} \operatorname{diam}(X)$ respectively.

We feel also that a discussion about how to check condition (2.9) in practice would be in order, and this is done in the next lemma and two remarks following it.

Lemma 2.2. Each of the following three conditions is sufficient for (BDP) to hold.

(a) There are two constants $L \geq 1$ and $\alpha>0$ such that

$$
|| \phi_{i}^{\prime}(y)|-| \phi_{i}^{\prime}(x)|| \leq L||\left(\phi_{i}^{\prime}\right)^{-1} \|^{-1}|y-x|^{\alpha},
$$

for every $i \in I$ and every pair of points $x, y \in V$.

(b) For every $t>0$ let

$$
M(t)=\sup _{i \in I} \sup \left\{\|\left(\phi_{i}^{\prime}\right)^{-1}|||| \phi_{i}^{\prime}(y)|-| \phi_{i}^{\prime}(x)||:|y-x| \leq t\right\}<\infty,
$$

moreover the series $\Sigma(t):=\sum_{n>0} M\left(s^{n} t\right)$ converges and $\lim _{t \rightarrow 0} \Sigma(t)=0$.

(c) The family $\left\{\log \left|\phi_{\omega}^{\prime}\right|: \omega \in I^{*}\right\}$ of functions defined on $V$ is equicontinuous.

In fact we have the following chain of implications: $(a) \Rightarrow(b) \Rightarrow(c) \Rightarrow(B D P)$.

Proof. $((a) \Rightarrow(b))$. Indeed, for every $t>0$ we have $M(t) \leq L t^{\alpha}$ and therefore

$$
\sum_{n \geq 0} M\left(s^{n} t\right) \leq L t^{\alpha} \sum_{n \geq 0} s^{\alpha n}=L t^{\alpha}\left(1-s^{\alpha}\right)^{-1}
$$

where $s$ is the bound on the contraction ratios given in (2.1), which finishes the proof of this implication.

$((b) \Rightarrow(c))$. Let $\omega \in I^{*}$ and let $n=|\omega|$. For every $z \in V$ and every $k=1,2, \ldots, n$ define $z_{k}=\phi_{\omega_{n-k+1}} \circ \phi_{\omega_{n-k+2}} \circ \ldots \circ \phi_{\omega_{n}}(z)$; put also $z_{0}=z$. Fix $\varepsilon>0$ and take $\delta>0$ so small that $\Sigma(\delta)<\varepsilon$. Take any two points $x, y \in V$ with $|y-x|<\delta$. Then

$$
\begin{aligned}
\left|\log \left(\left|\phi_{\omega}^{\prime}(y)\right|\right)-\log \left(\left|\phi_{\omega}^{\prime}(x)\right|\right)\right| & =\left|\sum_{j=1}^{n} \log \left(1+\frac{\left|\phi_{\omega_{j}}^{\prime}\left(y_{n-j}\right)\right|-\left|\phi_{\omega_{j}}^{\prime}\left(x_{n-j}\right)\right|}{\left|\phi_{\omega_{j}}^{\prime}\left(x_{n-j}\right)\right|}\right)\right| \\
& \leq \sum_{j=1}^{n}||\left(\phi_{\omega_{j}}^{\prime}\right)^{-1}|||| \phi_{\omega_{j}}^{\prime}\left(y_{n-j}\right)|-| \phi_{\omega_{j}}^{\prime}\left(x_{n-j}\right)|| \\
& \leq \sum_{j=1}^{n} M\left(s^{(n-j)} \delta\right) \leq \sum_{k=0}^{n-1} M\left(s^{k} \delta\right)<\varepsilon .
\end{aligned}
$$


$((c) \Rightarrow(\mathrm{BDP}))$. In view of the equicontinuity, there is $\delta>0$ such that if $|y-x| \leq \delta$, then $\left|\log \left(\left|\phi_{\omega}^{\prime}(y)\right|\right)-\log \left(\left|\phi_{\omega}^{\prime}(x)\right|\right)\right| \leq 1$ for all $\omega \in I^{*}$. Since $X$ is compact and connected, there is a finite chain, say of cardinality $n$, of balls with radii $\delta / 2$ whose union, $W$, contains $X$. Therefore if $x$ and $y$ are two arbitrary points in $W$, then there is a sequence $\left\{z_{i}: i=\right.$ $1, \ldots, k\}, z_{1}=y, z_{k}=x, k \leq n+1$ of points in $W$ such that $\left|z_{i+1}-z_{i}\right|<\delta$ for all $i=$ $1, \ldots, k-1$. Hence $\left|\log \left(\left|\phi_{\omega}^{\prime}(y)\right|\right)-\log \left(\left|\phi_{\omega}^{\prime}(x)\right|\right)\right| \leq \sum_{i=1}^{k-1}\left|\log \left(\left|\phi_{\omega}^{\prime}\left(z_{i}\right)\right|\right)-\log \left(\left|\phi_{\omega}^{\prime}\left(z_{i+1}\right)\right|\right)\right| \leq$ $k-1$. Thus $\left|\phi_{\omega}^{\prime}(y)\right| /\left|\phi_{\omega}^{\prime}(x)\right| \leq e^{n}$, which with $V$ shrunk to $W$ finishes the proof of this implication and the whole lemma.

Remark 2.3. The assumption of Lemma 2.2 is obviously satisfied if the alphabet $I$ is finite and all the maps $\phi_{i}, i \in I$, belong to the class $C^{1+\varepsilon}$. So (BDP) holds in this case.

Remark 2.4. Because of the Koebe distortion theorem (see [23]), (BDP) is automatically satisfied if $X$ is a subset of the plane.

From now on throughout the paper we will be actually interested only in conformal systems. We begin to explore them by proving the following.

Lemma 2.5. If $S$ is an infinite c.i.f.s., then

$$
\sum_{i \in I}\left\|\phi_{i}^{\prime}\right\|^{d} \leq K^{d} \text { and } \lim _{i \in I} \operatorname{diam}\left(\phi_{i}(X)\right)=0
$$

Proof. For each $i \in I$ denote by $J_{\phi_{i}}$ the Jacobian of the contraction $\phi_{i}$. By conformality $\left|J_{\phi_{i}}\right|=\left|\phi_{i}^{\prime}\right|^{d}$. Thus by (OSC) and (BDP) we get

$$
\lambda_{d}(\operatorname{Int}(X)) \geq \sum_{i \in I} \lambda_{d}\left(\operatorname{Int}\left(\phi_{i}(X)\right)\right)=\sum_{i \in I} \int_{\operatorname{Int}(X)}\left|J_{\phi_{i}}\right| d \lambda_{d} \geq \sum_{i \in I} K^{-d} \| \phi_{i}^{\prime} \mid{ }^{d} \lambda_{d}(\operatorname{Int}(X)) .
$$

Hence $\sum_{i \in I}\left\|\phi_{i}^{\prime}\right\|^{d} \leq K^{d}$ and therefore the lemma follows from (2.10).

There are two cardinality bounds arising from our geometric condition (2.7) which play a crucial role in our theory. To formulate them, for every $x \in X$ and every integer $n \geq 1$, let $\pi_{n}^{-1}(x)$ be the maximal collection of all mutually incomparable (meaning neither word is an extension of the other) words $\omega$ in $\bigcup_{j<n} I^{j}$ with $x \in \phi_{\omega}(X)$ such that if $\omega, \tau \in \pi_{n}^{-1}(x)$ and $\tau$ is an extension of $\omega$, then $\tau=\omega$. From (2.10) we immediately get the following.

Lemma 2.6. If $S$ is a c.i.f.s., then for every $x \in X$ and every integer $n \geq 1$, we have $\# \pi_{n}^{-1}(x) \leq \lambda_{d-1}\left(S^{d-1}\right) / \beta$. In particular, $S$ is uniformly pointwise finite. More precisely $\sup _{x \in X} \#\left\{i \in I: x \in \phi_{i}(X)\right\} \leq \lambda_{d-1}\left(S^{d-1}\right) / \beta<\infty$.

Formula (2.10) also implies the following.

Lemma 2.7. If $S$ is a c.i.f.s., then for every $x \in X$ and every $r>0$, the cardinality of any collection of mutually incomparable words $\omega \in I^{*}$ satisfying $B(x, r) \cap \phi_{\omega}(X) \neq \emptyset$ and $\operatorname{diam}\left(\phi_{\omega}(X)\right) \geq r$ is bounded from above by the number $V_{d} D^{2 d} \beta^{-1}\left(1+D^{-2}\right)$. 
Proof. Let $F$ be such a family. It follows from (2.10) that

$$
\phi_{\omega}(X) \supset \operatorname{Con}\left(\phi_{\omega}\left(x_{\omega}\right), \beta, D^{-2} r\right) \subset B\left(x,\left(1+D^{-2}\right) r\right)
$$

for all $\omega \in F$, where $\phi_{\omega}\left(x_{\omega}\right)$ is on the boundary of $\phi_{\omega}(X) \cap B(x, r)$. Hence the cones $\operatorname{Con}\left(\phi_{\omega}\left(x_{\omega}\right), \beta, D^{-2} r\right), \omega \in F$, are mutually disjoint and therefore $V_{d}\left(1+D^{-2}\right) r^{d}=$ $\lambda_{d}\left(B\left(x,\left(1+D^{-2}\right) r\right)\right) \geq \sum_{\omega \in F} \lambda_{d}\left(\operatorname{Con}\left(\phi_{\omega}\left(x_{\omega}\right), \beta, D^{-2} r\right)\right)=\# F \beta\left(D^{-2} r\right)^{d}$. Now, the required estimate follows.

Let us remark here that that infinite c.i.f.s are not necessarily uniformly locally finite. Several examples (e.g., Example 5.1) given in Section 5 are not even locally finite. Some of them however are and the complex continued fraction example described in Section 6 is uniformly locally finite.

In all following sections we will extensively use the concepts of conformal and semiconformal measures. The definition of semiconformal measures is somewhat technical and is postponed to the next section. The definition of conformal measures is simpler, more important, and will be provided here. So, given $t \geq 0$ a Borel probability measure $m$ is said to be $t$-conformal provided $m(J)=1$ and for every Borel set $A \subset X$ and every $i \in I$

$$
m\left(\phi_{i}(A)\right)=\int_{A}\left|\phi_{i}^{\prime}\right|^{t} d m
$$

and

$$
m\left(\phi_{i}(X) \cap \phi_{j}(X)\right)=0,
$$

for every pair $i, j \in I, i \neq j$. Then an easy computation shows that for every Borel set $A \subset X$ and every $\omega \in I^{*}$

$$
m\left(\phi_{\omega}(A)\right)=\int_{A}\left|\phi_{\omega}^{\prime}\right|^{t} d m
$$

and

$$
m\left(\phi_{\omega}(X) \cap \phi_{\tau}(X)\right)=0,
$$

for every pair $\omega, \tau \in I^{*}$ of incomparable (neither word is an extension of the other) words. As an immediate consequence of this definition and (BDP) we get the following: if $m$ is $\delta$-conformal, then

$$
1 \leq \sum_{\omega \in I^{n}}\left|\phi_{\omega}^{\prime}\right|^{\delta} \leq K^{\delta} .
$$

Let us conclude this section with some general facts from geometric measure theory. Given a subset $A$ of a compact metric space $(X, d)$, a countable family $\left\{B\left(x_{i}, r_{i}\right)\right\}_{i=1}^{\infty}$ of open balls centered at points of $A$ is said to be a packing of $A$ if and only if for any pair $i \neq j$

$$
d\left(x_{i}, x_{j}\right) \geq r_{i}+r_{j} .
$$


Given a nondecreasing function $g:(0, \varepsilon) \rightarrow(0, \infty)$ for some $\varepsilon>0$, the $g$-dimensional outer Hausdorff measure $\mathrm{H}_{g}(A)$ of the set $A$ is defined as

$$
\mathrm{H}_{g}(A)=\sup _{\varepsilon>0} \inf \left\{\sum_{i=1}^{\infty} g\left(\operatorname{diam}\left(A_{i}\right)\right)\right\}
$$

where the infimum is taken over all countable covers $\left\{A_{i}: i \geq 1\right\}$ of $A$ by arbitrary sets whose diameters do not exceed $\varepsilon$. If $g$ is of the form $x^{t}$ instead of writing $\mathrm{H}_{x^{t}}$ we write $\mathrm{H}_{t}$ and speak about $t$-dimensional outer Hausdorff measure. In this case one will get comparable numbers (in the sense that ratios are bounded away from zero and infinity) if instead of covering $A$ by arbitrary sets one considers only open balls centered at points of A.

The $g$-dimensional outer packing measure $\Pi_{g}(A)$ of the set $A$ is defined as

$$
\Pi_{g}(A)=\inf _{\cup A_{i}=A}\left\{\sum_{i} \Pi_{g}^{*}\left(A_{i}\right)\right\}
$$

$\left(A_{i}\right.$ are arbitrary subsets of $\left.A\right)$, where $\Pi_{g}^{*}$, the $g$-packing premeasure is given by:

$$
\Pi_{g}^{*}(A)=\inf _{\varepsilon>0} \sup \left\{\sum_{i=1}^{\infty} g\left(2 r_{i}\right)\right\}
$$

Here the supremum is taken over all packings $\left\{B\left(x_{i}, r_{i}\right)\right\}_{i=1}^{\infty}$ of the set $A$ by open balls centered at points of $A$ with radii which do not exceed $\varepsilon$. Similarly as in the case of Hausdorff measures if $g$ is of the form $x^{t}$ instead of writting $\Pi_{x^{t}}$ we write $\Pi_{t}$ and speak about $t$-dimensional outer packing measure. These two outer measures $\mathrm{H}_{g}$ and $\Pi_{g}$ define countable additive measures on Borel $\sigma$-algebra of $X$. For additional properties of packing measures and a comprehensive discussion of this and related notions the reader is referred to the paper [35].

The definitions of the Hausdorff dimension $\operatorname{HD}(A)$ of $A$ and packing dimension $\mathrm{PD}(A)$ are the following

$$
\mathrm{HD}(A)=\inf \left\{t: \mathrm{H}_{t}(A)=0\right\}=\sup \left\{t: \mathrm{H}_{t}(A)=\infty\right\}
$$

and

$$
\operatorname{PD}(A)=\inf \left\{t: \Pi_{t}(A)=0\right\}=\sup \left\{t: \Pi_{t}(A)=\infty\right\} .
$$

Moreover we shall deal with lower box dimension $\underline{\mathrm{BD}}(A)$ and upper box dimension $\overline{\mathrm{BD}}(A)$ which are respectively defined as follows.

$$
\underline{\mathrm{BD}}(A)=\liminf _{r \rightarrow 0} \frac{\log N(A, r)}{-\log r} \text { and } \overline{\mathrm{BD}}(A)=\limsup _{r \rightarrow 0} \frac{\log N(A, r)}{-\log r},
$$

where $N(A, r)$ is the minimal number of balls with radii $\leq r$ needed to cover $A$. If the lower and upper box dimensions coincide, then their common value $\mathrm{BD}(A)=\underline{\mathrm{BD}}(A)=\overline{\mathrm{BD}}(A)$ is 
called the box dimension of $A$. In the literature all box dimensions are also called, perhaps more properly, box counting dimensions; we will keep here the shorter name. We recall (see [9] for example) that $\mathrm{HD}(A) \leq \mathrm{PD}(A) \leq \overline{\mathrm{BD}}(A)$ and $\mathrm{HD}(A) \leq \underline{\mathrm{BD}}(A)=\underline{\mathrm{BD}}(\bar{A}) \leq$ $\overline{\mathrm{BD}}(A)=\overline{\mathrm{BD}}(\bar{A})$.

Let $\nu$ be a Borel probability measure on $X$ and let $t \geq 0$ be a real number. Define the function $\rho=\rho_{t}(\nu): X \times(0, \infty) \rightarrow(0, \infty)$ by

$$
\rho(x, r)=\frac{\nu(B(x, r))}{r^{t}} .
$$

The following two theorems are for our aims the key facts from geometric measure theory. The proof of the first one follows from the results obtained in [25] and the proof of the second one is contained in [35]. Proofs may be found in [MAT].

Theorem 2.8. Assume that $X$ is a compact subspace of a d-dimensional euclidean space. Then for every $t \geq 0$ there exist constants $h_{1}(t)$ and $h_{2}(t)$ with the following properties: If $A$ is a Borel subset of $X$ and $C>0$ is a constant such that

(1) for all (but countably many) $x \in A$

$$
\limsup _{r \rightarrow 0} \rho(x, r) \geq C^{-1}
$$

then for every Borel subset $E \subset A$ we have $H_{t}(E) \leq h_{1}(t) C \nu(E)$ and, in particular, $H_{t}(A)<\infty$.

or

(2) for all $x \in A$

$$
\limsup _{r \rightarrow 0} \rho(x, r) \leq C^{-1}
$$

then for every Borel subset $E \subset A$ we have $H_{t}(E) \geq C h_{2}(t) \nu(E)$.

Theorem 2.9. Assume that $X$ is a compact subspace of an d-dimensional euclidean space. Then there exist constants $p_{1}(t)$ and $p_{2}(t)$ with the following properties: If $A$ is a Borel subset of $X$ and $C>0$ is a constant such that

(1) for all $x \in A$

$$
\liminf _{r \rightarrow 0} \rho(x, r) \leq C^{-1},
$$

then for every Borel subset $E \subset A$ we have $\Pi_{t}(E) \geq C p_{1}(t) \nu(E)$, or

(2) for all $x \in A$

$$
\liminf _{r \rightarrow 0} \rho(x, r) \geq C^{-1},
$$

then for every Borel subset $E \subset A$ we have $\Pi_{t}(E) \leq p_{2}(t) C \nu(E)$ and, consequently, $\Pi_{t}(A)<\infty$.

(1') If $\nu$ is non-atomic, then (1) holds under the weaker assumption that the hypothesis of part (1) is satisfied on the complement of a countable set. 


\section{Pressure, measures, and dimensions}

In this section we introduce topological pressure, Perron-Frobenius operator, and conformal and semiconformal measures. We define and study here the class of regular and hereditarily regular systems, and using a symbolic representation of the limit set we prove the existence and uniqueness of the conformal measure. We also derive the existence of an invariant probability equivalent to the conformal measure. Proving its ergodicity we simultaneously show its uniqueness. At the end of the section we give a proof that if the system has finite entropy, then the Hausdorff dimension of the conformal measure is equal to the Hausdorff dimension of the limit set.

Let us begin this section with the following mutual equality of all box and packing dimensions of the limit set $J$ and its closure $\bar{J}$ for iterated function systems.

Theorem 3.1. If $S$ is an i.f.s. and all maps $\phi_{i}$ are bi-Lipschitz, then $P D(J)=$ $\overline{B D}(J)=P D(\bar{J})=\overline{B D}(\bar{J})$.

Proof. The inequalities $\mathrm{PD}(J) \leq \mathrm{PD}(\bar{J}) \leq \overline{\mathrm{BD}}(\bar{J})$ and $\mathrm{PD}(J) \leq \overline{\mathrm{BD}}(J) \leq \overline{\mathrm{BD}}(\bar{J})$ are obvious. Thus to complete the proof it suffices to show that $\operatorname{PD}(J) \geq \overline{\operatorname{BD}}(J)$. Indeed, fix $t<\overline{\mathrm{BD}}(J)$ and consider an arbitrary countable cover $\left\{Y_{n}: n \geq 1\right\}$ of $J$. Since the metric space $I^{\infty}$ is complete, there exists $q \geq 1$ such that $\pi^{-1}\left(\overline{Y_{q}}\right)$ has nonempty interior in $I^{\infty}$. Therefore there exists an $\omega \in I^{*}$ such that $\{\omega\} \times I^{\infty} \subset \pi^{-1}\left(\overline{Y_{q}}\right)$, whence $\phi_{\omega}(J)=\pi\left(\{\omega\} \times I^{\infty}\right) \subset \overline{Y_{q}}$. Since $t<\overline{\mathrm{BD}}(J)$, we have $\Pi_{t}^{*}(J)=\infty$. Since $\phi_{\omega}$ is biLipschitz, we therefore find $\Pi_{t}^{*}\left(Y_{q}\right)=\Pi_{t}^{*}\left(\phi_{\omega}\left(Y_{q}\right)\right)=\infty$. Thus $\sum_{n \geq 1} \Pi_{t}^{*}\left(Y_{n}\right)=\infty$ and consequently $\Pi_{t}(J)=\infty$ which completes the proof.

In Example 5.2 we show that the inequality $\mathrm{HD}(J)<\mathrm{PD}(J)$ can occur even in conformal systems. Now let us pass to study conformal systems. Until Lemma 3.10, unless otherwise stated, we do not assume that (OSC) and (2.7) hold. For every $t \geq 0$ consider the series

$$
\psi(t)=\sum_{i \in I}\left\|\phi_{i}^{\prime}\right\|^{t}
$$

Let $\theta=\theta_{S}=\inf \{t: \psi(t)<\infty\} \geq 0$ and let $F(S)$ be the set of finiteness of $\psi$, so either $F(S)=(\theta, \infty)$ or $F(S)=[\theta, \infty)$. Some elementary properties of $\psi$ are collected in the following lemma.

Lemma 3.2. The function $\psi(t)$ is nonincreasing. It is strictly decreasing on $[\theta, \infty)$, and continuous and log convex on $F(S)$. Additionally, $\psi(d) \leq K^{d}$ which implies that $\theta \leq d$.

This lemma is actually obvious. That $\psi$ is $\log$ convex follows from an application of Hölder's inequality, and the last assertion is included in Lemma 2.5. Note that $\psi(\theta)$ may be infinite as well as finite - in Examples 5.3 and 5.4 we have $\psi(\theta)<\infty$, on the other hand in Examples 5.5 and $5.6 \psi(\theta)=\infty$ and this is also the case for complex continued fractions described in Section 6. This dichotomy plays an important role in the classification of c.i.f.s into regular and irregular systems as explained in the last six results 
of this section. Notice also that, as Example 5.3 shows, $\theta$ may be $d$. For every integer $n \geq 1$ define $\psi_{n}(t)=\sum_{\omega \in I^{n}}\left\|\phi_{\omega}^{\prime}\right\|^{t}$ (so $\psi=\psi_{1}$ ). By (BDP) for all integers $k, n \geq 1$ we have $K^{-t} \psi_{k}(t) \psi_{n}(t) \leq \psi_{k+n}(t) \leq \psi_{k}(t) \psi_{n}(t)$. It follows from this that $\psi_{n}(t)<\infty$ for all $n \geq 1$ if and only if $\psi_{n}(t)<\infty$ for some $n \geq 1$ if and only if $\psi(t)<\infty$. An application of Hölder's inequality shows that each function $\psi_{n}$ is log convex. These facts imply that the following limit

$$
\mathrm{P}(t)=\lim _{n \rightarrow \infty} \frac{1}{n} \log \psi_{n}(t)=\lim _{n \rightarrow \infty} \frac{1}{n} \log \sum_{\omega \in I^{n}}\left\|\phi_{\omega}^{\prime}\right\|^{t}
$$

always exists and is finite if and only if $\psi(t)<\infty$, in particular $\inf \{t: \mathrm{P}(t)<\infty\}=\theta_{S}$. Note also that in view of (BDP) for every $x \in V$

$$
\mathrm{P}(t)=\lim _{n \rightarrow \infty} \frac{1}{n} \log \sum_{\omega \in I^{n}}\left|\phi_{\omega}^{\prime}(x)\right|^{t} .
$$

We call $\mathrm{P}(t)$ the topological pressure of the system $S$. For an exposition of the theory of this notion in the context of dynamical systems acting on a compact metric space the reader is referred to the books [26] and [39] by D. Ruelle and P. Walters respectively and to the articles [37] and [38] for example. The following proposition collects some of basic properties of topological pressure.

Proposition 3.3. $\inf \{t: \psi(t)<\infty\}=\theta_{S}$. The topological pressure function $P(t)$ is nonincreasing on $[0, \infty)$, strictly decreasing on $[\theta, \infty)$, convex and continuous on $F(S)$. Additionally $P(0)=\infty$ if and only if $I$ is infinite.

Proof. The first statement of this proposition has been proved above. The facts that the pressure function $\mathrm{P}(t)$ is nonincreasing on $[0, \infty)$ and is strictly decreasing on $[\theta, \infty)$ are obvious. Since $\mathrm{P}$ is the limit of convex functions on $F(S), \mathrm{P}$ is convex on $F(S)$. This in turn implies that $\mathrm{P}(t)$ is continuous on $F(S)$.

Now we shall study semiconformal and conformal measures. First without assuming (OSC) and (2.7), we define semiconformal measures and prove some of their basic properties. Next, assuming (OSC) and (2.7), we prove the existence of conformal measures and establish some geometric properties of conformal systems. Our way of dealing with semiconformal measures is motivated by the approach given in [38] where a more complete collection of references can be found.

Suppose that $\delta \in F(S)$ and for every bounded function $f: X \rightarrow \mathbb{R}$ put

$$
\mathcal{L}_{\delta}(f)(x)=\sum_{i \in I}\left|\phi_{i}^{\prime}(x)\right|^{\delta} f\left(\phi_{i}(x)\right)
$$

Notice that $\mathcal{L}_{\delta}$ preserves the space of continuous functions $C(X)$ and that its norm is bounded by $\psi(\delta)$, so it is continuous. Denote by $\mathcal{L}_{\delta}^{*}: C(X)^{*} \rightarrow C(X)^{*}$ its dual operator. We shall prove the following.

Lemma 3.4. If $t \geq 0$ and $m$ is a t-conformal measure, then $t \in F(S)$ (even more, $P(t)=0$ ) and $\mathcal{L}_{t}^{*}(m)=m$. 
Proof. It follows immediately from (2.15) that $t \in F(S)$ and $\mathrm{P}(t)=0$. Moreover for each $f \in C(X)$ we have $\mathcal{L}_{t}^{*} m(f)=\int \mathcal{L}_{t}(f) d m=\int \sum_{i \in I}\left|\phi_{i}^{\prime}\right|^{t}\left(f \circ \phi_{i}\right) d m=$ $\sum_{i \in I} \int_{\phi_{i}(X)} f d m=\int \oint_{\phi_{i}(X)} f d m=\int f d m=m(f)$. The proof is finished.

This lemma gives us the first motivation to distinguish probability measures that are fixed points of the dual operator $\mathcal{L}_{\delta}^{*}$ and to name them $\delta$-semiconformal measures. Another reason is, as we have already mentioned, that $\delta$-semiconformal measures are proven to exist even if (OSC) is not satisfied and a conformal measure may fail to exist. Moreover, the semiconformal themselves carry interesting geometric and dynamic information. So, our next aim is to show the existence of semiconformal measures. Frequently, if it does not lead to a misunderstanding we will drop the subscript $\delta$ and simply write $\mathcal{L}$ and $\mathcal{L}^{*}$ for $\mathcal{L}_{\delta}$ and $\mathcal{L}_{\delta}^{*}$ respectively. We begin with the following.

Theorem 3.5. A $\delta$-semiconformal measure exists if and only if $P(\delta)=0$. Moreover, if $m$ is $\delta$-semiconformal, then $m(J)=1$.

Proof. Suppose first that a $\delta$-semiconformal measure exists. Call it $m$. Then for every $n \geq 1$ we have $1=\int 1 d m=\int \mathcal{L}^{n}(1) d m=\int \sum_{i \in I^{n}}\left|\phi_{\omega}^{\prime}\right|^{\delta} d m$ and using (BDP) we conclude that $1 \leq \sum_{i \in I^{n}}\left\|\phi_{\omega}^{\prime}\right\|^{\delta} \leq K^{\delta}$. Hence $\mathrm{P}(\delta)=0$ which finishes the first part of the proof.

Now suppose that $\mathrm{P}(\delta)=0$. Consider the continuous map $\nu \rightarrow \mathcal{L}^{*}(\nu) / \mathcal{L}^{*}(\nu)(1)$ defined on the space of Borel probability measures on $X$ treated as a subspace of $C(X)^{*}$. In view of Schauder-Tichonov theorem this map has a fixed point, say $m$. Writing $\lambda=$ $\mathcal{L}^{*}(m)(1)$ we thus have $\mathcal{L}^{*}(m)=\lambda m$. We shall now show that $\lambda=1$. Indeed, since $\mathcal{L}^{n}(f)(x)=\sum_{\omega \in I^{n}}\left|\phi_{\omega}^{\prime}(x)\right|^{\delta} f\left(\phi_{\omega}(x)\right)$ for all $n \geq 1$, we get $\left(\mathcal{L}^{*}\right)^{n}(m)=\lambda^{n} m$ which implies that $\int \sum_{\omega \in I^{n}}\left|\phi_{\omega}^{\prime}\right|^{\delta} d m=\lambda^{n} \int 1 d m=\lambda^{n}$. On the other hand since $\mathrm{P}(\delta)=0$, using (BDP) we conclude that for every $\varepsilon>0$, every $n \geq 1$ large enough and every $x \in X$ we have $e^{-\varepsilon n} \leq \sum_{\omega \in I^{n}}\left|\phi_{\omega}^{\prime}(x)\right|^{\delta} \leq e^{\varepsilon n}$ and consequently $e^{-\varepsilon n} \leq \lambda^{n} \leq e^{\varepsilon n}$. Thus $\lambda=1$, so $m$ is a fixed point of $\mathcal{L}^{*}$.

Now, suppose $m$ is $\delta$-semiconformal or equivalently,

$$
\int \sum_{\omega \in I^{n}}\left|\phi_{\omega}^{\prime}\right|^{\delta}\left(f \circ \phi_{\omega}\right) d m=\int f d m
$$

for every continuous function $f: X \rightarrow \mathbb{R}$. Since this equality extends to all bounded measurable functions $f$, we get

$$
m\left(\phi_{\omega}(A)\right)=\sum_{\tau \in I^{n}} \int\left|\phi_{\tau}^{\prime}\right|^{\delta}\left(1_{\phi_{\omega}(A)} \circ \phi_{\tau}\right) d m \geq \int_{A}\left|\phi_{\omega}^{\prime}\right|^{\delta} d m
$$

for all $\omega \in I^{n}$ and all Borel subsets $A$ of $X$. Now for each $n \geq 1$ set $X_{n}=\bigcup_{\omega \in I^{n}} \phi_{\omega}(X)$. Then $1_{X_{n}} \circ \phi_{\omega}=1$ for all $\omega \in I^{n}$. Thus applying (3.1) to the function $f=1_{X_{n}}$ and later to the function $f=1$ we obtain $m\left(X_{n}\right)=\int \sum_{\omega \in I^{n}}\left|\phi_{\omega}^{\prime}\right|^{\delta}\left(1_{X_{n}} \circ \phi_{\omega}\right) d m=\int \sum_{\omega \in I^{n}}\left|\phi_{\omega}^{\prime}\right|^{\delta} d m=$ $\int 1 d m=1$. Hence, $m(J)=m\left(\bigcap_{n \geq 1} X_{n}\right)=1$. The proof is finished. 
Observe that slightly more generally, if $t \in F(S)$, then the same proof leads to the existence of a probability measure $m_{t}$ on $J$ such that $\mathcal{L}^{*}\left(m_{t}\right)=e^{\mathrm{P}(t)} m_{t}$.

For the remainder of the section we assume that $\mathrm{P}(\delta)=0$ and $m$ is a $\delta$-semiconformal measure. Frequently to stress its importance we will repeat this assumption in the formulations of our results. Let us also make some additional notation. If $\omega \in I^{*}$, set $[\omega]=\left\{\tau \in I^{\infty}:\left.\tau\right|_{|\omega|}=\omega\right\}$. We shall prove the following.

Lemma 3.6. There exists a unique Borel probability measure $\mu$ on $I^{\infty}$ such that $\mu([\omega])=\int\left|\phi_{\omega}^{\prime}\right|^{\delta} d m$ for all $\omega \in I^{*}$.

Proof. In view of (3.1), $\sum_{\omega \in I^{n}} \int\left|\phi_{\omega}^{\prime}\right|^{\delta} d m=1$ for all $n \geq 1$ and therefore one can define a Borel probability measure $\mu_{n}$ on $C_{n}$, the algebra generated by the cylinder sets of the form $[\omega]$, where $\omega \in I^{n}$, by putting $\mu_{n}([\omega])=\int\left|\phi_{\omega}^{\prime}\right|^{\delta} d m$. Hence applying (3.1) again we get for all $\omega \in I^{n}$

$$
\begin{aligned}
\mu_{n+1}([\omega]) & =\sum_{i \in I} \mu_{n+1}([\omega i])=\sum_{i \in I} \int\left|\phi_{\omega i}^{\prime}\right|^{\delta} d m=\int \sum_{i \in I}\left(\left|\phi_{\omega}^{\prime}\right| \circ \phi_{i}\right)^{\delta}\left|\phi_{i}^{\prime}\right|^{\delta} d m \\
& =\int\left|\phi_{\omega}^{\prime}\right|^{\delta} d m=\mu_{n}([\omega])
\end{aligned}
$$

and therefore, in view of Kolmogorov's extension theorem there exists a unique probability measure $\mu$ on $I^{\infty}$ such that $\mu([\omega])=\mu_{|\omega|}([\omega])$ for all $\omega \in I^{*}$.

As an immediate corollary of this lemma and (BDP) we see that if $R \subset I^{*}$ is a collection of incomparable words such that $\bigcup_{\omega \in R} \phi_{\omega}(X) \supset J$, then

$$
1 \leq \sum_{\omega \in R}\left\|\phi_{\omega}^{\prime}\right\|^{\delta} \leq K^{\delta}
$$

Now we shall prove the following.

Lemma 3.7. The measures $m$ and $\mu \circ \pi^{-1}$ are equal.

Proof. Let $A \subset J$ be an arbitrary closed subset of $J$ and for every $n \geq 1$ let $A_{n}=\left\{\omega \in I^{n}: \phi_{\omega}(X) \cap A \neq \emptyset\right\}$. In view of (3.1) applied to the characteristic function $1_{A}$ we have for all $n \geq 1$

$$
\begin{aligned}
m(A) & =\sum_{\omega \in I^{n}} \int\left|\phi_{\omega}^{\prime}(x)\right|^{\delta}\left(1_{A} \circ \phi_{\omega}\right) d m=\sum_{\omega \in A_{n}} \int\left|\phi_{\omega}^{\prime}(x)\right|^{\delta}\left(1_{A} \circ \phi_{\omega}\right) d m \\
& \leq \sum_{\omega \in A_{n}} \int\left|\phi_{\omega}^{\prime}(x)\right|^{\delta} d m=\sum_{\omega \in A_{n}} \mu([\omega])=\mu\left(\bigcup_{\omega \in A_{n}}[\omega]\right)
\end{aligned}
$$

Since the family of sets $\left\{\bigcup_{\omega \in A_{n}}[\omega]: n \geq 1\right\}$ is decreasing and, by Lemma 3.6, the cardinality of the sets $A_{n}$ are uniformly bounded and $\bigcap_{n \geq 1} \bigcup_{\omega \in A_{n}}[\omega]=\pi^{-1}(A)$, we therefore get $m(A) \leq \lim _{n \rightarrow \infty} \mu\left(\bigcup_{\omega \in A_{n}}[\omega]\right)=\mu\left(\pi^{-1}(A)\right)$. Since both measures $m$ and $\mu \circ \pi^{-1}$ are 
regular (as $J$ is a metric separable space), this inequality extends to the family of all Borel subsets of $J$. Since both measures are probabilistic we get $m=\mu \circ \pi^{-1}$. The proof is finished.

Let us recall that in the beginning of Section 2 by $\sigma: I^{\infty} \rightarrow I^{\infty}$ we have denoted the left shift map (cutting out the first coordinate) on $I^{\infty}$. Now we shall prove it has a unique invariant (ergodic) probability measure equivalent with $\mu$. For the sake of completeness we provide the reader with a complete proof although after noting that for all $\omega, \tau, \rho \in I^{*}$

$$
\frac{\mu([\omega \tau]) / \mu([\tau])}{\mu([\omega \rho]) / \mu([\rho])} \leq K^{\delta}
$$

we could apply Renyi's theorem (see [24], comp. [30]).

Theorem 3.8. There exists a unique ergodic $\sigma$-invariant probability measure $\mu^{*}$ absolutely continuous with respect to $\mu$. Moreover $\mu^{*}$ is equivalent with $\mu$ and $K^{-\delta} \leq$ $d \mu^{*} / d \mu \leq K^{\delta}$.

Proof. Let $L$ be a Banach limit defined on the Banach space of all bounded sequences of real numbers. Straightforward computations and an application of Kolmogorov's extension theorem show that the function $\mu^{*}([\omega])=L\left(\left(\mu\left(\sigma^{-n}([\omega])\right)\right)_{n \geq 0}\right)$ defined on $I^{*}$, extends to a $\sigma$-invariant probability measure on $I^{\infty}$. Keep for it the same symbol $\mu^{*}$. Notice that, using (3.3), for each $\omega \in I^{*}$ and each $n \geq 0$ we have

$$
\begin{aligned}
\mu\left(\sigma^{-n}([\omega])\right) & =\sum_{\tau \in I^{n}} \mu([\tau \omega])=\sum_{\tau \in I^{n}} \int\left|\phi_{\tau \omega}^{\prime}\right|^{\delta} d m \geq \sum_{\tau \in I^{n}} K^{-\delta}\left\|\phi_{\tau}^{\prime}\right\|^{\delta} \int\left|\phi_{\omega}^{\prime}\right|^{\delta} d m \\
& =K^{-\delta} \int\left|\phi_{\omega}^{\prime}\right|^{\delta} d m \sum_{\tau \in I^{n}}\left\|\phi_{\tau}^{\prime}\right\|^{\delta} \geq K^{-\delta} \mu([\omega]) \mu\left(I^{\infty}\right)=K^{-\delta} \mu([\omega])
\end{aligned}
$$

and

$$
\begin{aligned}
\mu\left(\sigma^{-n}([\omega])\right) & =\sum_{\tau \in I^{n}} \mu([\tau \omega])=\sum_{\tau \in I^{n}} \int\left|\phi_{\tau \omega}^{\prime}\right|^{\delta} d m \\
& \leq \sum_{\tau \in I^{n}}\left\|\phi_{\tau}^{\prime}\right\|^{\delta} \int\left|\phi_{\omega}^{\prime}\right|^{\delta} d m=\int\left|\phi_{\omega}^{\prime}\right|^{\delta} d m \sum_{\tau \in I^{n}}\left\|\phi_{\tau}^{\prime}\right\|^{\delta} \\
& \leq K^{\delta} \mu([\omega]) .
\end{aligned}
$$

Therefore, $K^{-\delta} \mu([\omega]) \leq \mu^{*}([\omega]) \leq K^{\delta} \mu([\omega])$ and these inequalities extend to all Borel subsets of $I^{*}$. Thus, to complete the proof of our theorem we only need to show the ergodicity of $\mu^{*}$ or equivalently of $\mu$. Toward this end take a Borel set $A \in I^{\infty}$ with $\mu(A)>0$. Since the nested family of sets $\left\{[\tau]: \tau \in I^{*}\right\}$ generates the Borel $\sigma$-algebra on $I^{\infty}$, for every $n \geq 0$ and every $\omega \in I^{n}$ we can find a subfamily $Z$ of $I^{*}$ consisting of mutually incomparable words and such that $A \subset \bigcup\{[\tau]: \tau \in Z\}$ and $\sum_{\tau \in Z} \mu([\omega \tau]) \leq 2 \mu(\omega A)$, where 
$\omega A=\{\omega \rho: \rho \in A\}$. Then

$$
\begin{aligned}
\mu\left(\sigma^{-n}(A) \cap[\omega]\right) & =\mu(\omega A) \geq \frac{1}{2} \sum_{\tau \in Z} \mu([\omega \tau])=\frac{1}{2} \sum_{\tau \in Z} \int\left|\phi_{\omega \tau}^{\prime}\right|^{\delta} d m \\
& \geq \frac{1}{2} K^{-\delta}\left\|\phi_{\omega}^{\prime}\right\|^{\delta} \sum_{\tau \in Z} \int\left|\phi_{\tau}^{\prime}\right|^{\delta} d m \geq \frac{1}{2} K^{-\delta} \int\left|\phi_{\omega}^{\prime}\right|^{\delta} d m \sum_{\tau \in Z} \mu([\tau]) \\
& \geq \frac{1}{2} K^{-\delta} \mu([\omega]) \mu(\bigcup\{[\tau]: \tau \in Z\}) \geq \frac{1}{2} K^{-\delta} \mu(A) \mu([\omega]) .
\end{aligned}
$$

Therefore $\mu\left(\sigma^{-n}\left(I^{\infty} \backslash A\right) \cap[\omega]\right)=\mu\left([\omega] \backslash \sigma^{-n}(A) \cap[\omega]\right)=\mu([\omega])-\mu\left(\sigma^{-n}(A) \cap[\omega]\right) \leq$ $\left(1-\left(2 K^{\delta}\right)^{-1} \mu(A)\right) \mu([\omega])$. Hence for every Borel set $A \subset I^{\infty}$ with $\mu(A)<1$, for every $n \geq 0$, and for every $\omega \in I^{n}$ we get

$$
\mu\left(\sigma^{-n}(A) \cap[\omega]\right) \leq\left(1-\left(2 K^{\delta}\right)^{-1}(1-\mu(A))\right) \mu([\omega]) .
$$

In order to conclude the proof of ergodicity of $\sigma$ suppose that $\sigma^{-1}(A)=A$ and $0<$ $\mu(A)<1$. Put $\gamma=1-\left(2 K^{\delta}\right)^{-1}(1-\mu(A))$. Note that $0<\gamma<1$. In view of $(3.5)$, for every $\omega \in I^{*}$ we get $\mu(A \cap[\omega])=\mu\left(\sigma^{-|\omega|}(A) \cap[\omega]\right) \leq \gamma \mu([\omega])$. Take now $\eta>1$ so small that $\gamma \eta<1$ and choose a subfamily $R$ of $I^{*}$ consisting of mutually incomparable words and such that $A \subset \bigcup\{[\omega]: \omega \in R\}$ and $\mu(\bigcup\{[\omega]: \omega \in R\}) \leq \eta \mu(A)$. Then $\mu(A) \leq \sum_{\omega \in R} \mu(A \cap[\omega]) \leq \sum_{\omega \in R} \gamma \mu([\omega])=\gamma \mu(\bigcup\{[\omega]: \omega \in R\}) \leq \gamma \eta \mu(A)<\mu(A)$. This contradiction finishes the proof.

Theorem 3.9. There exists exactly one $\delta$-semiconformal measure.

Proof. Since $m$ is $\delta$-semiconformal we are only left to prove its uniqueness. So, let $m_{1}$ be another $\delta$-semiconformal measure and let $\mu_{1}$ be the probability measure produced in Lemma 3.6 applied to the measure $m_{1}$. Then for every $\omega \in I^{*}$ we have $K^{-\delta} \leq$ $\mu_{1}([\omega]) / \mu([\omega]) \leq K^{\delta}$, whence $\mu_{1}$ and $\mu$ are equivalent and the Radon-Nikodym derivative $\rho$ satisfies $K^{-\delta} \leq \rho \leq K^{\delta}$. We also have $\mu([\sigma(\omega)])=\int\left|\phi_{\sigma(\omega)}^{\prime}\right|^{\delta} d m$ and $\mu([\omega])=\int\left|\phi_{\omega}^{\prime}\right|^{\delta} d m=$ $\int\left|\phi_{\omega_{1}}^{\prime}\left(\phi_{\sigma(\omega)}(x)\right)\right|^{\delta}\left|\phi_{\sigma(\omega)}^{\prime}(x)\right|^{\delta} d m(x)$ and hence $\inf \left\{\left|\phi_{\omega_{1}}^{\prime}(x)\right|^{\delta}: x \in \phi_{\sigma(\omega)}(X)\right\} \mu([\sigma(\omega)]) \leq$ $\mu([\omega]) \leq \sup \left\{\left|\phi_{\omega_{1}}^{\prime}(x)\right|^{\delta}: x \in \phi_{\sigma(\omega)}(X)\right\} \mu([\sigma(\omega)])$. Since $\phi_{\omega_{1}}^{\prime}$ is a continuous function on $X$, we thus obtain that for every $\omega \in I^{\infty}$

$$
\lim _{n \rightarrow \infty} \frac{\mu\left(\left[\left.\omega\right|_{n}\right]\right)}{\mu\left(\left[\left.\sigma(\omega)\right|_{n-1}\right]\right)}=\left|\phi_{\omega_{1}}^{\prime}(\pi(\sigma(\omega)))\right|^{\delta}
$$

and the same formula is true with $\mu$ replaced by $\mu_{1}$. In view of Theorem 3.8 , there exists a set of points $\omega \in I^{\infty}$ with $\mu$ measure 1 for which the Radon-Nikodym derivatives $\rho(\omega)$ and $\rho(\sigma(\omega))$ both are defined. Let $\omega \in I^{\infty}$ be such a point. Then using (3.6) and its version for $\mu_{1}$, we obtain

$$
\begin{aligned}
\rho(\omega) & =\lim _{n \rightarrow \infty}\left(\frac{\mu_{1}\left(\left[\left.\omega\right|_{n}\right]\right)}{\mu\left(\left[\left.\omega\right|_{n}\right]\right)}\right)=\lim _{n \rightarrow \infty}\left(\frac{\mu_{1}\left(\left[\left.\omega\right|_{n}\right]\right)}{\mu_{1}\left(\left[\left.\sigma(\omega)\right|_{n-1}\right]\right)} \cdot \frac{\mu_{1}\left(\left[\left.\sigma(\omega)\right|_{n-1}\right]\right)}{\mu\left(\left[\left.\sigma(\omega)\right|_{n-1}\right]\right)} \cdot \frac{\mu\left(\left[\left.\sigma(\omega)\right|_{n-1}\right]\right)}{\mu\left(\left[\left.\omega\right|_{n}\right]\right)}\right) \\
& =\left|\phi_{\omega_{1}}^{\prime}(\pi(\sigma(\omega)))\right|^{\delta} \rho(\sigma(\omega))\left|\phi_{\omega_{1}}^{\prime}(\pi(\sigma(\omega)))\right|^{-\delta}=\rho(\sigma(\omega))
\end{aligned}
$$


But since, in view of Theorem 3.8, $\sigma$ is ergodic with respect to $\mu$, we conclude that $\rho$ is $\mu$-almost everywhere constant. Since $\mu_{1}$ and $\mu$ are both probabilistic, $\mu_{1}=\mu$. So, applying Lemma 3.7 finishes the proof.

Now, coming back to (OSC) and (2.7) we shall prove the existence (and uniqueness) of conformal measures. In fact we shall show that every measure fulfilling slightly weaker requirements than a semiconformal measure is conformal.

Lemma 3.10. A Borel probability measure $\nu$ on $X$ is $\delta$-conformal if and only if $P(\delta)=0$ and $\nu\left(\phi_{\omega}(A)\right) \geq \int_{A}\left|\phi_{\omega}^{\prime}\right|^{\delta} d \nu$ for all $\omega \in I^{*}$ and for all Borel subsets $A$ of $X$.

Proof. That conformal measures satisfy the requirements appearing in this lemma follows from their definition and Lemma 3.4. In order to prove the harder part first we shall show that condition (2.12) is satisfied, then that $\nu(J)=1$, and finally that $(2.11)$ holds. So, in order to prove (2.12) suppose to the contrary that $\nu\left(\phi_{\rho}(X) \cap \phi_{\tau}(X)\right)>0$ for some $q \geq 1$ and two distinct words $\rho, \tau \in I^{q}$. Let $E=\phi_{\rho}(X) \cap \phi_{\tau}(X)$ and for every $n \geq 1$ let $E_{n}=\bigcup_{\omega \in I^{n}} \phi_{\omega}(E)$. Since each element of $E_{n}$ admits at least two different codes of length $n+q$ which agree on the initial segment of length $n$, it follows from Lemma 2.6 that $\bigcap_{k=1}^{\infty} \bigcup_{n=k}^{\infty} E_{n}=\emptyset$. On the other hand by (3.3), (BDP), and Lemma 2.6 we get $\nu\left(E_{n}\right) \geq \beta \lambda_{d-1}^{-1}\left(S^{d-1}\right) K^{-\delta} \nu(E)$, thus $\nu\left(\bigcap_{k=1}^{\infty} \bigcup_{n=k}^{\infty} E_{n}\right) \geq \beta \lambda_{d-1}^{-1}\left(S^{d-1}\right) K^{-\delta} \nu(E)>0$. This contradiction shows that

$$
\nu\left(\phi_{\rho}(X) \cap \phi_{\tau}(X)\right)=0
$$

for all incomparable words $\rho, \tau \in I^{*}$. In order to show that $\nu(J)=1$ suppose to the contrary that $\nu(X \backslash J)>0$. In view of (3.7) for all $\omega \in I^{*}$ we have $\nu\left(\phi_{\omega}(X \backslash J) \cap J\right)=$ $\nu\left(\bigcup_{\tau \in I^{|\omega|}} \phi_{\omega}(X \backslash J) \cap \phi_{\tau}(J)\right) \leq \sum_{\tau \in I^{|\omega|}} \nu\left(\phi_{\omega}(X \backslash J) \cap \phi_{\tau}(J)\right)=0$. Hence setting $E_{n}=\bigcup_{\omega \in I^{n}} \phi_{\omega}(X \backslash J)$ we get $\nu\left(J \cap \bigcup_{n \geq 1} E_{n}\right)=0$. On the other hand $\nu\left(E_{n}\right) \geq$ $K^{-\delta} \nu(X \backslash J)$ (because of (3.7) we can skip the factor $\beta \lambda_{d-1}^{-1}\left(S^{d-1}\right)$ here) and therefore $\nu\left(\bigcap_{k=1}^{\infty} \bigcup_{n=k}^{\infty} E_{n}\right) \geq K^{-\delta} \nu(X \backslash J)>0$. Moreover

$$
\bigcap_{k=1}^{\infty} \bigcup_{n=k}^{\infty} E_{n} \subset \bigcap_{k=1}^{\infty}\left(\bigcup_{n=k}^{\infty} \bigcup_{\omega \in I^{n}} \phi_{\omega}(X)\right)=\bigcap_{k=1}^{\infty} \bigcup_{\omega \in I^{k}} \phi_{\omega}(X)=J .
$$

Combining the formulae occuring at the ends of the last three sentences we fall into a contradiction which proves that $\nu(J)=1$.

Now we need and we are in position to prove that the $\delta$-semiconformal measure $m$ is $\delta$-conformal. Indeed, $m$ satisfies all conditions placed in the right-hand side of Lemma 3.10. Moreover, using (3.7), (3.2), and Lemma 3.6, given an integer $n \geq 1$, we can write $1=$ $m(X)=m\left(\bigcup_{\omega \in I^{n}} \phi_{\omega}(X)\right)=\sum_{\omega \in I^{n}} m\left(\phi_{\omega}(X)\right) \geq \sum_{\omega \in I^{n}} \int\left|\phi_{\omega}^{\prime}\right|^{\delta} d m=1$. Therefore $m\left(\phi_{\omega}(X)\right)=\int\left|\phi_{\omega}^{\prime}\right|^{\delta} d m$ for all $\omega \in I^{n}$. Define now two finite measures $m_{1}$ and $m_{2}$ on $X$ in the following way: $m_{1}(A)=\int_{A}\left|\phi_{\omega}^{\prime}\right|^{\delta} d m$ and $m_{2}(A)=m\left(\phi_{\omega}(A)\right)$. Since we know that $m_{1}(X)=m_{2}(X)$ and $m_{1}(A) \leq m_{2}(A)$ for all Borel sets $A$, we conclude that $m_{1}=m_{2}$. Hence, conformality of $m$ is proven. 
Let us now return to the measure $\nu$. We shall show that $m$ is absolutely continuous with respect $\nu$. Indeed, it follows from conformality of $m$ and (BDP) that $K^{-\delta}\left\|\phi_{\omega}^{\prime}\right\|^{\delta} \leq$ $m\left(\phi_{\omega}(X)\right) \leq\left\|\phi_{\omega}^{\prime}\right\|^{\delta}$ for all $\omega \in I^{*}$. Since, by the assumptions, $\nu\left(\phi_{\omega}(X)\right) \geq K^{-\delta}\left\|\phi_{\omega}^{\prime}\right\|^{\delta}$, we therefore obtain $m\left(\phi_{\omega}(X)\right) \leq K^{\delta} \nu\left(\phi_{\omega}(X)\right)$. So, using (3.7), we conclude that $m$ is absolutely continuous with respect to $\nu$ and $\rho=d m / d \nu \leq K^{\delta} \nu$-a.e. Repeating essentially the argument from the proof of Theorem 3.9 to show that $\rho$ is almost everywhere constant, we proceed as follows. In view of Lemma 3.7 and Theorem 3.8 there exists a set of points $\omega \in I^{\infty}$ with $\mu$ measure 1 for which the Radon-Nikodym derivatives $\rho \circ \pi(\omega)$ and $\rho \circ \pi(\sigma(\omega))$ both are defined. Let $\omega \in I^{\infty}$ be such a point. Then

$$
\begin{aligned}
\rho \circ \pi(\omega) & =\lim _{n \rightarrow \infty}\left(\frac{m\left(\phi_{\left.\omega\right|_{n}}(X)\right)}{\nu\left(\phi_{\left.\omega\right|_{n}}(X)\right)}\right) \\
& =\lim _{n \rightarrow \infty}\left(\frac{m\left(\phi_{\left.\omega\right|_{n}}(X)\right)}{m\left(\phi_{\left.\sigma(\omega)\right|_{n-1}}(X)\right)} \cdot \frac{m\left(\phi_{\left.\sigma(\omega)\right|_{n-1}}(X)\right)}{\nu\left(\phi_{\left.\sigma(\omega)\right|_{n-1}}(X)\right)} \cdot \frac{\nu\left(\phi_{\left.\sigma(\omega)\right|_{n-1}}(X)\right)}{\nu\left(\phi_{\left.\omega\right|_{n}}(X)\right)}\right) \\
& \leq \lim _{n \rightarrow \infty}\left(\frac{\int_{\phi_{\left.\sigma(\omega)\right|_{n-1}}(X)}\left|\phi_{\omega}^{\prime}\right|^{\delta} d m}{m\left(\phi_{\left.\sigma(\omega)\right|_{n-1}}(X)\right)}\right) \cdot \rho(\pi(\sigma(\omega))) \cdot \lim _{n \rightarrow \infty}\left(\frac{\int_{\phi_{\left.\sigma(\omega)\right|_{n-1}}(X)}\left|\phi_{\omega}^{\prime}\right|^{\delta} d m}{m\left(\phi_{\left.\sigma(\omega)\right|_{n-1}}(X)\right)}\right) \\
& =\mid \phi_{\omega}^{\prime}\left(\left.\pi(\sigma(\omega))\right|^{\delta} \rho(\pi(\sigma(\omega))) \mid \phi_{\omega}^{\prime}\left(\left.\pi(\sigma(\omega))\right|^{-\delta}=\rho(\pi(\sigma(\omega)))\right.\right.
\end{aligned}
$$

So, by the Birkhoff ergodic theorem, $\rho \circ \pi(\omega)$ is $m$-a.e. constant and so is the RadonNikodym derivative $\rho: J \rightarrow[0, \infty)$. Keep the same symbol $\rho$ for this value. Since both measures $m$ and $\nu$ are probabilistic, $\rho \geq 1$. In the proof of the previous theorem we were done at this point concluding that $\rho=1$ since $\mu_{1}$ and $\mu_{2}$ were equivalent. Here an additional argument is needed. And indeed, if $\rho>1 \mathrm{~m}$-almost everywhere, define the set $Z=\{x \in J: \rho(x)=0\}$. Then $\nu(Z)=1-1 / \rho>0$. We claim that

$$
\nu\left((J \backslash Z) \cap \phi_{\omega}(Z)\right)=0
$$

for all $\omega \in I^{*}$. Indeed, if $\nu\left((J \backslash Z) \cap \phi_{\omega}(Z)\right)>0$ for some $\omega \in I^{*}$, then $m\left(\phi_{\omega}(Z)\right) \geq$ $m\left((J \backslash Z) \cap \phi_{\omega}(Z)\right)=\nu\left((J \backslash Z) \cap \phi_{\omega}(Z)\right) / \rho>0$ which by conformality of $m$ implies that $m(Z)>0$. This contradiction finishes the proof of (3.8). But now it follows from (3.8) that the probability measure $\left.\nu\right|_{Z} / \nu(Z)$ satisfies the assumptions of the right-hand side of Lemma 3.10, hence from what has been proved we conclude that $m$ is absolutely continuous with respect to $\left.\nu\right|_{Z} / \nu(Z)$. This however contradicts the definition of the set $Z$ and finishes the proof.

We would like to repeat here the following consequence of Lemma 2.6 which has been used in the proof of Lemma 3.10:

If $\rho$ and $\tau$ are two distinct words of the same length, $E=\phi_{\rho}(X) \cap \phi_{\tau}(X)$, and $E_{n}=\bigcup_{\omega \in I^{n}} \phi_{\omega}(E)$, then $\limsup _{n \rightarrow \infty} E_{n}=\emptyset$.

As an immediate consequence of Lemma 3.10, Theorem 3.5, and (3.2) we get the following. 
Corollary 3.11. Let $m$ be a $\delta$-semiconformal measure. Then $m$ is $\delta$-conformal. Also $m$-almost every point $x \in J$ has a unique representation in the form $x=\pi(\omega), \omega \in I^{\infty}$, i.e., the set $\pi^{-1}(x)$ is a singleton.

Remark 3.12. Notice that the measure $\mu^{*}$ pulls down canonically to the limit set $J$ giving rise to the measure $m^{*}=\mu^{*} \circ \pi^{-1}$ which is equivalent to $m$. Notice also that since $m$-almost every point $x \in J$ has a unique representation in the form $x=\pi(\omega), \omega \in I^{\infty}$, the formula $T(x)=\phi_{\omega_{1}}^{-1}(x)$ defines $\mu^{*}$-a.e. a measurable map on $J$. Observe that $T$ commutes with the shift map $\sigma$ on $I^{\infty}$ via the invertible map $\pi$ establishing a measurable isomorphism between the systems $\left(\sigma, \mu^{*}\right)$ on $I^{\infty}$ and $\left(T, m^{*}\right)$ on $J$. Frequently, in the sequel we will simply identify these two systems, especially writing $\sigma$ also for $T$. Let us also remark that in the appendix, we give a direct method of obtaining the measure $m^{*}$.

Let us now derive some geometric consequences of these results.

Lemma 3.13. A t-conformal measure exists if and only if $P(t)=0$.

Proof. The proof comes up as an immediate consequence of Lemma 3.4, Theorem 3.5 and Corollary 3.11.

For a c.i.f.s. $S$ denote by $h$ or by $h_{S}$, the Hausdorff dimension $\operatorname{HD}(J)$ of the corresponding limit set $J$. We call a c.i.f.s. $S$ regular if it admits a $t$-conformal measure, or, in view of Corollary 3.11 and Lemma 3.13, equivalently if the equation $\mathrm{P}(t)=0$ has a solution.

The following lemma has been proved in [2]. For the sake of completeness we prove it below giving a slightly different proof which fits better into our general approach.

Lemma 3.14. If $I$ is finite, then the system $\left\{S=\phi_{i}: i \in I\right\}$ is regular and there exists $C \geq 1$ such that

$$
C^{-1} \leq \frac{m(B(x, r))}{r^{\delta}} \leq C
$$

for all $x \in J$ and $0<2 r<\operatorname{diam}(X)$, where $P(\delta)=0$. In particular, $0<H_{\delta}(J), \Pi_{\delta}(J)<\infty$ and $\delta=h$.

Proof. Our system is regular since $0<\mathrm{P}(t)=\log \#(I)<\infty$. Since $I$ is finite, the number $\xi=\inf \left\{\left\|\phi_{i}^{\prime}\right\|: i \in I\right\}$ is positive. Consider $x=\pi(\omega), \omega \in I^{\infty}, 0<2 r<\operatorname{diam}(X)$, and let $n \geq 0$ be the smallest integer such that $\phi_{\left.\omega\right|_{n}}(X) \subset B(x, r)$. Then by (3.2) and (BDP), $m(B(x, r)) \geq K^{-\delta}\left\|\phi_{\left.\omega\right|_{n}}^{\prime}\right\|^{\delta}$. From the minimality of $n$ we conclude that $\phi_{\left.\omega\right|_{n-1}}(X)$ is not contained in $B(x, r)$. Thus, by (BDP) and (BDP.4), we get $r \leq \operatorname{diam}\left(\phi_{\left.\omega\right|_{n-1}}(X)\right) \leq$ $D\left\|\phi_{\left.\omega\right|_{n-1}}^{\prime}\right\| \leq D K\left\|\phi_{\omega_{n}}^{\prime}\right\|^{-1}\left\|\phi_{\left.\omega\right|_{n}}^{\prime}\right\| \leq D K \xi^{-1}\left\|\phi_{\left.\omega\right|_{n}}^{\prime}\right\|$. Therefore

$$
m(B(x, r)) \geq\left(D K^{2} \xi^{-1}\right)^{-\delta} r^{\delta}
$$


Thus by Theorem 2.8(1), $\mathrm{H}_{\delta}(J)<\infty$ and by Theorem 2.9(2), $\Pi_{\delta}(J)<\infty$. Now let $Z$ be the family of all minimal (in the sense of length) words $\omega \in I^{*}$ such that $\phi_{\omega}(X) \cap B(x, r) \neq \emptyset$ and $\phi_{\omega}(X) \subset B(x, 2 r)$. Then $\operatorname{diam}\left(\phi_{\left.\omega\right|_{|\omega|-1}}(X)\right) \geq r$. Let $R=\left\{\left.\omega\right|_{|\omega|-1}: \omega \in Z\right\}$. Note that $R$ is finite and therefore we can find a finite subfamily $R^{*}$ of $R$ consisting of mutually incomparable words such that each element of $R$ is an extension of an element from $R^{*}$. Temporarily fix an element $\tau \in R^{*}$ and take $i \in I$ such that $\tau i \in Z$. Then $4 r \geq$ $\operatorname{diam}\left(\phi_{\tau i}(X)\right) \geq D^{-1}\left\|\phi_{\tau i}^{\prime}\right\| \geq D^{-1} K^{-1}\left(\left\|\phi_{\tau}^{\prime}\right\|\right)\left(\left\|\phi_{i}^{\prime}\right\|\right) \geq D^{-2} K^{-1} \xi \operatorname{diam}\left(\phi_{\tau}(X)\right)$. Hence $\operatorname{diam}\left(\phi_{\tau}(X)\right) \leq 4 K D^{2} \xi^{-1} r$ and therefore $\bigcup_{\tau \in R^{*}} \phi_{\tau}(X) \subset B\left(x,\left(1+4 K D^{2} \xi^{-1}\right) r\right)$. On the other hand, as $r \leq \operatorname{diam}\left(\phi_{\tau}(X)\right) \leq D\left\|\phi_{\tau}^{\prime}\right\|$, it follows from (BDP.4) that $\left.\lambda\left(\phi_{\tau}(\operatorname{Int}(X))\right)\right) \geq$ $V_{d} D^{-d}\left\|\phi_{\tau}^{\prime}\right\|^{d} \geq V_{d} D^{-2 d} r^{d}$. Therefore $V_{d}\left(1+4 K D^{2} \xi^{-1}\right)^{d} r^{d}=\lambda\left(B\left(x,\left(1+4 K D^{2} \xi^{-1}\right) r\right)\right) \geq$ $\# R^{*} V_{d} D^{-2 d} r^{d}$, which implies that $\# R^{*} \leq D^{2 d}\left(1+4 K D^{2} \xi^{-1}\right)^{d}$ By the definition of $R^{*}$, we have $\pi^{-1}(B(x, r)) \subset \bigcup\left\{[\tau]: \tau \in R^{*}\right\}$. Therefore, since $\left\|\phi_{\tau}^{\prime}\right\| \leq 4 D K \xi^{-1} r$, using Lemma 3.7, we get

$$
\begin{aligned}
m(B(x, r)) & =\mu \circ \pi^{-1}(B(x, r)) \leq \sum_{\tau \in R^{*}} \mu([\tau]) \leq \sum_{\tau \in R^{*}}\left\|\phi_{\tau}^{\prime}\right\|^{\delta} \leq \sum_{\tau \in R^{*}}\left(4 D K \xi^{-1} r\right)^{\delta} \\
& \leq \# R^{*}\left(4 \xi^{-1} D K\right)^{\delta} r^{\delta} .
\end{aligned}
$$

So, applying Theorem 2.8(2) and Theorem 2.9(1) the proof is finished.

Let $\xi=\inf \{t \geq 0: \mathrm{P}(t)<0\}$ and let $\mathcal{F}$ in $(I)$ denote the family of all finite subsets of $I$. We shall prove a characterization of $\operatorname{HD}(J)$ which is well-known for finite systems and which goes along the line continued in [2], [3], [4], [15], [18], [17], and others.

Theorem 3.15. It holds $H D(J)=\xi=\sup \left\{h_{F}: F \in \mathcal{F}\right.$ in $\left.(I)\right\} \geq \theta$. If $P(t)=0$, then $t$ is the only zero of the function $P(t)$ and $t=H D(J)$.

Proof. Take $t>\xi$. Then, using (2.10), for every integer $n \geq 1$ sufficiently large we have $\sum_{\omega \in I^{n}} \operatorname{diam}\left(\phi_{\omega}(X)\right)^{t} \leq D^{t} \sum_{\omega \in I^{n}}\left\|\phi_{\omega}^{\prime}\right\|^{t} \leq D^{t} \exp (n \mathrm{P}(t) / 2)$. Since the family $\phi_{\omega}(X), \omega \in I^{n}$, is a cover of $J$ and since its diameters converge to 0 as $n \rightarrow \infty$, it follows from the estimate obtained that $\mathrm{H}_{t}(J)=0$. Thus $\operatorname{HD}(J) \leq \xi$. Set now $\eta=\sup \left\{h_{F}: F \in\right.$ $\mathcal{F}$ in $(I)\}$ and consider an arbitrary $t>\eta$. Then by Lemma 3.14 and (3.3) for every $n \geq 1$, we have

$$
\begin{aligned}
\sum_{\omega \in I^{n}}\left\|\phi_{\omega}^{\prime}\right\|^{t} & =\sup _{T \in \mathcal{F} i n(I)} \sum_{\omega \in T^{n}}\left\|\phi_{\omega}^{\prime}\right\|^{t} \leq \sup _{T}\left\{\sum_{\omega \in T^{n}}\left\|\phi_{\omega}^{\prime}\right\|^{h_{T}} s^{n\left(t-h_{T}\right)}\right\} \\
& \leq s^{(t-\eta) n} \sup _{T}\left\{\sum_{\omega \in T^{n}}\left\|\phi_{\omega}^{\prime}\right\|^{h_{T}}\right\} \leq s^{(t-\eta) n} \sup _{T} K^{h_{T}} \leq s^{(t-\eta) n} K^{\eta} .
\end{aligned}
$$

Hence $\mathrm{P}(t) \leq(t-\eta) \log s<0$ which gives $t \geq \xi$ and consequently $\eta \geq \xi$. Obviously $\eta \leq \operatorname{HD}(J)$, and since we have proved that $\operatorname{HD}(J) \leq \xi$, the proof of the "equality" part of the theorem is completed. The inequality $\theta \leq \xi$ follows immediately from definitions of both numbers. Finally, the last statement of the theorem is true since $\mathrm{P}(t)$ is continuous and strictly decreasing on $(\theta, \infty)$. 
The following theorem establishes also a continuity property of a conformal system with respect to its finite subsystems.

Theorem 3.16. If $S=\left\{\phi_{i}: i \in I\right\}$ is a regular system, then $\lim _{F \in \mathcal{F} \text { in }(I)} m_{F}=m_{I}$ in the weak* topology on $C(X)$.

Proof. Let $\nu$ be an arbitrary accumulation point (in the weak* topology on $C(X)$ ) of the sequence $\left\{m_{F}: F \in \mathcal{F} i n(I)\right\}$. We are to show that $\nu$ is $h=h_{I}$ conformal and in order to achieve this we will prove that the assumptions of Lemma 3.10 are satisfied. Indeed, $\mathrm{P}(h)=0$ since $S$ is regular. Therefore we can use (3.3), for example to conclude that for all $n \geq 1$

$$
\lim _{F \in \mathcal{F} i n(I)} \sum_{\omega \in F^{n}}\left|\phi_{\omega}^{\prime}\right|^{h}=\sum_{\omega \in I^{n}}\left|\phi_{\omega}^{\prime}\right|^{h}
$$

and the convergence is uniform with respect to $F \in \mathcal{F}$ in $(I)$. As, by Theorem $3.15, h=$ $\sup \left\{h_{F}: F \in \mathcal{F} i n(I)\right\}$, applying (3.1) we get that for every continuous function $f: X: \rightarrow$ $\mathbb{R}$, every $F \in \mathcal{F} \operatorname{in}(I)$, and every $n \geq 1$

$$
\int f d m_{F}=\int \sum_{\omega \in F^{n}}\left|\phi_{\omega}^{\prime}\right|^{h_{F}}\left(f \circ \phi_{\omega}\right) d m_{F} \geq \int \sum_{\omega \in F^{n}}\left|\phi_{\omega}^{\prime}\right|^{h}\left(f \circ \phi_{\omega}\right) d m_{F}
$$

Thus, using (3.9), continuity of $\sum_{\omega \in I^{n}}\left|\phi_{\omega}^{\prime}\right|^{h}$, and the definition of $\nu$ we obtain $\int f d \nu \geq$ $\int \sum_{\omega \in I^{n}}\left|\phi_{\omega}^{\prime}\right|^{h}\left(f \circ \phi_{\omega}\right) d \nu$. Now exactly as in the derivation of formula (3.2), we notice that this inequality extends to all bounded measurable functions $f: X \rightarrow \mathbb{R}$. In particular it is true with $f=1_{\phi_{\omega}(A)}$, where $\omega \in I^{*}$, and $A$ is any Borel subset of $X$. This gives $\nu\left(\phi_{\omega}(A)\right) \geq$ $\int_{A}\left|\phi_{\omega}^{\prime}\right|^{\delta} d \nu$ which finishes checking the assumptions of Lemma 3.10 and completes the proof.

The following immediate consequence of Theorem 3.15 has been proved, even in the random case, in [17].

Corollary 3.17. If $S$ is a linear c.i.f.s., then $H D(J)$ is the infimum of all $t \geq 0$ such that $\sum_{i \in I}\left\|\phi_{i}^{\prime}\right\|^{t}<1$.

Combining Lemma 3.13, Proposition 3.3, Theorem 3.9, and Theorem 3.15 we can collect the essence of our results so far proven in this section in the following way.

\section{Theorem 3.18.}

(a) If a t-semiconformal measure exists, then $t=h$.

(b) Each t-semiconformal measure is t-conformal.

(c) There exists at most one h-conformal (equivalently h-semiconformal) measure.

(d) The system $S$ is regular if and only if $P(h)=0$.

(e) The system $S$ is regular if and only if $P(t)=0$ for some positive $t$. 
Motivated by this theorem, from now on we will rather use the letter $h$ instead of $\delta$ even when referring to theorems including $\delta$ in their formulations explicitly. We call a subsystem $S^{\prime}=\left\{\phi_{i}: i \in I^{\prime}\right\}$ of the system $S=\left\{\phi_{i}: i \in I\right\}$ cofinite if $I^{\prime} \subset I$ and the difference $I \backslash I^{\prime}$ is finite. Dealing with cofinite subsystems we will need the following obvious lemma.

Lemma 3.19. The following conditions are equivalent.

(a) $\psi_{S}(t)<\infty$.

(b) There exists a cofinite subsystem $S^{\prime}$ of $S$ such that $\psi_{S^{\prime}}(t)<\infty$.

(c) For every cofinite subsystem $S^{\prime}$ of $S$ we have $\psi_{S^{\prime}}(t)<\infty$.

(d) $P_{S}(t)<\infty$.

(e) There exists a cofinite subsystem $S^{\prime}$ of $S$ such that $P_{S^{\prime}}(t)<\infty$.

(f) For every cofinite subsystem $S^{\prime}$ of $S$ we have $P_{S^{\prime}}(t)<\infty$.

In particular, $\theta_{S}=\theta_{S^{\prime}}$ for every cofinite subsystem $S^{\prime}$ of $S$.

We call a c.i.f.s. $S$ hereditarily regular if its every cofinite subsystem is regular.

Theorem 3.20. An infinite system $S$ is hereditarily regular if and only if $P(\theta)=$ $\infty \Leftrightarrow \psi(\theta)=\infty \Leftrightarrow\{t: P(t)<\infty\}=(\theta, \infty) \Leftrightarrow\{t: \psi(t)<\infty\}=(\theta, \infty)$. If $S$ is hereditarily regular, then $h>\theta$.

Proof. If $\{t: \mathrm{P}(t)<\infty\}=(\theta, \infty)$, then $S$ is hereditarily regular in view of Lemma 3.19, Theorem 3.18, and Proposition 3.3. If $\psi(\theta)<\infty$, then there exists a cofinite subsystem $S^{\prime}$ of $S$ such that $\psi_{S^{\prime}}(\theta)<1$, whence $\mathrm{P}_{S^{\prime}}(\theta)<0$. Therefore $S^{\prime}$ is not regular in view of Theorem 3.18, Theorem 3.15 and Proposition 3.3. All other equivalences involved in this theorem follow now from Lemma 3.19. Inequality $h>\theta$ follows now from Proposition 3.3 and Theorem 3.15 .

If $S$ is not regular we call it irregular. From Theorem 3.18, Theorem 3.15 and Proposition 3.3 we get the following.

Theorem 3.21. $S$ is irregular if and only if $P(h)<0 \Leftrightarrow P(\theta)<0$.

Theorem 3.22. If $S$ is irregular, then every cofinite subsystem $S^{\prime}$ of $S$ is irregular and $h_{S^{\prime}}=\theta_{S}$.

Proof. In view of Theorem $3.21 h_{S}=\theta_{S}$. In view of Lemma 3.19 and Theorem 3.21 $\mathrm{P}_{S^{\prime}}\left(\theta_{S^{\prime}}\right)=\mathrm{P}_{S^{\prime}}\left(\theta_{S}\right) \leq \mathrm{P}_{S}\left(\theta_{S}\right)<0$ and therefore it follows from Theorem 3.21 that $S^{\prime}$ is irregular. Thus, using Lemma $3.19, h_{S^{\prime}}=\theta_{S^{\prime}}=\theta_{S}$.

Theorem 3.23. $\lim _{T \in \mathcal{F} i n(I)} h_{I \backslash T}=\inf _{T \in \mathcal{F} i n(I)} h_{I \backslash T}=\theta_{S}$.

Proof. That $\lim _{T \in \mathcal{F} \text { in (I) }} h_{I \backslash T} \geq \theta_{S}$ follows from Theorem 3.15 and Lemma 3.19. In order to prove the opposite inequality fix $t>\theta_{S}$. Then $\psi_{S}(t)<\infty$, and therefore there exists $F \in \mathcal{F} i n(I)$ such that $\psi_{I \backslash T}(t)<1$ for every finite subset $T$ of $I$ containing $F$. Hence $\mathrm{P}_{I \backslash T}(t)<0$ for every finite subset $T$ of $I$ containing $F$ which shows that $\lim _{T \in \mathcal{F} i n(I)} h_{I \backslash T} \leq t$. The proof is finished. 
If the system $S$ is finite, then in view of Lemma 3.14, $m$ is equivalent to the $h_{\text {- }}$ dimensional Hausdorff measure on $J$. Since, as Example 5.5 shows (comp. also Example 5.7 and Theorem 6.4), this generally is no longer true for infinite systems, the natural question arises of whether at least the Hausdorff dimension of $m$ is equal to $h$. In order to give a sufficient condition, we find it appropriate, although not necessary, to use the notion of metric entropy to be found in [20] or [39] for example. We remark that since in our case the partition $\{[i]: i \in I\}$ generates the Borel $\sigma$-algebra on $I^{\infty}$, the metric entropy $\mathrm{h}_{\mu}(\sigma)$ of the system $\left(\sigma, \mu^{*}\right)$ is equal to $\inf _{k}\left\{\frac{-1}{k} \sum_{\omega \in I^{k}} \mu^{*}([\omega]) \log \left(\mu^{*}([\omega])\right)\right\}$ and note that this number is finite if and only if $\sum_{i \in I}-h \log \left(\left\|\phi_{i}^{\prime}\right\|\right)\left\|\phi_{i}^{\prime}\right\|^{h}$ is finite. We shall prove the following.

Theorem 3.24. If the metric dynamical system $\left(\sigma, \mu^{*}\right)$ has finite entropy, equivalently if the series $\sum_{i \in I}-h \log \left(\left\|\phi_{i}^{\prime}\right\|\right)\left\|\phi_{i}^{\prime}\right\|^{h}$ converges, then $H D(m)=h$.

Proof. Let $m(E)=1$. Since the series $\sum_{i \in I}-h \log \left(\left\|\phi_{i}^{\prime}\right\|\right)\left\|\phi_{i}^{\prime}\right\|^{h}$ converges, invoking (3.3), we conclude that the function $f: I^{\infty} \rightarrow \mathbb{R}, f(\omega)=h \log \left|\phi_{\omega_{1}}^{\prime} \pi((\sigma \omega))\right|$, is integrable with respect to the measure $\mu$, as well as, in view of Theorem 3.8, with respect to the invariant measure $\mu^{*}$. Let $F=\int f d \mu^{*},-\infty<F<0$. Fix $0<\gamma<1$. We will show $\operatorname{HD}(E) \geq \gamma h$. Take $q \geq 1$ so large and $\eta>0$ so small that $\frac{F+\eta}{F-\eta} \cdot \frac{q}{q+1}>\gamma$. Let now $J^{1}=\left\{x \in J: \# \pi^{-1}(x)=1\right\}$. By Remark 3.12, $m\left(J^{1}\right)=1$. For every $x \in J^{1}$ and every $n \geq 1$, set $\left.x\right|_{n}=\phi_{\left.\omega\right|_{n}}(x)$, where $\pi(\omega)=x$. In view of Birkhoff's ergodic theorem and Egorov's theorem there exists $J_{0} \subset J^{1} \cap E$ such that $m\left(J_{0}\right)>0$, and $\exp (n(F-\eta / 2)) \leq$ $\left|\phi_{\left.\omega\right|_{n}}^{\prime}\left(\sigma^{n}(x)\right)\right|^{n} \leq \exp (n(F+\eta / 2))$ for all $n \geq 1$ sufficiently large and all $x \in J_{0}$. Hence there exists $n_{0} \geq 1$ such that

$$
e^{n(F-\eta)} \leq m\left(\left.x\right|_{n}\right) \leq e^{n(F+\eta)},
$$

and

$$
\operatorname{diam}\left(\left.x\right|_{n}\right)^{h} \geq e^{n(F-\eta)}
$$

for all $x \in J_{0}$ and all $n \geq n_{0}$. Given now $0<r<\exp \left(n_{0}(F-\eta) / h\right)$ and $x \in J_{0}$, let $n(x, r) \geq 0$ be the minimal number $n$ such that $\operatorname{diam}\left(\left.x\right|_{n+1}\right)<r$. Using (3.10), we deduce that $n(x, r)+1>n_{0}$, hence $n(x, r) \geq n_{0}$ and $\operatorname{diam}\left(\left.x\right|_{n(x, r)}\right) \geq r$. In view of Lemma 2.7, for every $z \in J_{0}$ and every $0<r<\exp \left(n_{0}(F-\eta) / h\right)$, there are $k \leq V_{d} D^{2 d} \beta^{-1}\left(1+D^{-2}\right)$ points $x_{1}, \ldots, x_{k} \in J_{0}$ such that $\left.J_{0} \cap B(z, r) \subset \bigcup_{j=1}^{k} x_{j}\right|_{n\left(x_{j}, r\right)}$. Let $\tilde{m}=\left.m\right|_{J_{0}}$ be the measure $m$ restricted to the set $J_{0}$. Using (3.10) and (3.11) we get

$$
\begin{aligned}
\tilde{m}(B(z, r)) & \leq \sum_{j=1}^{k} m\left(\left.x_{j}\right|_{n\left(x_{j}, r\right)}\right) \leq \sum_{j=1}^{k} \mathrm{e}^{n\left(x_{j}, r\right)(F+\eta)} \\
& =\sum_{j=1}^{k} \exp \left(\left(n\left(x_{j}, r\right)+1\right)(F-\eta) \frac{F+\eta}{F-\eta} \cdot \frac{n\left(x_{j}, r\right)}{n\left(x_{j}, r\right)+1}\right) \leq \sum_{j=1}^{k}\left(\operatorname{diam}\left(\left.x_{j}\right|_{n\left(x_{j}, r\right)}\right)\right)^{h \gamma} \\
& \leq k r^{h \gamma} .
\end{aligned}
$$


Therefore, $\operatorname{HD}(E) \geq \gamma h$ and thus, $\operatorname{HD}(m) \geq \operatorname{HD}(\tilde{m}) \geq \gamma h$ which finishes the proof.

Corollary 3.25. If $S$ is hereditarily regular, then $H D(m)=h$.

Proof. Since $S$ is hereditarily regular there exists $\eta>0$ such that $\psi(h-\eta)<\infty$ which means that $\sum_{i \in I}\left\|\phi_{i}^{\prime}\right\|^{h-\eta}<\infty$. Since $\left\|\phi_{i}^{\prime}\right\|^{-\eta} \geq-h \log \left\|\phi_{i}^{\prime}\right\|$ for all but perhaps

finitely many $i \in I$, the series $\sum_{i \in I}-h \log \left(\left\|\phi_{i}^{\prime}\right\|\right)\left\|\phi_{i}^{\prime}\right\|^{h}$ converges. Thus Theorem 3.24 applies and the proof is finished.

We should note however that there are regular systems where the entropy of $\left(\sigma, \mu^{*}\right)$ is infinite and $\operatorname{HD}(m)=h$.

Let us finish this section with some comments which seem to be relevant in this place. Namely, for finite it c.i.f.s. $\operatorname{BD}(J)=\operatorname{HD}(J)$. This is

no longer the case for infinite c.i.f.s. Roughly speaking the reason is that $X(\infty)$ is in some sense highly independent of $J$. In particular, in Section 5 we give examples, even of regular locally finite linear systems, such that $\operatorname{HD}(J)<\operatorname{BD}(J)=\operatorname{HD}(\bar{J})=\operatorname{HD}(X(\infty))$.

\section{Geometric measures}

In this section our main objective is to study Lebesgue, Hausdorff, and packing measures of regular and hereditarily regular systems. In particular, we prove in this context some refinements of Theorems 2.8 and 2.9 relating the pointwise scaling behavior of conformal measure $m$ at the boundary, $X(\infty)$, to the values of Hausdorff and packing measures on the limit set $J$. Moreover, at the end of the section we prove a theorem which concerns irregular systems and establishes the "dimensionlessness" of their limit sets in the restricted sense. We begin with the following finer characterizations of the Hausdorff dimension of the limit sets which are more useful in estimating dimensions of various systems. Note that in condition (b) the distortion constant $K$ is involved whereas in condition (c) it is not $\left(c f\right.$., Section 6). Note also that $\underline{\psi}_{n}(t)$ treated as a function of $n$ with fixed $t$ is supermultiplicative whereas $\psi_{n}(t)$ was submultiplicative.

Theorem 4.1. If $S$ is a regular c.i.f.s., then for a real number $t \geq 0$ the following three conditions are equivalent.

(a) $t=h$ is the Hausdorff dimension of $J$.

(b) $t$ is the only number such that

$$
1 \leq \psi_{n}(t) \leq K^{d}
$$

for all $n \geq 1$, where $d$ is the dimension of the euclidean space containing $X$. 
(c) $t$ is the only number such that

$$
\underline{\psi}_{n}(t) \leq 1 \leq \psi_{n}(t)
$$

for all $n \geq 1$, where $\left.\underline{\psi}_{n}(t)\right)=\sum_{\omega \in I^{n}} \inf \left|\phi_{\omega}^{\prime}\right|^{t}$ and $\inf \left|\phi_{\omega}^{\prime}\right|=\inf \left\{\left|\phi_{\omega}^{\prime}(x)\right|: x \in X\right\}$.

Proof. That the Hausdorff dimension $h$ satisfies the inequalities appearing in (b) and (c) follows immediately from (3.3), Theorem 3.18, and since $\psi_{n}(t) \leq K^{t} \underline{\psi}_{n}(t) \leq \psi_{n}(t)$. Conversely, if $t$ satisfies either (b) or (c) for each $n \geq 1$, then

$$
\mathrm{P}(t)=\lim _{n \rightarrow \infty} \frac{1}{n} \log \psi_{n}(t)=\lim _{n \rightarrow \infty} \frac{1}{n} \log \underline{\psi}_{n}(t)=0
$$

and therefore $t=h$ in view of Theorem 3.15 .

Lemma 4.2. If $m$ is a t-conformal measure on $J$, then $H_{t}$ is absolutely continuous with respect to $m$ and $d H_{t} / d m \leq(D K)^{t}$. In particular, $H_{t}(J)$ is finite.

Proof. In view of (BDP),(BDP.1), and conformality of $m$ we have $\operatorname{diam}\left(\phi_{\omega}(J)\right) \leq$ $D\left\|\phi_{\omega}^{\prime}\right\|$ and $m\left(\phi_{\omega}(J)\right) \geq K^{-t}\left\|\phi_{\omega}^{\prime}\right\|^{t}$. Hence $\operatorname{diam}\left(\phi_{\omega}(J)\right)^{t} \leq(D K)^{t} m\left(\phi_{\omega}(J)\right)$. Let now $A$ be a closed subset of $J$ and for every $n \geq 1$ put $A_{n}=\left\{\omega \in I^{n}: \phi_{\omega}(J) \cap A \neq \emptyset\right\}$. Then the sequence of sets $\bigcup_{\omega \in A_{n}} \phi_{\omega}(J)$ is decreasing and $\bigcap_{n \geq 1}\left(\bigcup_{\omega \in A_{n}} \phi_{\omega}(J)\right)=A$. Therefore

$$
\begin{aligned}
\mathrm{H}_{t}(A) & \leq \liminf _{n \rightarrow \infty} \sum_{\omega \in A_{n}}\left(\operatorname{diam}\left(\phi_{\omega}(J)\right)\right)^{t} \leq \liminf _{n \rightarrow \infty}(D K)^{t} \sum_{\omega \in A_{n}} m\left(\phi_{\omega}(J)\right) \\
& =(D K)^{t} \liminf _{n \rightarrow \infty} m\left(\bigcup_{\omega \in A_{n}} \phi_{\omega}(J)\right)=(D K)^{t} m(A) .
\end{aligned}
$$

Since $J$ is a separable metric space, the measure $m$ is regular and therefore the inequality $\mathrm{H}_{t}(A) \leq(D K)^{t} m(A)$ extends to all Borel subsets of $J$. The proof is finished.

Let us now prove an analogous result for packing measures.

Lemma 4.3. If $m$ is a $t$-conformal measure for a c.i.f.s. $S=\left\{\phi_{i}: i \in I\right\}$ and either $I$ is finite or $J \cap \operatorname{Int}(X) \neq \emptyset$, then $m$ is absolutely continuous with respect to $\Pi_{t}$. Moreover the Radon-Nikodym derivative $d m / d \Pi_{t}$ is uniformly bounded away from infinity. In particular, $\Pi_{h}(J)>0$.

Proof. If $I$ is finite, then the result follows from Lemma 3.14. So, suppose that $J \cap \operatorname{Int}(X) \neq \emptyset$. Then there exists $q \geq 1$ and $\tau \in I^{q}$ such that $\phi_{\tau}(X) \subset \operatorname{Int}(X)$. Set $\gamma=\operatorname{dist}\left(\phi_{\tau}(X), \partial X\right)$. Let

$$
R=\left\{\omega \in I^{\infty}:\left.\omega\right|_{[n+1, n+q]}=\tau \text { for infinitely many } n ' s\right\}
$$

and let $R_{0}$ be the set of those elements of $I^{\infty}$ which contain no subword $\tau$. Since $[\tau] \cap R_{0}=$ $\emptyset$, we get $\mu\left(R_{0}\right)<1$, and since $\sigma^{-1}\left(I^{\infty} \backslash R_{0}\right) \subset I^{\infty} \backslash R_{0}$, it follows from ergodicity of $\sigma$ proven in Theorem 3.8 that $\mu\left(R_{0}\right)=0$. As $I^{\infty} \backslash R=\bigcup_{n \geq 0} \sigma^{-n}\left(R_{0}\right)$, we obtain $\mu\left(I^{\infty} \backslash R\right)=0$. Therefore, using Lemma 3.7, we get $m(J \backslash \pi(R))=\mu \circ \pi^{-1}(J \backslash \pi(R)) \leq \mu\left(I^{\infty} \backslash R\right)=0$. 
Take now $\omega \in R$ and an integer $n \geq 1$ such that $\left.\omega\right|_{[n+1, n+q]}=\tau$. Put $x=$ $\pi(\omega)$ and consider the ball $B\left(x, K^{-1}\left\|\phi_{\left.\omega\right|_{n}}^{\prime}\right\| \gamma\right)$. Since by (BDP.3) $B\left(x, K^{-1}\left\|\phi_{\left.\omega\right|_{n}}^{\prime}\right\| \gamma\right) \subset$ $\phi_{\omega \mid}\left(B\left(\pi\left(\sigma^{n}(\omega)\right), \gamma\right)\right)$ and since $B\left(\pi\left(\sigma^{n}(\omega)\right), \gamma\right) \subset \operatorname{Int}(X) \subset X$, using (BDP.1) and conformality of $m$ we get

$$
\begin{aligned}
m\left(B\left(x, K^{-1}\left\|\phi_{\left.\omega\right|_{n}}^{\prime}\right\| \gamma\right)\right) & \leq\left\|\phi_{\left.\omega\right|_{n}}^{\prime}\right\|^{t} m\left(B\left(\pi\left(\sigma^{n}(\omega)\right), \gamma\right)\right) \leq\left\|\phi_{\left.\omega\right|_{n}}^{\prime}\right\|^{t} \\
& =\left(K \gamma^{-1}\right)^{t}\left(K^{-1}\left\|\phi_{\left.\omega\right|_{n}}^{\prime}\right\| \gamma\right)^{t} .
\end{aligned}
$$

Since $m(J \backslash \pi(R))=0$, applying Theorem 2.9(1) we thus get $\Pi_{t}(E) \geq\left(K \gamma^{-1}\right)^{t} b(d) m(E)$ for every Borel subset $E$ of $J$. The proof is finished.

The assumption $J \cap \operatorname{Int}(X) \neq \emptyset$ is actually known in the literature as the Strong Open Set Condition (SOSC). Actually, the (SOSC) requires the existence of a set $X$ satisfying (2.6) - (2.7) and the condition $J \cap \operatorname{Int}(X) \neq \emptyset$. In order to clarify the situation note that $X$ is not uniquely determined by the contractions $\phi_{i}$ and the limit set $J$. So the question is: Given a c.i.f.s., can one adjust $X$ so that (SOSC) holds? This is exactly what Schief accomplishes in the case of a finite system of similarities (see [28]).

Now for each $n \geq 0$ put

$$
X_{n}=\bigcup_{\omega \in I^{n}} \phi_{\omega}(X) .
$$

We shall now prove two results concerning the $d$-dimensional Lebesgue measure $\lambda$ of these sets, the Lebesgue measure of the limit set $J$, and an estimate on the Hausdorff dimension of $J$.

Proposition 4.4. If $S$ is a c.i.f.s. and $\lambda_{d}\left(\operatorname{Int}(X) \backslash X_{1}\right)>0$, then there exists $0<\gamma<1$ such that $\lambda_{d}\left(X_{n}\right) \leq \gamma^{n} \lambda_{d}(X)$ for all $n \geq 1$. In particular, $\lambda_{d}(J)=0$.

Proof. Put $G=\operatorname{Int}(X) \backslash X_{1}$ and $\xi=K^{-d} \lambda_{d}(G) / \lambda_{d}(X)<1$. In view of (BDP) we have $\lambda_{d}\left(\phi_{\omega}(G)\right) \geq \xi \lambda_{d}\left(\phi_{\omega}(X)\right)$. In view of (OSC) we have $\phi_{\omega}(G) \cap \phi_{\tau}(X)=\emptyset$ if $\omega \neq \tau$ and $|\omega|=|\tau|$. Thus for all $n \geq 0$

$$
X_{n+1}=\bigcup_{\omega \in I^{n}} \phi_{\omega}\left(X_{1}\right) \subset \bigcup_{\omega \in I^{n}} \phi_{\omega}(X \backslash G)=\bigcup_{\omega \in I^{n}} \phi_{\omega}(X) \backslash \bigcup_{\omega \in I^{n}} \phi_{\omega}(G)=X_{n} \backslash \bigcup_{\omega \in I^{n}} \phi_{\omega}(G) .
$$

Therefore

$$
\begin{aligned}
\lambda_{d}\left(X_{n+1}\right) & \leq \lambda_{d}\left(X_{n}\right)-\lambda\left(\bigcup_{\omega \in I^{n}} \phi_{\omega}(G)\right)=\lambda_{d}\left(X_{n}\right)-\sum_{\omega \in I^{n}} \lambda_{d}\left(\phi_{\omega}(G)\right) \\
& \leq \lambda_{d}\left(X_{n}\right)-\xi \sum_{\omega \in I^{n}} \lambda_{d}\left(\phi_{\omega}(X)\right) \leq \lambda_{d}\left(X_{n}\right)-\xi \lambda_{d}\left(X_{n}\right)=(1-\xi) \lambda_{d}\left(X_{n}\right) .
\end{aligned}
$$

So, putting $\gamma=1-\xi$ finishes the proof.

Theorem 4.5. If $S$ is a regular c.i.f.s. and $\lambda_{d}\left(\operatorname{Int}(X) \backslash X_{1}\right)>0$, then $h=H D(J)<$ d. If conversely $\lambda_{d}\left(X \backslash X_{1}\right)=0$, then $S$ is regular, $\lambda_{d}(J)=\lambda_{d}(X)>0$, in particular, $H D(J)=d$, and $\lambda_{d} / \lambda_{d}(X)$ is the only conformal measure. 
Proof. In order to prove the first part suppose to the contrary that $h=d$. Then for every $\omega \in I^{*}$ and every Borel set $A \subset X$ with $\lambda_{d}(A)>0$ we have

$$
m\left(\phi_{\omega}(A)\right) \leq\left\|\phi_{\omega}^{\prime}\right\|^{d} m(A)=K^{-d}\left\|\phi_{\omega}^{\prime}\right\|^{d} \lambda_{d}(A) \frac{m(A)}{\lambda_{d}(A)} K^{d} \leq \lambda_{d}\left(\phi_{\omega}(A)\right) K^{d} \frac{m(A)}{\lambda_{d}(A)} .
$$

For every $n \geq 1$ and every $\omega \in I^{n}$ define $Y_{\omega}=\phi_{\omega}(X) \cap \bigcup_{\tau \in I^{n} \backslash\{\omega\}} \phi_{\tau}(X)$. Then the sets $\phi_{\omega}(X) \backslash Y_{\omega}, \omega \in I^{n}$, are mutually disjoint, in view of (2.14) $m\left(Y_{\omega}\right)=0$ for all $\omega \in I^{n}$, and $\phi_{\omega}^{-1}\left(Y_{\omega}\right) \subset \partial X$ by (OSC). Therefore, using (4.1), we get the following estimate

$$
\begin{aligned}
m\left(X_{n}\right) & =\sum_{\omega \in I^{n}} m\left(\phi_{\omega}(X) \backslash Y_{\omega}\right)=\sum_{\omega \in I^{n}} m\left(\phi_{\omega}\left(X \backslash \phi_{\omega}^{-1}\left(Y_{\omega}\right)\right)\right) \\
& \leq \sum_{\omega \in I^{n}} \lambda_{d}\left(\phi_{\omega}\left(X \backslash \phi_{\omega}^{-1}\left(Y_{\omega}\right)\right)\right) K^{d} \frac{m\left(X \backslash \phi_{\omega}^{-1}\left(Y_{\omega}\right)\right)}{\lambda_{d}\left(X \backslash \phi_{\omega}^{-1}\left(Y_{\omega}\right)\right)} \\
& \leq K^{d} \frac{m(X)}{\lambda_{d}(\operatorname{Int}(X))} \sum_{\omega \in I^{n}} \lambda_{d}\left(\phi_{\omega}(X) \backslash Y_{\omega}\right) \leq \frac{K^{d}}{\lambda_{d}(\operatorname{Int}(X))} \lambda_{d}\left(X_{n}\right) .
\end{aligned}
$$

Thus, by Proposition 4.4, $m(J)=\lim _{n \rightarrow \infty} m\left(X_{n}\right)=0$. This completes the proof of the first part.

Moving to the other part of the theorem notice first that for every $n \geq 0$ we have

$$
X_{n} \backslash X_{n+1}=\bigcup_{\omega \in I^{n}} \phi_{\omega}(X) \backslash \bigcup_{\omega \in I^{n}} \phi_{\omega}\left(X_{1}\right) \subset \bigcup_{\omega \in I^{n}}\left(\phi_{\omega}(X) \backslash \phi_{\omega}\left(X_{1}\right)\right)=\bigcup_{\omega \in I^{n}} \phi_{\omega}\left(X \backslash X_{1}\right) .
$$

Since $\lambda_{d}\left(X \backslash X_{1}\right)=0$, we therefore obtain $\lambda_{d}\left(X_{n} \backslash X_{n+1}\right)=0$ or equivalently $\lambda_{d}\left(X_{n}\right)=$ $\lambda_{d}\left(X_{n+1}\right)$. Hence $\lambda_{d}(J)=\lim _{n \rightarrow \infty} \lambda_{d}\left(X_{n}\right)=\lambda_{d}(X)>0$. In particular $h=d$. Now, it follows from Lemma 2.5 that $d \in F(S)$ and $\mathrm{P}(d) \leq 0$. And since $\psi_{n}(d) \geq \lambda_{d}(X)$ for all $n \geq$ 0 , we conclude that $\mathrm{P}(d)=0$, thus $S$ is regular. Since obviously $\lambda_{d}\left(\phi_{\omega}(A)\right)=\int_{A}\left|\phi_{\omega}^{\prime}\right|^{d} d \lambda_{d}$ for all $n \geq 0$ and all Borel subsets $A$ of $X$, all the assumptions of Lemma 3.10 are satisfied and therefore $\lambda_{d} / \lambda_{d}(X)$ is $d$-conformal. So, applying Theorem 3.18 finishes the proof.

Remark 4.6 As Example 5.3 shows Theorem 4.5 fails to be true if regularity of the system $S$ is relaxed, that is if $S$ is irregular, although still, as Proposition 4.4 says, $\lambda_{d}(J)=0$ and the Lebesgue measure of the sets $X_{n}$ decreases to 0 exponentially fast.

We would also like to point out that positivity of the Lebesgue measure of $\operatorname{Int}(X) \backslash X_{1}$, the assumption of both Proposition 4.4 and Theorem 4.5 is obviously satisfied if the interior of the set $\operatorname{Int}(X) \backslash X_{1}$ is nonempty.

Theorem 4.7. Suppose that $S=\left\{\phi_{i}: i \in I\right\}$ is a regular c.i.f.s. Then the following conditions are equivalent.

(a) $S$ is regular and $h_{S}>\theta_{S}$.

(b) There exists a proper cofinite subsystem $S^{\prime}$ of $S$ such that $h_{S^{\prime}}<h_{S}$.

(c) For every proper subsystem $S^{\prime}$ of $S$ we have $h_{S^{\prime}}<h_{S}$. 
Proof. That $(c) \Rightarrow(b)$ is obvious. The implication $(b) \Rightarrow(a)$ follows from Theorem 3.22 and Theorem 3.23. Thus, we are only left to show that $(a) \Rightarrow(c)$ and we only need to do it for cofinite subsystems. So, consider a proper subsystem $S^{\prime}=\left\{\phi_{i}: i \in I\right\}$ of $S$. Suppose first that $S^{\prime}$ is irregular and fix any number $\alpha \in\left(\theta_{S}, h_{S}\right)$. By Lemma 3.19 $\mathrm{P}_{S^{\prime \prime}}(\alpha)<\infty$ and therefore by Theorem 3.15, $h_{S^{\prime}} \leq \alpha<h_{S}$ and we are done in this case. So suppose that $S^{\prime}$ is regular and additionally suppose to the contrary that $h=h_{S^{\prime}}=h_{S}$. Let $m$ and $m^{\prime}$ be $h$-semiconformal measures associated respectively to the systems $S$ and $S^{\prime}$ and let $\mu$ and $\mu^{\prime}$ be corresponding probability measures on $I^{\infty}$ and $I^{\prime \infty}$ produced by Lemma 3.6. In view of (BDP), for every $\omega \in I^{* *}$ we have $\int\left|\phi_{\omega}^{\prime}\right|^{h} d m \leq\left\|\phi_{\omega}^{\prime}\right\|^{h}$ and $\int\left|\phi_{\omega}^{\prime}\right|^{h} d m \geq K^{-h}\left\|\phi_{\omega}^{\prime}\right\|^{h}$, and consequently $\mu^{\prime}([\omega]) \leq K^{h} \mu([\omega])$. Notice now that $I^{\prime \infty} \subset I^{\infty}$ and $\mu^{\prime}$ can be regarded as a probability measure on $I^{\infty}$. Then this last inequality enlarged by the formula $\mu^{\prime}([\omega])=0$ for $\omega \notin I^{\prime *}$ implies that $\mu^{\prime}$ is absolutely continuous with respect $\mu$ (even $d \mu^{\prime} / d \mu \leq K^{h}$ ). Thus it follows from Theorem 3.8 that $\mu^{\prime *}$ is absolutely continuous with respect to $\mu$. Since moreover, by Theorem 3.8, both measures $\mu^{*}$ and $\mu^{\prime *}$ are ergodic and $\sigma$-invariant on $I^{\infty}$ (the reader is encouraged to make himself certain about it for $\mu^{\prime}$ ), they must coincide. This however is a contradiction as $\mu^{\prime *}([k]) \leq K^{\delta} \mu^{\prime}([k])=0$ and $\mu^{*}([k]) \geq K^{-\delta} \mu([k])>0$. The proof is finished.

In view of Theorem $3.20, h_{S}>\theta_{S}$ for every hereditarily regular system $S$. Therefore, as a consequence of Theorem 4.7 we get the following.

Corollary 4.8. it If $S=\left\{\phi_{i}: i \in I\right\}$ is a hereditarily regular c.i.f.s. and $S^{\prime}$ is a proper subsystem of $S$, then $h_{S^{\prime}}<h_{S}$.

Lemma 4.9. If $S=\left\{\phi_{i}: i \in I\right\}$ is a regular c.i.f.s. and there exist a sequence of points $z_{j} \in X(\infty)$ and a sequence of positive reals $\left\{r_{j}: j \geq 1\right\}$ such that

$$
\limsup _{j \rightarrow \infty} \frac{m\left(B\left(z_{j}, r_{j}\right)\right)}{r_{j}^{h}}=\infty,
$$

then $H_{h}(J)=0$.

Proof. Fix $\varepsilon>0$ and take $z=z_{j}, r=r_{j}<\operatorname{dist}(X, \partial V)$ such that $m(B(z, r)) r^{-h} \geq$ $\varepsilon^{-1}$. Consider $J_{\infty}$ to be the subset of those points $x$ in $J$ that can be expressed as $x=\pi(\omega)$, where each element of $I$ appears in $\omega$ infinitely often. Since $z \in X(\infty)$ and by (2.5), there exists $i \in I$ such that $\phi_{i}(X) \subset B(z, r)$. Consider an index $k \geq 1$ such that $\omega_{k}=i$ and denote by $\psi$ the map $\phi_{\left.\omega\right|_{k-1}}$. By (BDP.1) $B\left(\psi(z),\left\|\psi^{\prime}\right\| r\right) \supset \psi(B(z, r))$, in particular $\operatorname{dist}(x, \psi(z)) \leq\left\|\psi^{\prime}\right\| r$. Consequently $B\left(x, 2\left\|\psi^{\prime}\right\| r\right) \supset \psi(B(z, r))$. Thus, by semiconformality of $m,(\mathrm{BDP})$, and the choice of $\varepsilon$ we get

$$
\begin{aligned}
m\left(B\left(x, 2\left\|\psi^{\prime}\right\| r\right)\right) & \geq K^{-h}\left\|\psi^{\prime}\right\|^{h} m(B(z, r)) \geq \varepsilon^{-1} K^{-h}\left\|\psi^{\prime}\right\|^{h} r^{h} \\
& =\varepsilon^{-1}(2 K)^{-h}\left(2\left\|\psi^{\prime}\right\| r\right)^{h}
\end{aligned}
$$

Hence, applying Theorem 2.8(1) and then letting $\varepsilon \rightarrow 0$ we get $\mathrm{H}_{h}\left(J_{\infty}\right)=0$. The same argument as in the proof of Lemma 4.3, based on ergodicity of $\sigma$ proven in Theorem 3.8, 
gives $m\left(J \backslash J_{\infty}\right)=0$ and therefore by Lemma $4.2, \mathrm{H}_{h}\left(J \backslash J_{\infty}\right)=0$ which completes the proof.

Lemma 4.10. Let $S=\left\{\phi_{i}: i \in I\right\}$ be a regular c.i.f.s. Suppose that there are two constants $L>0, \xi>0$, and $\gamma \geq 1$ such that for every $i \in I$ and every $r$ with $\gamma \operatorname{diam}\left(\phi_{i}(X)\right) \leq r \leq \xi$ there exists $y \in \bar{\phi}_{i}(V)$ such that $m(B(y, r)) \geq L r^{h}$. Then $\Pi_{h}(J)<$ $+\infty$.

Proof. First notice that by increasing $L$ if necessary, the assumption of the lemma continues to be fulfilled if the number $\xi$ is replaced by any other positive number. We take $\xi=\eta / 2$, where $\eta=\operatorname{dist}(X, \partial V)$. We can also assume $D^{-3} \eta<1$. Fix $0<r<\xi$, $x=\pi(\omega) \in J$, and take maximal $k \geq 0$ such that

$$
\phi_{\left.\omega\right|_{k}}(V) \supset B\left(x, D^{-2} r\right) .
$$

Abbreviate $\phi_{\left.\omega\right|_{k+1}}$ by $\psi$. Then $\psi(V)$ does not contain $B\left(x, D^{-2} r\right)$ and, as by (BDP.4), $\psi(V) \supset B\left(x, D^{-1}\left\|\psi^{\prime}\right\|\right)$, we find $D^{-2} r>D^{-1}\left\|\psi^{\prime}\right\|$. Hence, by (BDP.2), $B(x, r) \supset$ $B\left(x, D\left\|\psi^{\prime}\right\|\right) \supset \psi(V)$ and therefore, using semiconformality of $m$ we get $m(B(x, r)) \geq$ $K^{-h}\left\|\psi^{\prime}\right\|^{h} m(V)=K^{-h}\left\|\psi^{\prime}\right\|^{h}$. If now $\gamma D K\left\|\psi^{\prime}\right\| \geq\left(2 D^{3}\right)^{-1} \eta r$, then

$$
\frac{m(B(x, r))}{r^{h}} \geq \eta^{h}\left(2 D^{4} K^{2} \gamma\right)^{-h} .
$$

Otherwise,

$$
\gamma D K\left\|\psi^{\prime}\right\|<\left(2 D^{3}\right)^{-1} \eta r
$$

Set now $g=\phi_{\omega_{k+1}}$ and let $y$ be an arbitrary point in $g(V)$. Since $\operatorname{diam}(g(V)) \leq D\left\|g^{\prime}\right\|$ and since $\gamma \geq 1$, it follows from (4.3) that

$$
B\left(y,\left(2 D^{3}\right)^{-1} \eta\left\|\phi_{\left.\omega\right|_{k}}^{\prime}\right\|^{-1} r\right) \subset B\left(\pi\left(\sigma^{k}(\omega)\right), D^{-3} \eta\left\|\phi_{\left.\omega\right|_{k}}^{\prime}\right\|^{-1} r\right) .
$$

From (BDP.1)

$$
\begin{aligned}
\phi_{\left.\omega\right|_{k}}\left(B\left(\pi\left(\sigma^{k}(\omega)\right), D^{-3} \eta\left\|\phi_{\left.\omega\right|_{k}}^{\prime}\right\|^{-1} r\right)\right. & \subset B\left(x, D^{-3} \eta r\right) \\
& \subset B(x, r) .
\end{aligned}
$$

In view of (4.3), we have $\left(2 D^{3}\right)^{-1} \eta\left\|\phi_{\left.\omega\right|_{k}}^{\prime}\right\|^{-1} r \geq \gamma \operatorname{diam}(g(V))$. By (4.2) and (BDP.2), $D^{-3}\left\|\phi_{\left.\omega\right|_{k}}^{\prime}\right\|^{-1} r \leq 1$, hence $\left(2 D^{3}\right)^{-1} \eta\left\|\phi_{\left.\omega\right|_{k}}^{\prime}\right\|^{-1} r \leq \eta / 2$. As the number $\left(2 D^{3}\right)^{-1} \eta\left\|\phi_{\left.\omega\right|_{k}}^{\prime}\right\|^{-1} r$ does not depend on the choice of $y \in g(V)$, we can assume that $y$ satisfies the assumption of our lemma. Using this assumption, it follows from (4.4) and (4.5) that

$$
m(B(x, r)) \geq K^{-h}\left\|\phi_{\left.\omega\right|_{k}}^{\prime}\right\|^{h} L\left(2 D^{3}\right)^{-h} \eta^{h}\left\|\phi_{\left.\omega\right|_{k}}^{\prime}\right\|^{-h} r^{h}=L \eta^{h}\left(2 D^{3} K\right)^{-h} r^{h} .
$$

The proof is finished.

Lemma 4.11. Let $S=\left\{\phi_{i}: i \in I\right\}$ be a regular c.i.f.s. Suppose that there are two constants $L>0, \gamma \geq 1$ such that for every $i \in I$ and every $r \geq \gamma \operatorname{diam}\left(\phi_{i}(X)\right)$ there exists $y \in \phi_{i}(V)$ such that $m(B(y, r)) \leq L r^{h}$. Then $H_{h}(J)>0$. 
Proof. Set $\eta=\operatorname{dist}(X, \partial V)$. Without loss of generality, we can assume that $\gamma \operatorname{diam}(X)$ $\geq 1$. Take an arbitrary $x \in J$ and radius $r>0$. Set $\tilde{r}=2 K D \eta^{-1} r$. For every $z \in$ $B(x, r) \cap J$ consider a shortest word $\omega=\omega(z)$ such that $z \in \pi([\omega])$ and $\phi_{\omega}(X) \subset B(z, \tilde{r})$. Then $\operatorname{diam}\left(\phi_{\left.\omega\right|_{|\omega|-1}}(X)\right) \geq \tilde{r}$. Let $R=\left\{\left.\omega(z)\right|_{|\omega(z)|-1}: z \in J \cap B(x, r)\right\}$. Notice that $R$ is finite since $\lim _{i \in I} \operatorname{diam}\left(\phi_{i}(X)\right)=0$ and since $\lim _{n \rightarrow \infty} \sup \left\{\operatorname{diam}\left(\phi_{\omega}(X)\right): \omega \in I^{n}\right\}=0$. Therefore we can find a finite set $\left\{z_{1}, z_{2}, \ldots, z_{k}\right\} \subset J \cap B(x, r)$ such that the family $R^{*}=\left\{\left.\omega\left(z_{j}\right)\right|_{\left|\omega\left(z_{j}\right)\right|-1}: j=1, \ldots, k\right\} \subset R$ consists of mutually incomparable words and the family $\left\{\pi\left(\left.\omega\left(z_{j}\right)\right|_{\left|\omega\left(z_{j}\right)\right|-1}: j=1, \ldots, k\right\}\right.$ covers $B(x, r) \cap J$. Now, temporarily fix an element $z \in\left\{z_{1}, z_{2}, \ldots, z_{k}\right\}$, set $\omega=\omega(z), q=|\omega|$, and $\psi=\phi_{\left.\omega\right|_{q-1}}$. Since $\operatorname{diam}(\psi(\operatorname{Int}(X))) \geq \tilde{r}$, it follows from (2.10) that $\lambda_{d}(\psi(\operatorname{Int}(X)) \cap B(z, \tilde{r})) \geq \beta V_{d} D^{-2 D} r^{d}$. Therefore $2^{d} V_{d} \tilde{r}^{d}=$ $\lambda_{d}(B(x, 2 \tilde{r})) \geq \# R^{*} \beta V_{d} D^{-2 D} \tilde{r}^{d}$, which implies that $\# R^{*} \leq\left(2 D^{2}\right)^{d} \beta^{-1}$. By the choice of $\omega$ we have $D^{-1} K^{-1}\left\|\psi^{\prime}\right\| \cdot\left\|\phi_{\omega_{q}}^{\prime}\right\| \leq 2 \tilde{r}$, whence $2 K D^{2}\left\|\psi^{\prime}\right\|^{-1} \tilde{r} \geq D\left\|\phi_{\omega_{q}}^{\prime}\right\| \geq \operatorname{diam}\left(\phi_{\omega_{q}}(X)\right)$. So, if $y \in \phi_{\omega_{q}}(X)$ is the point from the assumptions of the lemma corresponding to the radius $4 \gamma K D^{2}\left\|\psi^{\prime}\right\|^{-1} \tilde{r} \geq 2 \gamma \operatorname{diam}\left(\phi_{\omega_{q}}(X)\right)$, using (BDP.3) and inequality $2 r K\left\|\psi^{\prime}\right\|^{-1} \leq$ $2 r K D \tilde{r}^{-1}=\eta$, we can estimate

$$
\begin{aligned}
B(x, r) \cap \psi(X) & \subset B(z, 2 r) \cap \psi(X) \subset \psi\left(B\left(\psi^{-1}(z), 2 r K\left\|\psi^{\prime}\right\|^{-1}\right)\right) \\
& \subset \psi\left(B\left(y, 2 \tilde{r} K\left\|\psi^{\prime} \mid\right\|^{-1}+2 \tilde{r} K\left\|\psi^{\prime}\right\|^{-1} D^{2}\right)\right) \\
& \subset \psi\left(B\left(y, 4 D^{2} K\left\|\psi^{\prime}\right\|^{-1} \tilde{r}\right)\right)
\end{aligned}
$$

So, by assumptions of the lemma,

$$
\begin{aligned}
m(B(x, r) \cap \psi(X)) & \leq\left\|\psi^{\prime}\right\|^{h} m\left(B\left(y, 4 D^{2} K\left\|\psi^{\prime}\right\|^{-1} \tilde{r}\right)\right) \\
& \leq\left\|\psi^{\prime}\right\|^{h} L\left(4 D^{2} K\left\|\psi^{\prime}\right\|^{-1} \tilde{r}\right)^{h}=L\left(8 D^{3} K^{2} \eta^{-1}\right)^{h} r^{h}
\end{aligned}
$$

Therefore $m\left(B(x, r) \leq \# R^{*} L\left(8 D^{3} K^{2} \eta^{-1}\right)^{h} r^{h} \leq\left(2 D^{2}\right)^{d} \beta^{-1} L\left(8 D^{3} K^{2} \eta^{-1}\right)^{h} r^{h}\right.$ and applying Theorem 2.8 finishes the proof.

Lemma 4.12. If $S=\left\{\phi_{i}: i \in I\right\}$ is a regular c.i.f.s. and there exist a sequence of points $z_{j} \in J$ and a sequence of positive reals $\left\{r_{j}: j \geq 1\right\}$ such that $B\left(z_{j}, r_{j}\right) \subset X$ and

$$
\liminf _{j \rightarrow \infty} \frac{m\left(B\left(z_{j}, r_{j}\right)\right)}{r_{j}^{h}}=0
$$

then $\Pi_{h}(J)=\infty$.

Proof. For every $\tau \in I^{*}$ denote by $R_{\tau}$ the set $R$ defined in the proof of of Lemma 4.3. It has been shown there that $\mu\left(R_{\tau}\right)=1$, and therefore, as $I^{*}$ is countable, $\mu\left(\bigcap_{\tau \in I^{*}} R_{\tau}\right)=$ 1. Thus, it follows from Lemma 3.7 that $m\left(J_{*}\right)=1$, where $J_{*}=\pi\left(\bigcap_{\tau \in I^{*}} R_{\tau}\right)$ is the set of those points $x \in J$ that can be expressed as $x=\pi(\omega)$ and each element of $I^{*}$ appears in $\omega$ infinitely often. Fix $\varepsilon>0$. By the assumption there exists $z=z_{j}=\pi(\rho)$ and $r=r_{j} / 2$ such that $m(B(z, 2 r)) r^{-h} \leq K^{-h} \varepsilon$. Take $p \geq 1$ so large that $\operatorname{diam}\left(\phi_{\left.\rho\right|_{p}}(X)\right)<r$. By the definition of $J_{*}$ there exists $q \geq 1$ such that $\left.\omega\right|_{[q+1, q+p]}=\left.\rho\right|_{p}$. Thus $\operatorname{dist}\left(\pi\left(\sigma^{q}(\omega)\right), z\right) \leq$ 
$\operatorname{diam}\left(\phi_{\left.\rho\right|_{p}}(X)\right)<r$, whence $B\left(\pi\left(\sigma^{q}(\omega)\right), r\right) \subset X$. Therefore using (BDP.3) first and then (BDP.1), and conformality of $m$

$$
\begin{aligned}
m\left(B\left(\pi(\omega), K^{-1}\left\|\phi_{\left.\omega\right|_{q}}^{\prime}\right\| r\right)\right) & \leq m\left(\phi_{\left.\omega\right|_{q}}\left(B\left(\pi\left(\sigma^{q}(\omega)\right), r\right)\right)\right) \leq\left\|\phi_{\left.\omega\right|_{q}}^{\prime}\right\|^{h} m\left(B\left(\pi\left(\sigma^{q}(\omega)\right), r\right)\right) \\
& \leq\left\|\phi_{\left.\omega\right|_{q}}^{\prime}\right\|^{h} m(B(z, 2 r)) \leq\left\|\phi_{\left.\omega\right|_{q}}^{\prime}\right\|^{h} K^{-h} \varepsilon r^{h}=\left(K^{-1}\left\|\phi_{\left.\omega\right|_{q}}^{\prime}\right\| r\right)^{h} \varepsilon
\end{aligned}
$$

Since $m\left(J_{*}\right)=1$, the application of Theorem 2.9(1) finishes the proof.

Lemma 4.13. Suppose that $S$ is a regular c.i.f.s. and there exists $z \in X(\infty)$ such that $\limsup _{r \rightarrow 0} m(B(z, r)) / r^{h}=0$. Suppose also that there exists an open cone $C(z) \subset X$ with the vertex $z$ such that $z \in \overline{J \cap C(z)}$. Then $\Pi_{h}(J)=\infty$.

Proof. By the second assumption there exists an infinite sequence $z_{j} \in J \cap C(z)$ such that $\lim z_{j}=z$. Since $C(z)$ is a cone there exists $0<\alpha \leq 1$ such that for all $j$ sufficiently large, passing to a subsequence we can suppose that for all $j$, we have $B\left(z_{j}, \alpha\left|z_{j}-z\right|\right) \subset$ $C(z)$. Hence $B\left(z_{j}, \alpha\left|z_{j}-z\right|\right) \subset X$ and $m\left(B\left(z_{j}, \alpha\left|z_{j}-z\right|\right)\right) \leq m\left(B\left(z,(1+\alpha)\left|z_{j}-z\right|\right)\right)$. Therefore, in view of the first assumption of Lemma 4.13 the assumptions of Lemma 4.12 are satisfied and the proof is completed.

As an immediate consequence of this lemma and Corollary 3.24 we get the following.

Corollary 4.14. Suppose that $S$ is a c.i.f.s. and $X$ is a compact nondegenerate subinterval of the real line. If there exists $z \in X(\infty)$ such that $\lim _{\sup _{r \rightarrow 0}} m(B(z, r)) / r^{h}=$ 0 , then $\Pi_{h}(J)=\infty$.

Lemma 4.15. If $S=\left\{\phi_{i}: i \in I\right\}$ is a regular c.i.f.s., there exist a sequence of points $z_{j} \in X(\infty)$ and a sequence of positive reals $\left\{r_{j}: j \geq 1\right\}$ such that $\lim _{j \rightarrow \infty} m\left(B\left(z_{j}, r_{j}\right)\right) / r_{j}^{h}$ $=0$, then $\Pi_{h}(J)=\infty$.

Proof. By the assumption of the lemma there exists $\beta>0$ such that $\phi_{i}(B(z, \beta)) \cap J=$ $\phi_{i}(J \cap B(z, \beta))$ and therefore for every $0<r \leq \beta$ we have $m\left(\phi_{i}(B(z, r))\right)=\int_{B(z, r)}\left|\phi_{i}^{\prime}\right|^{h} d m$. Let $J_{\infty}$ be the subset of those points $x$ in $J$ that can be expressed as $x=\pi(\omega)$, where each element of $I$ appears in $\omega$ infinitely often. So, for every $x \in J_{\infty}$ and every $r>0$ there exists $q \geq 1$ such that $\operatorname{dist}\left(z, \pi\left(\sigma^{q}(\omega)\right)\right)<r$ and $\operatorname{diam}\left(\phi_{\omega_{q+1}}(X)\right)<r$. The rest of the proof is the same as the corresponding part of the proof of Lemma 4.12.

Theorem 4.16. If $S$ is irregular, then either measure $H_{g}(J)$ or $\Pi_{g}(J)$ is either zero or infinity for every gauge function $g$ of the form $t^{h} L(t)$, where $L(t)$ is a slowly varying function. Additionally $H_{h}(J)=0$.

Proof. Suppose that a measure $\mathrm{H}_{g}(J)$ or $\Pi_{g}(J)$ (call it $G_{g}$ ) is finite. Then the Jacobian (Radon-Nikodym derivative) of a map $\phi_{\omega}, \omega \in I^{*}$, with respect to the measure $G_{g}$ is equal to $\left|\phi_{\omega}^{\prime}\right|^{h}$. By the definition of pressure there exists $n_{0} \geq 1$ such that $\sum_{\omega \in I^{n}}\left\|\phi_{\omega}^{\prime}\right\|^{h}<\exp (n \mathrm{P}(h) / 2)$ for every $n \geq n_{0}$. Hence

$$
G_{g}(J) \leq \sum_{\omega \in I^{n}} G_{g}\left(\phi_{i}(J)\right) \leq \sum_{\omega \in I^{n}}\left\|\phi_{\omega}^{\prime}\right\|^{h} m(J)<\exp (n \mathrm{P}(h) / 2) G_{g}(J)
$$


Thus letting $n \rightarrow \infty$ and noting that by Theorem $3.21 \mathrm{P}(h)<0$, we obtain $G_{g}(J)=0$. The proof that $H_{h}(J)=0$ is very similar but requires slightly different argument as we do not know whether $\mathrm{H}_{h}(J)$ is finite. Indeed, if $n \geq n_{0}$ is as above, then

$$
\sum_{\omega \in I^{n}}\left(\operatorname{diam}\left(\phi_{i}(X)\right)\right)^{h} \leq \sum_{\omega \in I^{n}} D^{h}\left\|\phi_{\omega}^{\prime}\right\|^{h}<D^{h} \exp (n \mathrm{P}(h) / 2)
$$

and letting $n \rightarrow \infty$ we conclude that $\mathrm{H}_{h}(J)=0$.

Theorem 4.17. If $S$ is a c.i.f.s., $g(t)$ is a gauge function of the form $t^{h} L(t)$, where $L(t)$ is a slowly varying function, and one of the numbers $H_{g}(J)$ or $\Pi_{g}(J)$ is positive and finite, then the system $S$ is regular and the conformal measure $m$ is up to a multiplicative constant either equal to $H_{g}$ or $\Pi_{g}$.

Proof. That the system $S$ is regular follows immediately from Theorem 4.16. The other part of the theorem follows now from Lemma 3.10 applied with the measure $\nu$ being either $\mathrm{H}_{g} / H_{g}(J)$ or $\Pi_{g} / \mid P i_{g}(J)$ and from Theorem 3.18.

As an immediate consequence of this theorem and Lemma 3.14 we get the following.

Corollary 4.18. If a c.i.f.s. $S$ is finite, then, up to a multiplicative constant, the following three measures on $J$ are equal: $H_{h}, \Pi_{h}$, and the conformal measure $m$.

\section{Examples}

In this section we provide a number of examples of infinite c.i.f.s. showing how flexible, how large a variety of fractal features one can meet among them.

Example 5.1. ( $J$ is an $F_{\sigma \delta}$ but not a $G_{\delta}$ ). Denote by $Q$ the set of all rational numbers in $[0,1]$. Let $X=[0,1] \times[0,1]$ and let $\Delta=\{(x, x) \in X\}$ be the diagonal of $X$. Consider a c.i.f.s. $\left\{\phi_{i}: X \rightarrow X: i \in Q \cup\{-1\}\right\}$ consisting of linear mappings and such that

(a) $\phi_{i}(X) \cap \Delta=\left\{\phi_{i}(0,1)\right\}=\{(i, i)\}$ for all $i \in Q$

(b) $\phi_{-1}(x, y)=(x / 2,(y+1) / 2)$

(c) The sets $\phi_{i}(X), i \in Q \cup\{-1\}$, are mutually disjoint.

Then $J \cap \Delta=Q$ is not $G_{d}$, so neither is $J$. Let us also note that this system is not locally finite.

Example 5.2. $(\mathrm{PD}(J) \geq \underline{\mathrm{BD}}(J)>\mathrm{HD}(J))$. Take any sequence of positive numbers $\left\{r_{i}: i \geq 1\right\}$ (for example of the form $b^{i}, 0<b<1$ ) such that the equation $\sum_{i>1} r_{i}^{t}=1$ has a (unique) solution and this solution is less than 1. Consider a family $\left\{\phi_{i}:\{z \in \mathbb{C}:\right.$ $|z| \leq 1\} \rightarrow\{z \in \mathbb{C}:|z| \leq 1\}: i \geq 1\}$ of similarity maps satisfying the (OSC) and such that $\left\|\phi_{i}^{\prime}\right\|=r_{i}$ and $X(\infty)=\{z:|z|=1\}$. Then by Theorem $2.8, \operatorname{PD}(J) \geq \underline{\operatorname{BD}}(J) \geq$ $\underline{\mathrm{BD}}(X(\infty)) \geq 1$ and by Theorem $3.15, \operatorname{HD}(J)<1$. 
Example 5.3. (Irregular system). Such a system has been described in Ex. 4.5 of [17]. Since this is a very short and important example we repeat here its construction. Let $I=\left\{(n, k): n \geq 1\right.$ and $\left.1 \leq k \leq 2^{n^{2}-1}\right\}$, let $X=[0,1]$, and let $S=\left\{\phi_{n, k}: X \rightarrow X:\right.$ $(n, k) \in I\}$ be a system consisting of similarity maps $\phi_{n, k}$ such that $\left\|\phi_{n, k}^{\prime}\right\|=2^{-\left(n^{2}+n\right)}$ and such that the intervals $\phi_{n, k}(X)$ are mutually disjoint. This last requirement can be satisfied since $\sum_{(n, k) \in I}\left\|\phi_{n, k}^{\prime}\right\|=\sum_{n \geq 1} 2^{-\left(n^{2}+n\right)} 2^{n^{2}-1}=1 / 2<1$. Notice that by this computation we have shown that $\psi(1)=1 / 2<1$. Observe also that $\psi(t)=\sum_{n \geq 1} 2^{n^{2}-1} 2^{-\left(n^{2}+n\right) t}=$ $\sum_{n \geq 1} 2^{n^{2}(1-t)-n t-1}=\infty$ for all $0<t<1$. Thus, in view of Theorem 3.21 $S$ is irregular, in view of Theorem $3.22 h=\operatorname{HD}(J)=1$, and in view of Theorem 4.16, $J$ is dimensionless in the restricted sense.

Example 5.4. (Linear, regular but not hereditarily regular). This example is very similar to the Example 5.3. The only difference in its definition is that now we take $I=\left\{(n, k): n \geq 1\right.$ and $\left.1 \leq k \leq 2^{n^{2}}\right\}$. Then the same computations as in Example 5.3 above show that $\psi(1)=1$, thus $\mathrm{P}(1)=0$, and $\psi(t)=\infty$ for all $0<t<1$. Hence, in view of Theorem 4.5, $S$ is regular, the only conformal measure is the Lebesgue measure $\lambda_{1}$, and $h=\mathrm{HD}(J)=1$. Moreover in view of Theorem $3.20, S$ is not hereditarily regular.

Notice that Example 5.4 provides a number of irregular examples. In fact every cofinite subsystem of $S$ is irregular.

Example 5.5. (Hereditarily regular linear system with $0<\Pi_{h}(J)<\infty, \mathrm{H}_{h}(J)=0$ ). Let $X=[0,1]$ and let $S=\left\{\phi_{n}: X \rightarrow X: n \geq 1\right\}$ be the c.i.f.s. consisting of similarities $\phi_{n}(x)=\frac{x}{3 n^{2}}+\frac{1}{n}-\frac{1}{3 n^{2}}$ so that $\phi_{n}(0)=\frac{1}{n}-\frac{1}{3 n^{2}}$ and $\phi_{n}(1)=\frac{1}{n}$. Thus $\left\|\phi_{n}^{\prime}\right\|=\frac{1}{3 n^{2}}$ and $\psi(t)=\sum_{n \geq 1}\left\|\phi_{n}^{\prime}\right\|^{t}=\sum_{n \geq 1} 3^{-t} n^{-2 t}$. Hence $h=\operatorname{HD}(J)>1 / 2$ and by Theorem $3.20 S$ is hereditarily regular. Let $m$ be the corresponding conformal measure. Then for every $n \geq 1$

$$
m(B(0,1 / n))=\sum_{k \geq n}\left(\frac{1}{3 k^{2}}\right)^{h} \geq 3^{-h} \int_{n}^{\infty} x^{-2 h} d x=3^{-h} \frac{1}{2 h-1}\left(\frac{1}{n}\right)^{2 h-1} .
$$

Taking now for any $0<r \leq 1$ the unique integer $n \geq 1$ such that $1 /(n+1)<r \leq 1 / n$, we get $m(B(0, r)) \geq C r^{2 h-1}$, where $C=\left((2 h-1) 3^{h} 2^{2 h-1}\right)^{-1}$. Since $h-1<0$ it now follows from Lemma 4.9 that $H_{h}(J)=0$. Positivity of $\Pi_{h}(J)$ is guaranteed by Lemma 4.3. We now show that the assumptions of Lemma 4.10 are satisfied with $\gamma=3$ if for every $n \geq 1$ the point $y$ is chosen to be $1 / n$. Indeed, fix $n \geq 1$ and take $1 / n^{2} \leq r \leq 1$. Suppose first that $r \leq 1 /(2 n)$. Then $n \geq 2$ and $\frac{1}{n}-r>\frac{1}{2 n}$. Let $I(r)=\left\{k \geq 1: \frac{1}{k} \leq \frac{1}{n}\right.$ and $\left.\frac{1}{k+1} \geq \frac{1}{n}-r\right\}$. Notice that $\# I(r) \geq(1 / n-r)^{-1}-n=n^{2} r /(1-n r) \geq n^{2} r$. Therefore

$$
\begin{aligned}
m(B(1 / n, r)) & \geq \sum_{k \in I(r)}\left(\frac{1}{3 k^{2}}\right)^{h} \geq\left(\frac{1}{3(2 n)^{2}}\right)^{h} \# I(r) \geq(12)^{-h} n^{-2 h} n^{2} r \geq \\
& =(12)^{-h}\left(\frac{1}{n^{2}}\right)^{h-1} r \geq(12)^{-h} r^{h-1} r=(12)^{-h} r^{h} .
\end{aligned}
$$


Now suppose that $1 /(2 n) \leq r \leq 2 / n$. Then $1 / n^{2} \leq r / 4 \leq 1 /(2 n)$ and in view of the previous case $m(B(1 / n, r)) \geq m(B(1 / n, r / 4)) \geq(12)^{-h}(r / 4)^{h}=(48)^{-h} r^{h}$. Finally suppose that $r \geq 2 / n$. Then $B(1 / n, r) \supset B(0, r / 2) \geq C(r / 2)^{2 h-1}=2 C 4^{-h} r^{h} r^{h-1}$. Thus the assumptions of Lemma 4.10 are satisfied and therefore $\Pi_{h}(J)<\infty$.

We should mention here that in the next section the c.i.f.s. induced by complex continued fractions will be considered which is also hereditarily regular and whose limit set has $h$-dimensional Hausdorff measure 0 and of $h$-dimensional finite packing measure. The idea for proving these properties will be the same there as in Example 5.5.

Example 5.6. (Hereditarily regular linear system with $\Pi_{h}(J)=\infty, \mathrm{H}_{h}(J)>0$ ). Let $X=[0,1]$ and let $S=\left\{\phi_{n}: X \rightarrow X: n \geq 1\right\}$ be the c.i.f.s. consisting of similarities $\phi_{n}(x)=2^{-2 n} x+2^{-n}-2^{-2 n}$ so that $\phi_{n}(0)=2^{-n}-2^{-2 n}$ and $\phi_{n}(1)=2^{-n}$. Thus $\left\|\phi_{n}^{\prime}\right\|=2^{-2 n}$ and $\psi(t)=\sum_{n \geq 1}\left\|\phi_{n}^{\prime}\right\|^{t}=\sum_{n \geq 1} 2^{-2 n t}$. Hence $h=1 / 2$ and by Theorem 3.20 $S$ is hereditarily regular. Let $m$ be the corresponding conformal measure. Then for every $n \geq 1$ we have $m\left(B\left(0,2^{-n}\right)\right)=\sum_{k \geq n} 2^{-2 k h}=2\left(2^{-2 n h}\right)$. Taking now for any $0<r \leq 1 / 2$ the unique integer $n \geq 1$ such that $2^{-(n+1)}<r \leq 2^{-n}$, we get

$$
m(B(0, r)) \leq 4 r^{2 h}
$$

Thus, $\Pi_{h}(J)=\infty$ by Corollary 4.14. Finiteness of $\mathrm{H}_{h}(J)$ is guaranteed by Lemma 4.2. We now show that the assumptions of Lemma 4.11 are satisfied with $\gamma=1$ if for every $n \geq 1$ the point $y$ is chosen to be $2^{-n}$. Indeed, fix $n \geq 1$ and take $2^{-2 n} \leq r \leq 1 / 2$. If $r \geq 2^{-n}$, then $m\left(B\left(2^{-n}, r\right)\right) \leq m(B(0,2 r)) \leq 4(2 r)^{2 h}=8 r^{2 h}$. In general case $r^{1 / 2} \geq 2^{-n}$ and then $m\left(B\left(2^{-n}, r\right)\right) \leq m\left(B\left(2^{-n}, r^{1 / 2}\right)\right) \leq 8\left(r^{1 / 2}\right)^{2 h}=8 r^{h}$. Thus the assumptions of Lemma 4.11 are satisfied and therefore $\mathrm{H}_{h}(J)>0$.

Example 5.7. (Hereditarily regular linear system with $\left.\Pi_{h}(J)=\infty, \mathrm{H}_{h}(J)=0\right)$. This example is made up by gluing together Examples 5.5 and 5.6. Namely let $X=[0,2]$ and $S=\left\{\phi_{n, 0}, \phi_{n, 1}: n \geq 2\right\}$, where $\phi_{n, 0}(x)=\frac{1}{3 n^{2}} \frac{x}{2}+\frac{1}{n}-\frac{1}{3 n^{2}}$ and $\phi_{n, 1}(x)=2^{-2 n} \frac{x}{2}+$ $2^{-n}-2^{-2 n}+1$. Then $\phi_{n, 0}([0,2])=\left[\frac{1}{n}-\frac{1}{3 n^{2}}, \frac{1}{n}\right] \subset[0,1 / 2]$ and $\phi_{n, 1}([0,2])=\left[2^{-n}-2^{-2 n}+\right.$ $\left.1,2^{-n}+1\right] \subset[1,2]$ and $\psi(t)=\sum_{n>2}\left(\left\|\phi_{n, 0}^{\prime}\right\|^{t}+\left\|\phi_{n, 1}^{\prime}\right\|^{t}\right)=\sum_{n>2} 6^{-t} n^{-2 t}+2^{-t} 2^{-2 n t}$. So, the interval of convergence of $\psi(t)$ is $(1 / 2, \infty)$. Thus, in view of Theorem 3.20, $S$ is hereditarily regular and $h=\operatorname{HD}(J)>1 / 2$. We see that $X(\infty)=\{0,1\}$ and if $m$ is the corresponding $h$-conformal measure, then as in Example 5.5 we get $m(B(0,1 / n)) \geq$ $(2 h-1)^{-1}(1 / n)^{2 h-1}$ which in view of Lemma 4.8 implies that $\mathrm{H}_{h}(J)=0$ and as in Example 5.6 we get $m\left(B\left(1,2^{-n}\right)\right) \leq 4^{h}\left(4^{h}-1\right)^{-1}\left(2^{-n}\right)^{2 h}$ which in view of Corollary 4.14 implies that $\Pi_{h}(J)=\infty$.

Example 5.8. (One-dimensional systems). Here we want to describe how every compact subset $F$ of the interval $X=[0,1]$ gives canonically rise to a linear c.i.f.s. on $X$ such that $X(\infty)=(\partial F)^{d}=\{x \in X: x$ is an accumulation point of $\partial F\}$, the CantorBendixon derived set of $\partial F$. Indeed, let $R$ be the family of all connected components of $X \backslash F$ and for every $C \in R$ let $\phi_{C}: X \rightarrow X$ be the unique linear map such that $\phi_{C}(0)$ is 
the left endpoint of the closure of $C$ and $\phi_{C}(1)$ is the right endpoint of the closure of $C$. The system $S=\left\{\phi_{C}: X \rightarrow X: C \in R\right\}$ has the property required.

Example 5.9. We want to describe here an example of a hereditarily regular c.i.f.s. $S$ such that

(a) Condition (c) of Lemma 2.2 is not satisfied.

(b) Condition (a) of Lemma 2.2 is satisfied for every finite subsystem of $S$.

(c) The Perron-Frobenius operator is almost periodic.

Indeed, let $\left\{r_{n}: n \geq 1\right\}, 0<r_{n}<1$, be any sequence of real numbers such that $\sum_{n>1} r_{n}<1$ and the set of parameters $t$ for which the series $\sum_{n>1} r_{n}^{t}$ converges, is open. Let $\left\{\left[a_{n} . b_{n}\right]: n \geq 1\right\}$ be a countable disjoint family of closed subintervals of the interval $[0,1]$ such that $b_{n}-a_{n}=r_{n}$ for all $n \geq 1$. Finally let $\left\{g_{n}:[-1,2] \rightarrow[1,2]: n \geq 1\right\}$ be a familly of continuous functions that are constant on each interval of the form $\left[a_{k}, b_{k}\right]$, $k \geq 1$. Moreover we can choose these functions $g_{n}$ not to form an equicontinuous family, and with a suitable choice of numbers $r_{n}$ and suitable placing of intervals $\left[a_{n}, b_{n}\right]$ we can require all $g_{n}$ to be Lipshitz continuous.

Now we have all ingredients needed to define our system $S$. We declare $X=[0,1]$, $V=(-1,2)$, and for every $n \geq 1$ we define the map $\phi_{n}:[-1,2] \rightarrow \mathbb{R}$, setting

$$
\phi_{n}(x)=a_{n}+m_{n} \int_{-1}^{x} g_{n}(t) d t
$$

where $m_{n}=\left(b_{n}-a_{n}\right)\left(\int_{-1}^{2} g_{n}(t) d t\right)^{-1}$. Note that $\phi_{n}(-1)=a_{n}$ and $\phi_{n}([-1,2])=\left[a_{n}, b_{n}\right]$. Since $\phi_{n}^{\prime}(x)=m_{n} g_{n}(x) \leq \frac{2}{3}\left(b_{n}-a_{n}\right)<2 / 3$, the maps $\phi_{n}, n \geq 1$, form an iterated function system and in order to show its conformality our only task is to check that (BDP) holds. Indeed, for all integers $n, k \geq 1$ set $\gamma(n, k)=m_{n} g_{n}\left(a_{k}\right)$. Then for every $n \geq 1$, every $\omega \in I^{n}$, and every $x \in[0,1]$ we have

$$
\phi_{\omega}^{\prime}(x)=\gamma\left(\omega_{1}, \omega_{2}\right) \gamma\left(\omega_{2}, \omega_{3}\right) \ldots \gamma\left(\omega_{n-2}, \omega_{n-1}\right) \gamma\left(\omega_{n-1}, \omega_{n}\right) m_{\omega_{n}} g_{\omega_{n}}(x)
$$

But since $g_{k}(y) / g_{k}(x) \leq 2$ for all $k \geq 1$ and all $x, y \in[-1,2]$, we see that $\left|\phi_{\omega}^{\prime}(y)\right| /\left|\phi_{\omega}^{\prime}(x)\right|=$ $g_{\omega_{n}}(y) / g_{\omega_{n}}(x) \leq 2$ for every $n \geq 1$, and every $\omega \in I^{n}$. The proof of (BDP) is finished.

The almost periodicity of the Perron-Frobenius operator also follows from (5.1) since then all its iterates are constant on the segments of the form $\left[a_{n}, b_{n}\right]$ and therefore we can apply Lemma a.6.

In order to see that the system $S$ is hereditarily regular note that $r_{n} / 6=\left(b_{n}-a_{n}\right) / 6 \leq$ $\left\|\phi_{n}^{\prime}\right\| \leq \frac{2}{3}\left(b_{n}-a_{n}\right)=\frac{2}{3} r_{n}$. Therefore $F(S)$ is an open set and the required property follows from Theorem 3.20.

Now, that condition (a) of Lemma 2.2 is satisfied for every finite subsystem of $S$ follows immediately from the properties of $g_{n}$ and the formula $\phi_{n}^{\prime}(x)=m_{n} g_{n}(x)$. Since $\left.\log \left|\phi_{n}^{\prime}(y)\right|-\log \left|\phi_{n}^{\prime}(x)\right|=\log \left(g_{n}(y)\right)-\log \left(g_{n}(x)\right)=\beta\left(g_{n}(y)-g_{(} x\right)\right)$ with some $1 / 2 \leq \beta \leq 1$, and since the family $\left\{g_{n}:[-1,2] \rightarrow[1,2]: n \geq 1\right\}$ is not equicontinuous, we see that property (c) of Lemma 2.2 fails. 


\section{Complex continued fractions}

In this section we focus our attention on a special infinite iterated function system, introduced and studied in [11], that is generated by complex continued fractions. Namely,

let $I=\{m+n i:(m, n) \in \mathbb{I N} \times \boldsymbol{Z}\}$, where $\boldsymbol{Z}$ is the set of integers and $\mathbb{I N}$ is the set of positive integers. Let $X \subset \mathbb{C}$ be the closed disc centered at the point $1 / 2$ with radius $1 / 2$ and let $V=B(1 / 2,3 / 4)$. For $b \in I$ we define $\phi_{b}: V \rightarrow V$ putting

$$
\phi_{b}(z)=\frac{1}{b+z}
$$

The following figures illustrate the construction of $J$.

\section{FIGURES GO HERE}


Using the Koebe distortion theorem the following properties can be easily checked by a straightforward direct computation. There exists a constant $K \geq 1$ such that for every $b \in I$

$$
\begin{gathered}
\phi_{b}(V) \subset B\left(0, K|b|^{-1}\right) \\
K^{-1}|b|^{-2} \leq\left|\phi_{b}^{\prime}(z)\right| \leq K|b|^{-2} \\
K^{-1}|b|^{-2} \leq \operatorname{diam}\left(\phi_{b}(V)\right) \leq K|b|^{-2}
\end{gathered}
$$

One would like to call the collection of mappings $\left\{\phi_{b}: b \in I\right\}$ a conformal iterated function system. This however is not quite possible as $\phi_{1}^{\prime}(0)=-1$ and condition (2.1) fails. Nevertheless, since it is satisfied for the system $\left\{\phi_{b} \circ \phi_{c}:(b, c) \in I \times I\right\}$, in the sequel we will treat the family $\left\{\phi_{b}: b \in I\right\}$ as a c.i.f.s.. Let $J$ denote the associated limit set. We begin with the following.

Proposition 6.1. The system $S=\left\{\phi_{b}: b \in I\right\}$ is hereditarily regular and $\theta(S)=1$.

Proof. For every $n \geq 0$ set $I(n)=\left\{b \in I: 2^{n} \leq|b|<2^{n+1}\right\}$. Notice that there exists $\alpha \geq 1$ such that $\alpha^{-1} 4^{n} \leq \# I(n) \leq 4 \alpha \cdot 4^{n}$. Therefore $\left(4^{t} \alpha\right)^{-1} \sum_{n \geq 0} 4^{(1-t) n} \leq$ $4^{-t} \sum_{n \geq 0} \# I(n) 4^{-t n} \leq \sum_{b \in I}|b|^{-2 t} \leq \sum_{n \geq 0} \# I(n) 4^{-t n} \leq 4 \alpha \sum_{n \geq 0} 4^{(1-t) n}$ for all $t \geq 0$. Hence, using (6.2) we get $\left(4^{t} K^{t} \alpha\right)^{-1} \sum_{n>0} 4^{(1-t) n} \leq \psi(t) \leq 4 \alpha \bar{K}^{t} \sum_{n>0} 4^{(1-t) n}$. Thus $\theta(S)=1, \psi(\theta)=\infty$, and in view of Theorem 3.20 our system is hereditarily regular.

In view of this proposition, the last assertion of Theorem 3.20, and Theorem 4.5 we get the following result which was first proved in [11] with different methods.

Theorem 6.2. $1<h=H D(J)<2$. 
Let $m$ be the $h$-conformal measure associated to the system $S=\left\{\phi_{b}: b \in I\right\}$. The proof of the following lemma is based on the same idea as the proof of Proposition 6.1.

Lemma 6.3. There exists a constant $Q>0$ such that for every $0<r \leq 1$ we have $m\left(B(0, r) \geq Q r^{2 h-2}\right.$.

Proof. Let $I(r)=\left\{b \in I: r / 2<K|b|^{-1}<r\right\}=\left\{b \in I: K r^{-1}<|b|<2 K r^{-1}\right\}$. Observe that there exists $\alpha>0$ so small that $\# I(r) \geq \alpha\left(K r^{-1}\right)^{2}$. Therefore using (6.1)(6.3) we get

$$
m(B(0, r)) \geq \# I(r) K^{-h}(2 K)^{-2 h} r^{2 h} \geq \alpha 2^{-2 h} K^{2-3 h} r^{2 h-2}
$$

The proof is finished.

Since $0 \in X(\infty)$ and since $h<2$, this lemma and Lemma 4.9 give the following.

Theorem 6.4. $H_{h}(J)=0$.

Now, we shall prove the following.

Theorem 6.5. $0<\Pi_{h}(J)<+\infty$.

Proof. Positiveness of $\Pi_{h}(J)$ follows from Lemma 4.3. In order to prove that $\Pi_{h}(J)$ is finite we will check that the assumptions of Lemma 4.10 are satisfied. So, let $b \in I$ and let $x \in \phi_{b}(V)$. Consider $|x|^{2} \leq r \leq 1 / 64$. Define $I(x, r)=\left\{a \in I: \phi_{a}(X) \subset B(x, r)\right\}$. Let $\operatorname{In}(z)$ be the inverse function, $\operatorname{In}(z)=1 / z$. Notice that if $0<r<|x|$, then

$$
\operatorname{In}(B(x, r))=B\left(\frac{|x|^{2}}{|x|^{2}-r^{2}} \frac{1}{x}, \frac{r}{|x|^{2}-r^{2}}\right)
$$

and

$$
I(x, r)=\{a \in I: \operatorname{In}(B(x, r)) \supset B(a+1 / 2,1 / 2)\}
$$

Suppose first that $r \leq|x| / 2$. Then $|x|^{2}-r^{2} \geq 3|x|^{2} / 4$ and therefore

$$
\frac{|x|}{|x|^{2}-r^{2}} \leq \frac{4}{3} \frac{1}{|x|}
$$

Let now $I_{1}=\left\{a \in I(x, r):|a|<\frac{|x|}{|x|^{2}-r^{2}}\right\}$. Since $|x| /\left(|x|^{2}-r^{2}\right)$ is the modulus of the center of the ball $\operatorname{In}(B(x, r))$, there exists $\beta>0$ such that $\# I_{1} \geq \beta r^{2} /\left(|x|^{2}-r^{2}\right) \geq \beta r^{2} /|x|^{2}$. Therefore by (6.2), (6.5) and since $r \geq|x|^{2}$ we may write

$$
\begin{aligned}
m(B(x, r)) & \geq \# I_{1} K^{-h}(9 / 16)^{h}|x|^{2 h} \geq \beta(2 K)^{-h}|x|^{2 h} \frac{r^{2}}{|x|^{2}} \\
& =\beta(2 K)^{-h}|x|^{2 h-4} r^{2} \geq \beta(2 K)^{-h} r^{h-2} r^{2} \\
& =\beta(2 K)^{-h} r^{h}
\end{aligned}
$$


Now suppose that $|x| / 2 \leq r \leq 2|x|$. Then $|x|^{2} \leq r / 4 \leq|x| / 2$ and therefore by $(6.6)$

$$
m(B(x, r)) \geq m(B(x, r / 4)) \geq \beta(8 K)^{-h} r^{h}
$$

Finally if $r \geq 2|x|$, then $B(x, r) \supset B(0, r / 2)$. Thus, in view of Lemma 6.3 , and since $h<2$, we can estimate

$$
m(B(x, r)) \geq Q(r / 2)^{2 h-2}=Q 4^{1-h} r^{h-2} r^{h} \geq Q 4^{1-h} r^{h}
$$

Combining this estimate, (6.7), and (6.6) we conclude that the assumptions of Lemma 4.10 are satisfied which completes the proof.

The rest of this section is devoted to estimating the Hausdorff dimension $h$ of the limit set $J$ of complex continued fractions improving quantitatively Theorem 6.2. Let us state our estimates as a theorem.

Theorem 6.6. If $J$ is the limit set of complex continued fractions, then

$$
1.2484<h=H D(J)<1.9
$$

Proof. Note that, if $\omega=\left(b_{1}, b_{2}, \ldots, b_{n}\right) \in I^{n}$, then

$$
\phi_{\omega}(z)=\frac{p_{n-1}(\omega) z+p_{n}(\omega)}{q_{n-1}(\omega) z+q_{n}(\omega)}
$$

where $p_{0}(\omega)=0, p_{1}(\omega)=q_{0}(\omega)=1$, and $q_{1}(\omega)=b_{1} ; q_{k}(\omega)=b_{k} q_{k-1}(\omega)+q_{k-2}(\omega)$, $p_{k}(\omega)=b_{k} p_{k-1}(\omega)+p_{k-2}(\omega), 2 \leq k \leq n$. Frequently we will write $q_{n}$ and $p_{n}$ only instead of $q_{n}(\omega)$ and $p_{n}(\omega)$. With this convention $\phi_{\omega}(X)$ is the ball with center

$$
\frac{2 q_{n-1}\left|q_{n-1}+4 q_{n}\right|^{2}\left(p_{n} q_{n-1}-p_{n-1} q_{n}\right)+p_{n-1}\left(\left|q_{n-1}+4 q_{n}\right|^{2}-4\left|q_{n-1}\right|^{2}\right)\left(q_{n-1}^{2}+2 q_{n-1} q_{n}\right)}{q_{n-1}\left(\left|q_{n-1}+4 q_{n}\right|^{2}-4\left|q_{n-1}\right|^{2}\right)\left(q_{n-1}^{2}+2 q_{n-1} q_{n}\right)}
$$

and with the radius

$$
\frac{2}{\left|q_{n-1}+2 q_{n}\right|^{2}-\left|q_{n-1}\right|^{2}}
$$

Moreover

$$
\phi_{\omega}^{\prime}(z)=\frac{q_{n} p_{n-1}-p_{n} q_{n-1}}{\left(q_{n-1} z+q_{n}\right)^{2}}=\frac{(-1)^{n}}{\left(q_{n-1} z+q_{n}\right)^{2}} \quad \text { and } \quad\left|\phi_{\omega}^{\prime}(z)\right|=\frac{1}{\left|q_{n-1}\right|^{2}\left|z+\frac{q_{n}}{q_{n-1}}\right|^{2}}
$$

Set $w=q_{n} / q_{n-1}$ and consider the circle with center $w+1 / 2$ and of radius $1 / 2$. Let $\alpha(w+1 / 2)$ and $\beta(w+1 / 2)$ be the two points of this circle lying on the line through 0 and $w+1 / 2$ with $\alpha(w+1 / 2)$ closer to the origin. Then

$$
\left\|\phi_{\omega}^{\prime}\right\|=\frac{1}{\left|q_{n-1}\right|^{2}} \sup \left\{\frac{1}{|z+w|^{2}}:|z-1 / 2| \leq 1 / 2\right\}=\frac{1}{\left|q_{n-1}\right|^{2}} \frac{1}{\alpha^{2}|w+1 / 2|^{2}}
$$


and

$$
\inf \left|\phi_{\omega}^{\prime}\right|=\frac{1}{\left|q_{n-1}\right|^{2}} \inf \left\{\frac{1}{|z+w|^{2}}:|z-1 / 2| \leq 1 / 2\right\}=\frac{1}{\left|q_{n-1}\right|^{2}} \frac{1}{\beta^{2}|w+1 / 2|^{2}}
$$

Since $|\alpha(w+1 / 2)-(w+1 / 2)|=|\beta(w+1 / 2)-(w+1 / 2)|=1 / 2$, this gives

$$
\left\|\phi_{\omega}^{\prime}\right\|=\frac{1}{\left|q_{n-1}\right|^{2}\left(\left|\frac{q_{n}}{q_{n-1}}+\frac{1}{2}\right|-\frac{1}{2}\right)^{2}}=\frac{4}{\left|q_{n}\right|^{2}\left(\left|2+\frac{q_{n-1}}{q_{n}}\right|-\left|\frac{q_{n-1}}{q_{n}}\right|\right)^{2}}
$$

and

$$
\left\|\phi_{\omega}^{\prime}\right\|=\frac{1}{\left|q_{n-1}\right|^{2}\left(\left|\frac{q_{n}}{q_{n-1}}+\frac{1}{2}\right|+\frac{1}{2}\right)^{2}}=\frac{4}{\left|q_{n}\right|^{2}\left(\left|2+\frac{q_{n-1}}{q_{n}}\right|+\left|\frac{q_{n-1}}{q_{n}}\right|\right)^{2}} .
$$

Since $\left|2+q_{n-1} / q_{n}\right|-\left|q_{n-1} / q_{n}\right| \geq 1$ and since $\left|2+q_{n-1} / q_{n}\right|+\left|q_{n-1} / q_{n}\right| \leq 3$, we thus get $\left\|\phi_{\omega}^{\prime}\right\| \leq 4 /\left|q_{n}\right|^{2}$ and $\inf \left|\phi_{\omega}^{\prime}\right| \geq 4 /\left(9\left|q_{n}\right|^{2}\right)$. Set

$$
F_{n}(t)=\sum_{|\omega|=n} \frac{1}{\left|q_{n}(\omega)\right|^{2 t}}
$$

Since $\psi_{n}(t) \leq 4^{t} F_{n}(t)$ and since $\underline{\psi}_{n}(t) \geq(4 / 9)^{t} F_{n}(t)$, it follows from Lemma 4.1(c) that $h$ is the only number $t$ such that for all $n$

$$
4^{-t} \leq F_{n}(t) \leq(9 / 4)^{t}
$$

Thus, if $F_{n}(t)>81 / 16$, then $t<h$ and if $F_{n}(t)<1 / 16$, then $h<t$.

Now let $\gamma_{0}=0<\gamma_{1}<\gamma_{2}<\ldots$ be an increasing sequence of integers. Then summing over sectors we can write

$$
\begin{aligned}
F_{1}(t) & =\sum_{m=1}^{\infty} \frac{1}{m^{2 t}}+\sum_{m=1}^{\infty} \sum_{j=1}^{\infty} \sum_{\gamma_{j-1}} \frac{1}{m<|n| \leq \gamma_{j} m} \frac{1}{\left(m^{2}+n^{2}\right)^{t}} \\
& =\zeta(2 t)+2 \sum_{m=1}^{\infty} \frac{1}{m^{2 t}} \sum_{j=1}^{\infty} \sum_{\gamma_{j-1}} \frac{1}{1+(n / m)^{2 t}}
\end{aligned}
$$

If $m \gamma_{j-1}<|n| \leq m \gamma_{j}$, then $1+\gamma_{j-1}^{2}<1+(n / m)^{2} \leq 1+\gamma_{j}^{2}$. So,

$$
\zeta(2 t)+2 \zeta(2 t-1) \sum_{j=1}^{\infty} \frac{\gamma_{j}-\gamma_{j-1}}{\left(1+\gamma_{j}^{2}\right)^{t}} \leq F_{1}(t) \leq \zeta(2 t)+2 \zeta(2 t-1) \sum_{j=1}^{\infty} \frac{\gamma_{j}-\gamma_{j-1}}{\left(1+\gamma_{j-1}^{2}\right)^{t}}
$$

Choosing the $\gamma_{j}^{\prime}$ s so that $\sum_{j=1}^{\infty}\left(\gamma_{j}-\gamma_{j-1}\right) /\left(1+\gamma_{j}^{2}\right)^{t}$ is as close to 1 as we like we see that $F_{1}(t) \leq \zeta(2 t)+2 \zeta(2 t-1)$. Using $\gamma_{j}=j, j=0,1, \ldots$, we find $\zeta(2 t)+2 \zeta(2 t-$ 1) $\sum_{j=1}^{\infty} \frac{1}{\left(1+j^{2}\right)^{t}} \leq F_{1}(t)$ or $\zeta(2 t)+2 \zeta(2 t-1)(\zeta(2 t)-1) \leq F_{1}(t)$. We find $F_{1}(t) \geq \zeta(2 t)+$ 
$2 \zeta(2 t-1) \sum_{j=1}^{k} \frac{1}{\left(1+j^{2}\right)^{t}}>81 / 16$ with $t=1.2484$ by setting $k=1500$ and evaluating the middle term with Mathematica.

In order to obtain an upper bound on the dimension of $J$ we employ a general geometric technique as in [11] and the properties of pressure function. Namely, if $\sigma$ and $t$ are such numbers that for each $\omega=\left(b_{1}, b_{2}, \ldots, b_{n}\right)$

$$
R(\omega, t)=\sum_{b}\left(\frac{\operatorname{diam}\left(\phi_{\omega b}(D)\right)}{\operatorname{diam}\left(\phi_{\omega}(D)\right)}\right)^{t}<\sigma \leq 1
$$

then $\mathrm{HD}(J) \leq t$. In fact this inequality implies that $\mathrm{P}(t) \leq \log \sigma \leq 0$. In our case $\left.\phi_{\omega}(X)\right)$ is a disc with radius $2 /\left(\left|q_{n-1}+2 q_{n}\right|^{2}-\left|q_{n-1}\right|^{2}\right)$. So,

$$
R(\omega, t)=\sum_{b}\left(\frac{\left|2+q_{n-1} / q_{n}\right|^{2}-\left|q_{n-1} / q_{n}\right|^{2}}{\left|2 b+1+2 q_{n-1} / q_{n}\right|^{2}-1}\right)^{t}
$$

Set

$$
M(k, t)=\max _{z \in D} \sum_{b}\left(\frac{|2+z|^{2}-|z|^{2}}{|2 b+1+2 z|^{2}-1}\right)^{t}
$$

where the sum is taken over all $b=m+n i$, with $1 \leq m \leq k$ and $-k \leq n \leq k$. Then for all $\omega$

$$
R(\omega, t) \leq g(k, t)=M(k, t)+2^{t}\left(\frac{k+1}{k}\right)^{t} \frac{1}{k^{2 t-2}}\left(\frac{2 t+1}{(2 t-1)^{2} k}+\frac{1}{2 t-2}\right)
$$

the second term being an upper estimate on the remainder of the series $R(\omega, t)$. Using some estimates for numerical approximation and especially some programs written by Barbara Neuberger (which can be obtained through us), we find that $M(70,1.9)<0.99$ so that $g(70,1.9)<1$ and $\operatorname{HD}(J)<1.9$. We also note that this method fails at 1.88 since $M(50,1.88)>1$. Further studies with this method show that $\operatorname{HD}(J)<1.885$. To obtain sharper bounds on $\operatorname{HD}(J)$, improvements will be needed in our estimation methods, the programs and computing power.

Remark 6.7. Observe that proving estimates on $\left\|\phi_{\omega}^{\prime}\right\|$ and inf $\left|\phi_{\omega}^{\prime}\right|$ we have simultaneously provided techniques to find the best possible distortion constant $K$. Indeed, with the notation of the proof of Theorem 6.6 it was shown in [11], that $|1 / w-1 / 2| \leq 1 / 2$, where $w=q_{n} / q_{n-1}$. Thus $\operatorname{Re} w \geq 1$ and

$$
\begin{aligned}
K_{\omega} & =\sup \left\{\frac{\left|\phi_{\omega}^{\prime}(x)\right|}{\left|\phi_{\omega}^{\prime}(y)\right|}:|x-1 / 2|,|y-1 / 2| \leq 1 / 2\right\} \\
& =\sup \left\{\frac{|x+w|^{2}}{|y+w|^{2}}:|x-1 / 2|,|y-1 / 2| \leq 1 / 2\right\} .
\end{aligned}
$$


Therefore $K_{\omega}=|\beta(w+1 / 2)|^{2} /|\alpha(w+1 / 2)|^{2}=\beta^{2} / \alpha^{2}$. Rotate the line $\overline{0, w+1 / 2}$ to the positive $x$-axis with $w+1 / 2 \mapsto(x, 0), \alpha(w+1 / 2) \mapsto(x-1 / 2,0)$, and $\beta(w+1 / 2) \mapsto$ $(x+1 / 2,0)$. Note that $x \geq 3 / 2$. So, $\beta^{2} / \alpha^{2}=(x+1 / 2)^{2} /(x-1 / 2)^{2}$ and the maximum value occurs when $x=3 / 2$. Thus $K=\sup K_{\omega}=4$.

\section{Problems}

In this section we provide the reader with several problems.

Problem 7.1. What is the Hausdorff dimension $\operatorname{HD}(J)$ of the limit set $J$ of complex continued fractions described in Section 6? We feel that there is a good possibility that $\mathrm{HD}(J)$ will turn out to be some well-known number.

Problem 7.2. Extending the moduli of derivatives of all contractions $\phi_{b}(z)=\frac{1}{b+z}$, $\{m+n i:(m, n) \in \mathbb{N} \times \boldsymbol{Z}\}$, introduced at the very begining of the Section 6 , to uniformly bounded holomorphic functions defined on an open neighbourhood of $\overline{B(1 / 2,1 / 2)}$ in $\mathbb{C}^{2}$ and applying the Montel theorem we conclude that the Radon-Nikodym derivative $d m^{*} / d m$, the Gauss measure for complex continued fractions, is a real analytic function on $\overline{B(1 / 2.1 / 2)}$. What is its precise form?

Problem 7.3. Is it always true that if $m$ is the conformal measure, then $\operatorname{HD}(m)=$ $\operatorname{HD}(J)$ ?

Problem 7.4. Let $S=\left\{\phi_{i}: X \rightarrow X: i \in I\right\}$ be a regular conformal i.f.s.. For every Borel set $A \in J$ put $\sigma^{-1}(A)=\bigcup_{i \in I} \phi_{i}(A)$. We call a Borel probability measure $\nu$ on $J$ shift-invariant if $\nu\left(\sigma^{-1}(A)=\nu(A)\right.$ for all Borel subsets $A$ of $J$. We call it ergodic if all the sets satisfying $\sigma^{-1}(A)=A$ ) have either $\nu$ measure 0 or 1 . Is it true that $m^{*}$ is the only shift-invariant ergodic measure with $\operatorname{HD}\left(m^{*}\right)=\mathrm{HD}(J)$ ?

Problem 7.5. If the open set condition for a c.i.f.s. $S$ holds, then does the strong open set condition hold? At least, can one prove the results proven with the assumption that SOSC holds (Lemma 4.3 for example) assuming only OSC?

Problem 7.6. Does there exist a regular c.i.f.s. $S$ such that either measure $\mathrm{H}_{g}(J)$ or $\Pi_{g}(J)$ is either zero or infinity for every gauge function $g$ of the form $t^{h} L(t)$, where $L(t)$ is a slowly varying function?

Problem 7.7. Can one drop in Theorem 4.16 the assumption that $L(t)$ is a slowly varying function? 


\section{Appendix}

In this section we provide the reader with some results which are slightly aside of our flow of exposition and as well we outline an alternative approach for some topics contained in Section 3.

We call a Borel probability measure $\nu$ on $J$ invariant if $\sum_{i \in I} \nu \circ \phi_{i}=\nu$. From this point on, we assume $m$ is a $\delta$-conformal measure. We indicate a direct derivation of the measure $m^{*}$. This method makes no use of the abstract symbol space and is constructive-no use is made of Banach limits as in Section 3.

Lemma a.1. If there exists a Borel measurable function $\rho: J \rightarrow(0, \infty)$ such that $\mathcal{L} \rho=\rho$ and $\int \rho d \mu=1$, then the probability measure $\nu=\rho m$ is invariant and equivalent to $m$, whence equal to $m^{*}$.

Proof. The equivalency of $\nu$ and $m$ is obvious. In order to prove invariancy of $\nu$ notice that if $A \subset J$ is a Borel set, then

$$
\begin{aligned}
\sum_{i \in I} \nu \circ \phi_{i}(A) & =\sum_{i \in I} \int_{\phi_{i}(A)} \rho d m=\sum_{i \in I} \int_{A} \rho \circ \phi_{i}\left|\phi_{i}^{\prime}\right|^{\delta} d m=\int_{A} \sum_{i \in I} \rho \circ \phi_{i}\left|\phi_{i}^{\prime}\right|^{\delta} d m \\
& =\int_{A} \mathcal{L} \rho d m=\int_{A} \rho d m=\nu(A)
\end{aligned}
$$

The proof is finished.

Remark a.2. Employing methods similar to those of Section 3 such a measure $\nu$ can be proven to be ergodic and unique, but again no use of the shift representation of $J$ is required.

Lemma a.3. For all $x \in X$, let $\bar{\rho}(x)=\varlimsup_{n \rightarrow \infty} \mathcal{L}^{n} 1(x)$ and $\underline{\rho}(x)=\underline{\lim }_{n \rightarrow \infty} \mathcal{L}^{n} 1(x)$. Then $K^{-\delta} \leq \underline{\rho}(x) \leq \bar{\rho}(x) \leq K^{\delta}, \mathcal{L} \underline{\rho}(x) \leq \underline{\rho}(x)$ and $\mathcal{L} \bar{\rho}(x) \geq \bar{\rho}(x)$, for every $x \in X$.

Proof. Inequalities $K^{-\delta} \leq \underline{\rho}(x) \leq \bar{\rho}(x) \leq K^{\delta}$ are obvious. In order to prove that $\mathcal{L} \bar{\rho}(x) \geq \bar{\rho}(x)$, fix $\varepsilon>0$ and take a finite subset $I_{1}$ of $I$ such that $\sum_{i \in I_{2}}\left\|\phi_{i}^{\prime}\right\|^{\delta}<\varepsilon$, where $I_{2}=I \backslash I_{1}$. Since $I_{1}$ is finite, there exists $k \geq 1$ such that $\bar{\rho}\left(\phi_{i}(x)\right) \geq \mathcal{L}^{n} 1\left(\phi_{i}(x)\right)-\varepsilon$ for all $i \in I_{1}$ and all $n \geq k$. By the definition of $\bar{\rho}(x)$, there exists $q \geq k$ such that 
$\mathcal{L}^{q+1} 1(x) \geq \bar{\rho}(x)-\varepsilon$. Therefore, we get

$$
\begin{aligned}
\mathcal{L} \bar{\rho}(x) & =\sum_{i \in I_{1}} \bar{\rho}\left(\phi_{i}(x)\right)\left|\phi_{i}^{\prime}(x)\right|^{\delta}+\sum_{i \in I_{2}} \bar{\rho}\left(\phi_{i}(x)\right)\left|\phi_{i}^{\prime}(x)\right|^{\delta} \\
& \geq \sum_{i \in I_{1}} \bar{\rho}\left(\phi_{i}(x)\right)\left|\phi_{i}^{\prime}(x)\right|^{\delta} \geq \sum_{i \in I_{1}}\left(\mathcal{L}^{q} 1\left(\phi_{i}(x)\right)-\varepsilon\right)\left|\phi_{i}^{\prime}(x)\right|^{\delta} \\
& =\sum_{i \in I_{1}} \mathcal{L}^{q} 1\left(\phi_{i}(x)\right)\left|\phi_{i}^{\prime}(x)\right|^{\delta}-\varepsilon \sum_{i \in I_{1}}\left|\phi_{i}^{\prime}(x)\right|^{\delta} \\
& =\mathcal{L}^{q+1} 1(x)-\sum_{i \in I_{2}} \mathcal{L}^{q} 1\left(\phi_{i}(x)\right)\left|\phi_{i}^{\prime}(x)\right|^{\delta}-\varepsilon \sum_{i \in I_{1}}\left|\phi_{i}^{\prime}(x)\right|^{\delta} \\
& \geq \mathcal{L}^{q+1} 1(x)-K^{\delta} \varepsilon-K^{\delta} \varepsilon \geq \bar{\rho}(x)-\varepsilon-2 K^{\delta} \varepsilon \\
& =\bar{\rho}(x)-\left(1+2 K^{\delta}\right) \varepsilon .
\end{aligned}
$$

So, letting $\varepsilon \rightarrow 0$ we get $\mathcal{L} \bar{\rho}(x) \geq \bar{\rho}(x)$. The proof that $\mathcal{L} \underline{\rho}(x) \leq \underline{\rho}(x)$ is similar.

Lemma a.4. For every $x \in X$, the limits $\bar{\rho}_{\infty}(x)=\lim _{n \rightarrow \infty} \mathcal{L}^{n} \bar{\rho}(x)$ and $\underline{\rho}_{\infty}(x)=$ $\lim _{n \rightarrow \infty} \mathcal{L}^{n} \underline{\rho}(x)$ exist. Moreover $\mathcal{L} \bar{\rho}_{\infty}(x)=\bar{\rho}_{\infty}(x)$ and $\mathcal{L} \underline{\rho}_{\infty}(x)=\underline{\rho}_{\infty}(x)$ for all $x \in X$.

Proof. Since $\mathcal{L}$ is a positive operator, it is monotone, and therefore, it follows from Lemma a.3 that the sequence $\left\{\mathcal{L}^{n} \underline{\rho}(x): n \geq 0\right\}$ is non-increasing and bounded from below by $K^{-2 \delta}$. Thus, it is convergent and denote its limit by $\underline{\rho}_{\infty}(x)$. Since $\underline{\rho}_{\infty} \leq \mathcal{L}^{n} \underline{\rho}$ for every $n \geq 1$, we get $\mathcal{L} \underline{\rho}_{\infty} \leq \mathcal{L}^{n+1} \underline{\rho}$ and therefore $\mathcal{L} \underline{\rho}_{\infty} \leq \underline{\rho}_{\infty}$. In order to prove that $\overline{\mathcal{L}}_{\infty} \geq \underline{\rho}_{\infty}$ fix $x \in X$ and $\varepsilon>0$. Take a finite subset $I_{1}$ of $I$ such that $\sum_{i \in I_{2}}\left\|\phi_{i}^{\prime}\right\|^{\delta}<\varepsilon$, where $I_{2}=I \backslash I_{1}$. Since $I_{1}$ is finite and since all sequences $\left\{\mathcal{L}^{n} \rho(z): n \geq 0\right\}, z \in X$, converge, there is $n \geq 0$ such that $\underline{\rho}_{\infty}\left(\phi_{i}(x)\right) \geq \mathcal{L}^{n} \underline{\rho}\left(\phi_{i}(x)\right)-\varepsilon$, for all $i \in I_{1}$. We then have

$$
\begin{aligned}
\mathcal{L} \underline{\rho}_{\infty}(x) & \geq \sum_{i \in I_{1}} \underline{\rho}_{\infty}\left(\phi_{i}(x)\right)\left|\phi_{i}^{\prime}(x)\right|^{\delta} \geq \sum_{i \in I_{1}}\left(\mathcal{L}^{n} \underline{\rho}\left(\phi_{i}(x)\right)-\varepsilon\right)\left|\phi_{i}^{\prime}(x)\right|^{\delta} \\
& \geq \sum_{i \in I_{1}} \mathcal{L}^{n} \underline{\rho}\left(\phi_{i}(x)\right)\left|\phi_{i}^{\prime}(x)\right|^{\delta}-K^{\delta} \varepsilon=\mathcal{L}^{n+1} \underline{\rho}(x)-\sum_{i \in I_{2}} \mathcal{L}^{n} \underline{\rho}\left(\phi_{i}(x)\right)\left|\phi_{i}^{\prime}(x)\right|^{\delta}-K^{\delta} \varepsilon \\
& \geq \underline{\rho}_{\infty}(x)-K^{\delta} \varepsilon-K^{\delta} \varepsilon=\underline{\rho}_{\infty}(x)-2 K^{\delta} \varepsilon
\end{aligned}
$$

So, letting $\varepsilon \rightarrow 0$ we get the required inequality. The case of the function $\bar{\rho}_{\infty}$ can be dealt with similarly.

The following corollary is an immediate consequence of Lemma a.4, Lemma a.1 and Remark a.2 following it or Theorem 3.8.

Corollary a.5. For $m$-a.e. $x \in J$, we have $\underline{\rho}_{\infty}(x)=\underline{\rho}(x)$ and $\bar{\rho}_{\infty}(x)=\bar{\rho}(x)$.

Next, we obtain a sufficient condition for the measure $\nu$ to have a continuous RadonNikodym derivative with respect to $m$.

Lemma a.6. Fix a compact set $X \subset Y \subset V$. If there exists $q \geq 1$ such that the family of functions $\left\{\log \mid \phi_{\omega}^{\prime} \|_{\phi_{\tau}(Y)}: \tau \in I^{q}, \omega \in I^{\infty}\right\}$ is equicontinuous, then for every continuous 
function $f: Y \rightarrow \mathbb{R}$, the family $\left\{\mathcal{L}^{n}(f): Y \rightarrow \mathbb{R}: n \geq 1\right\}$ is equicontinuous, i.e. the Perron-Frobenius operator $\mathcal{L}: C(Y) \rightarrow C(Y)$ is almost periodic. In this case, there is a continuous function $\rho: J \rightarrow(0, \infty)$ such that $\mathcal{L} \rho=\rho$ and $\int \rho d \mu=1$.

Proof. First note that in view of (3.3) there exists a finite set $I_{1}^{q} \subset I^{q}$ such that writing $I_{2}^{q}=I^{q} \backslash I_{1}^{q}$ we have $\sum_{\gamma \in I_{2}^{q}}\left\|\phi_{\gamma}^{\prime}\right\|^{\delta}<\varepsilon$. Now, it is of course enough to prove that the family $\left\{\mathcal{L}^{n}(f): Y \rightarrow \mathbb{R}: n \geq q\right\}$ is equicontinuous. So, fix $\varepsilon>0$. Take $\gamma>0$ so small that $|f(y)-f(x)|<\varepsilon$ and $\left.\sum_{\gamma \in I_{1}^{q}}|| \phi_{\gamma}^{\prime}(y)\right|^{\delta}-\left|\phi_{\gamma}^{\prime}(x)\right|^{\delta} \mid<\varepsilon$ if $|y-x|<\gamma, x, y \in Y$, and moreover $\left|\log \left(\left|\phi_{\omega}^{\prime}(y)\right|\right)-\log \left(\left|\phi_{\omega}^{\prime}(x)\right|\right)\right|<\varepsilon$ if $|y-x|<\gamma, x, y \in Y$, and $|\omega| \geq q$.

Take now $x, y \in Y$ with $|y-x|<\eta$. Then by (3.3)

$$
\begin{aligned}
\mid \mathcal{L}^{n} f(y) & -\mathcal{L}^{n} f(x) \mid= \\
& =\sum_{\omega \in I^{n}}\left(\left|\phi_{\omega}^{\prime}(y)\right|^{\delta} f\left(\phi_{\omega}(y)\right)-\left|\phi_{\omega}^{\prime}(x)\right|^{\delta} f\left(\phi_{\omega}(x)\right)\right) \\
& \leq \sum_{\omega \in I^{n}}\left|\phi_{\omega}^{\prime}(y)\right|^{\delta}\left|f\left(\phi_{\omega}(y)\right)-f\left(\phi_{\omega}(x)\right)\right|+\left.\sum_{\omega \in I^{n}}\left|f\left(\phi_{\omega}(x)\right)\right||| \phi_{\omega}^{\prime}(y)\right|^{\delta}-\left|\phi_{\omega}^{\prime}(x)\right|^{\delta} \mid \\
& \leq K^{\delta} \varepsilon+|| f \|\left._{\infty} \sum_{\omega \in I^{n}}|| \phi_{\omega}^{\prime}(y)\right|^{\delta}-\left|\phi_{\omega}^{\prime}(x)\right|^{\delta} \mid
\end{aligned}
$$

In order to estimate the last summand in the display above observe that

$$
\begin{aligned}
\left.\sum_{\omega \in I^{n}}|| \phi_{\omega}^{\prime}(y)\right|^{\delta} & -\left|\phi_{\omega}^{\prime}(x)\right|^{\delta} \mid= \\
& =\sum_{\tau \in I^{n-q}} \sum_{\eta \in I^{q}}\left(\left.|| \phi_{\tau}^{\prime}\left(\phi_{\eta}(y)\right)\right|^{\delta}\left|\phi_{\eta}^{\prime}(y)\right|^{\delta}-\left|\phi_{\tau}^{\prime}\left(\phi_{\eta}(x)\right)\right|^{\delta}\left|\phi_{\eta}^{\prime}(x)\right|^{\delta} \mid\right) \\
& \leq\left.\sum_{\tau \in I^{n q}} \sum_{\eta \in I^{q}}\left(\left.\left|\phi_{\tau}^{\prime}\left(\phi_{\eta}(y)\right)\right|^{\delta}|| \phi_{\eta}^{\prime}(y)\right|^{\delta}-\mid \phi_{\eta}^{\prime}(x)\right)\right|^{\delta} \mid+ \\
& \leq \sum_{\tau \in I^{n-q}} \sum_{\eta \in I^{q}}\left|\phi_{\tau}^{\prime}\left(\phi_{\eta}(y)\right)\right|^{\delta}\left(\left.|| \phi_{\eta}^{\prime}(y)\right|^{\delta}-\left|\phi_{\eta}^{\prime}(x)\right|^{\delta} \mid+\right. \\
& \leq \sum_{\tau \in I^{n-q}} \sum_{\eta \in I^{q}}\left(\left|\phi_{\tau}^{\prime}\left(\phi_{\eta}(y)\right)\right|^{\delta}||\left|\phi_{\eta}^{\prime}(y)\right|^{\delta}-\left|\phi_{\eta}^{\prime}(x)\right|^{\delta} \mid\right)+\delta K^{2 \delta} \varepsilon
\end{aligned}
$$

Let us estimate the first summand in the last line of this display. We have

$$
\begin{gathered}
\left.\sum_{\tau \in I^{n-q}} \sum_{\eta \in I_{2}^{q}} \mid \phi_{\tau}^{\prime}\left(\phi_{\eta}(y)\right)\right)\left.\right|^{\delta}\left|\| \phi_{\eta}^{\prime}(y)\right|^{\delta}-\left|\phi_{\eta}^{\prime}(x)\right|^{\delta} \mid \\
\leq\left.\sum_{\tau \in I^{n-q}} \sum_{\eta \in I_{2}^{q}}\left\|\phi_{\tau}^{\prime}\right\|\right|^{\delta} 2\left\|\phi_{\eta}^{\prime}\right\|^{\delta} \\
\leq 2 K^{\delta} \varepsilon
\end{gathered}
$$


and

$$
\begin{aligned}
\left.\sum_{\tau \in I^{n-q}} \sum_{\eta \in I_{1}^{q}} \mid \phi_{\tau}^{\prime}\left(\phi_{\eta}(y)\right)\right)\left.\right|^{\delta}||\left|\phi_{\eta}^{\prime}(y)\right|^{\delta}-\left|\phi_{\eta}^{\prime}(x)\right|^{\delta} \mid \\
\quad \leq\left.\left.\sum_{\tau \in I^{n-q}}|| \phi_{\tau}^{\prime}\right|^{\delta} \sum_{\eta \in I_{1}^{q}}|| \phi_{\eta}^{\prime}(y)\right|^{\delta}-\left|\phi_{\eta}^{\prime}(x)\right|^{\delta} \mid \\
\leq K^{\delta} \varepsilon
\end{aligned}
$$

Thus combining (a.1)-(a.4) we get $\left|\mathcal{L}^{n} f(y)-\mathcal{L}^{n} f(x)\right| \leq K^{\delta}\left(1+\|f\|_{\infty}\left(3+\delta K^{\delta}\right)\right) \varepsilon$ which finishes the proof.

Remark a.7. We would like to remark that if the family $\left\{\mathcal{L}^{n}(f): X \rightarrow \mathbb{R}: n \geq 1\right\}$ is equicontinuous, in particular if the assumptions of condition (c) of Lemma 2.2, corresponding to condition (iii), p.125, in [38], are satisfied, or even weaker if the assumptions of Lemma a.6 are satisfied, then using similar arguments as in the proof of Theorem 8 of [38], one can show that there exists a unique continuous function $g: X \rightarrow(0, \infty)$ such that $\mathcal{L}(g)=g$ on $X$. Suppose that $g$ is normalized so that $\int g d m=1$. Then $g$ restricted to the limit set $J$ is a version of the Radon-Nikodym derivative $d m^{*} / d m$. Moreover for every continuous function $f: X \rightarrow \mathbb{R}$,

$$
\mathcal{L}^{n}(f) \longrightarrow g \int f d m
$$

uniformly on $X$. Also some additional ergodic properties of the system $\left(\sigma, m^{*}\right)$, for example the weak Bernoulli property, can be proven as in [38].

Acknowledgements. We would like to express our gratitude to Barbara Neuberger for her numerical assistance in completing the proof of Theorem 6.6. The second author wishes to thank the SFB 170 at the Institute of Mathematics in Goettingen for its warm hospitality and financial support during the preparation of this paper. 


\section{REFERENCES}

[1] J. Aronson, M. Denker, M. Urbański, Ergodic theory for Markov fibered systems and parabolic rational maps, Transactions of A.M.S. 337 (1993), 495-548

[2] T. Bedford, Hausdorff dimension and box dimension in self-similar sets, Proc. Topology and Measure V, Ernst-Moritz-Arndt Universität Greisfwald (1988).

[3] R. Bowen: Hausdorff dimension of quasi-circles. Publ. IHES 50 (1980), 11-25.

[4] M. Denker, M. Urbański, Hausdorff and conformal measures on Julia sets with a rationally indifferent periodic point, J. London Math. Soc. 43 (1991), 107 - 118.

[5] M. Denker, M. Urbański, On absolutely continuous invariant measures for expansive rational maps with rationally indifferent periodic points, Forum Math. 3(1991), 561 - 579

[6] M. Denker, M. Urbański, Geometric measures for parabolic rational maps, Ergod. Th. \& Dynam. Sys. 12 (1992), 53 - 66.

[7] M. Denker, M. Urbański, The capacity of parabolic Julia sets, Math. Zeitsch. 211 (1992), 73 - 86 .

[8] K. J. Falconer, The geometry of fractal sets, Cambridge Univ. Press, Cambridge, 1985.

[9] K.J. Falconer, Fractal geometry, J. Wiley, New York, 1990.

[10] H.F. Federer, Geometric measure theory, Springer Verlag, New York Inc. 1969.

[11] R.J. Gardner, R.D. Mauldin, On the Hausdorff dimension of a set of complex continued fractions, Illinois J. of Math. 27 (1983), 334 - 344.

[12] S. Graf, R.D. Mauldin, S.C. Williams, The exact Hausdorff dimension in random recursive constructions, Memoirs of A.M.S. 381 (1988).

[13] M. Guzmán, Differentiation of integrals in $\mathbb{R}^{n}$. Lect. Notes in Math. 481, Springer Verlag, New York.

[14] J. E. Hutchinson, Fractals and self-similarity, Indiana Univ. Math. J. 30 (1981), 713 $-747$.

[15] A. Manning, H. McCluskey: Hausdorff dimension for horseshoes. Ergod. Th. and Dynam. Sys. 3 (1983), 251-260.

[16] P. Mattila, Geometry of sets and measures in euclidean spaces, Cambridge University Press, to appear.

[17] R.D. Mauldin, S.C. Williams, Random recursive constructions: Asymptotic geometric and topological properties, Transactions of A.M.S. 259 (1986), 325 - 346.

[18] P.A.P. Moran, Additive functions of intervals and Hausdorff measure, Proc. Cambridge, Philos. Soc. 42 (1946), 15 - 23.

[19] P. J. Nicholls, The ergodic theory of discrete groups, LMS Lect. Notes Series 143, Cambridge University Press, 1989.

[20] W. Parry, Entropy and generators in ergodic theory, W. A. Benjamin, 1969.

[21] S. J. Patterson, The limit set of a Fuchsian group, Acta Math. 136, (1976), 241-273. 
[22] S. J. Patterson, Lectures on measures on limit sets of Kleinian groups, in "Analytical and geometric aspects of hyperbolic space, LMS Lect. Notes Series 111, Cambridge University Press, 1989.

[23] Ch. Pommerenke, Boundary behaviour of conformal maps, Springer-Verlag, Berlin, Heidelberg, 1992.

[24] A. Renyi, Representations for real numbers and and their ergodic properties, Acta Mat. Acad. Sci. Hung. 8 (1957), 477-493.

[25] C. Rogers, S.J. Taylor, Functions continuous and singular with respect to a Hausdorff measure, Mathematika, 8 (1961), 1-31.

[26] D. Ruelle, Thermodynamic formalism, Addison Wesley, 1978.

[27] D. Ruelle, An extension of the theory of Fredholm determinants, Publ. Math. I.H.E.S. 72 (1990), 173-193.

[28] A. Schief, Separation properties of self-similar sets, Proc. Amer. Math. Soc., 122 (1994), 111-115.

[29] F. Schweiger, Number theoretical endomorphisms with $\sigma$-finite invariant measures. Isr. J. Math. 21, (1975), 308-318.

[30] F. Schweiger, Ergodic properties of fibred systems, University of Salzburg preprint 1989.

[31] B. Strattmann, M. Urbański, The box-counting dimension for geometrically finite Kleinian groups, University of Göttingen preprint 1993.

[32] D. Sullivan, The density at infinity of a discrete group of hyperbolic motions, Publ. IHES, 50 (1979), 171-121.

[33] D. Sullivan, Conformal dynamical systems. In: Geometric Dynamics. Lect. Notes in Math. 1007 (1983), 725 - 752, Springer Verlag.

[34] D. Sullivan, Entropy, Hausdorff measures old and new, and limit sets of geometrically Kleinian groups, Acta Math. 153 (1984), 259 - 277.

[35] S.J. Taylor, C. Tricot, Packing measure, and its evaluation for a Brownian path, Trans. A.M.S. 288 (1985), 679 - 699.

[36] M. Urbański, Rational functions with no recurrent critical points, Ergod. Th. \& Dynam. Sys. 14 (1994), 391-414.

[37] P. Walters, A variational principle for the pressure of continuous transformations, Amer. J. Math. 97 (1975), 937 - 971.

[38] P. Walters, Invariant measures and equilibrium states for some mappings which expand distances, Transactions of A.M.S. 236 (1978), 121 - 153.

[39] P. Walters, An introduction to ergodic theory, Springer-Verlag, 1982. 